\title{
PROBABILISTIC INTERPRETATION AND RANDOM WALK ON SPHERES ALGORITHMS FOR THE POISSON-BOLTZMANN EQUATION IN MOLECULAR DYNAMICS
}

\author{
Mireille Bossy $^{1}$, Nicolas Champagnat $^{1}$, Sylvain Maire $^{2}$ and Denis Talay ${ }^{1}$
}

\begin{abstract}
Motivated by the development of efficient Monte Carlo methods for PDE models in molecular dynamics, we establish a new probabilistic interpretation of a family of divergence form operators with discontinuous coefficients at the interface of two open subsets of $\mathbb{R}^{d}$. This family of operators includes the case of the linearized Poisson-Boltzmann equation used to compute the electrostatic free energy of a molecule. More precisely, we explicitly construct a Markov process whose infinitesimal generator belongs to this family, as the solution of a SDE including a non standard local time term related to the interface of discontinuity. We then prove an extended Feynman-Kac formula for the Poisson-Boltzmann equation. This formula allows us to justify various probabilistic numerical methods to approximate the free energy of a molecule. We analyse the convergence rate of these simulation procedures and numerically compare them on idealized molecules models.
\end{abstract}

Mathematics Subject Classification. 35Q60, 92C40, 60J60, 65C05, 65C20, 68U20.

Received July 31st, 2009. Revised February 23, 2010.

Published online August 26, 2010.

\section{Introduction}

This paper deals with two closely related questions. First, we establish a novel probabilistic interpretation of divergence-form operators in $\mathbb{R}^{d}, d \geq 1$, of the form

$$
\mathcal{L}=\nabla \cdot(\varepsilon(x) \nabla),
$$

where $\varepsilon$ is a piecewise constant function from $\mathbb{R}^{d}$ to $(0,+\infty)$ with a smooth discontinuity manifold $\Gamma$. Second, we derive from this probabilistic interpretation numerical Monte Carlo methods for the linearized PoissonBoltzmann equation in molecular dynamics [2,3] (which is actually a Poisson equation in the classical PDE terminology):

$$
-\nabla \cdot(\varepsilon(x) \nabla u(x))+\kappa^{2}(x) u(x)=f(x), \quad x \in \mathbb{R}^{3},
$$

\footnotetext{
Keywords and phrases. Divergence form operator, Poisson-Boltzmann equation, Feynman-Kac formula, random walk on sphere algorithm.

1 TOSCA project-team, INRIA Sophia Antipolis - Méditerranée, France. Mireille.Bossy@sophia.inria.fr;

Nicolas.Champagnat@sophia.inria.fr; Denis.Talay@sophia.inria.fr

2 IMATH, Université du sud Toulon-Var, France. maire@univ-tln.fr
} 
where

$$
\varepsilon(x)=\left\{\begin{array}{lll}
\varepsilon_{\mathrm{int}}>0 & \text { if } x \in \Omega_{\mathrm{int}}, \\
\varepsilon_{\mathrm{ext}}>0 & \text { if } x \in \Omega_{\mathrm{ext}},
\end{array} \quad \kappa(x)= \begin{cases}0 & \text { if } x \in \Omega_{\mathrm{int}}, \\
\bar{\kappa}>0 & \text { if } x \in \Omega_{\mathrm{ext}},\end{cases}\right.
$$

$\Omega_{\text {int }}$ and $\Omega_{\text {ext }}$ being two open subsets of $\mathbb{R}^{3} ; \Omega_{\text {int }}$ is bounded with boundary $\Gamma, \Omega_{\text {int }} \cap \Omega_{\text {ext }}=\emptyset$, and $\bar{\Omega}_{\text {int }} \cup \bar{\Omega}_{\text {ext }}=\mathbb{R}^{3}$ (see Fig. 1). We also define $\varepsilon(x)=\varepsilon_{\text {int }}$ and $\kappa(x)=0$ for all $x \in \Gamma$, although the precise value of these functions on $\Gamma$ has no influence on the results of this paper. The source term is

$$
f:=\sum_{i=1}^{N} q_{i} \delta_{x_{i}}
$$

where $\delta_{x}$ denotes the Dirac unit measure at $x \in \mathbb{R}^{3}, x_{i} \in \Omega_{\text {int }}$ and $q_{i} \in \mathbb{R}$ for all $i \in\{1, \ldots, N\}$. This PoissonBoltzmann equation describes the electrostatic potential around a biomolecular assembly, composed of $N$ atoms at position $x_{i}$ and with charges $q_{i}$. The set $\Omega_{\text {int }}$ is the interior of the molecule, that is, the union of finitely many spheres representing the atoms of the molecules. The set $\Omega_{\mathrm{ext}}$ is the exterior of the molecule.

It is well known that general divergence form operators define symmetric Dirichlet forms. The connection between symmetric Dirichlet forms and Dirichlet Markov processes is well developed [8]. In general, such processes can be decomposed into a local martingale and a zero-quadratic variation part with possibly infinite variation. They can also be decomposed into the sum of two processes which are semimartingales w.r.t. two different filtrations (see, e.g., [30]). Both decompositions involve processes which are only implicitly defined, which makes impossible to derive from them Monte Carlo approximations of $u(x)$ (discretization schemes for general Dirichlet processes have recently been developed in [18] but their numerical efficiency is questionable).

In the one dimensional case $(d=1)$, one can develop other probabilistic interpretations which are suitable to Monte Carlo simulations, for example in terms of stochastic differential equations (SDEs) involving the local time of the solution: in the case where $\varepsilon(x)=\varepsilon_{\text {int }}$ if $x<0$ and $\varepsilon(x)=\varepsilon_{\text {ext }}$ if $x \geq 0$, such a SDE writes

$$
\mathrm{d} X_{t}=\sqrt{2 \varepsilon\left(X_{t}\right)} \mathrm{d} B_{t}+\frac{\varepsilon_{\text {ext }}-\varepsilon_{\text {int }}}{2 \varepsilon_{\text {ext }}} \mathrm{d} L_{t}^{0}(X),
$$

where $L_{t}^{0}(X)$ stands for the local time at point 0 of the continuous semimartingale $X$ (see e.g. Protter [26], Sect. IV.7, for a definition). For existence and uniqueness of a solution to this SDE, see, e.g., Portenko [24,25] and Le Gall [14]. Numerical approximations of the process $\left(X_{t}\right)$ have been developed and analyzed by Lejay [15], Lejay and Martinez [17], Martinez [21], Martinez and Talay [22].

In the multidimensional case $(d>1)$, the situation is not so well understood. Our goal here is to construct a Markov process whose generator is $\mathcal{L}$ and which can easily be simulated on a computer. We take advantage of the particular structure of $\varepsilon(x)$.

The paper is organized as follows. Section 2 is devoted to the probabilistic interpretation of the operator (1.1). After giving some notation (Sect. 2.1), we define the adequate martingale problem for the operator $\mathcal{L}$ in Section 2.2. In Section 2.3, the SDE corresponding to the operator $\mathcal{L}$ is defined and a weak solution $X$ is constructed. Next, we extend Itô's formula to the process to $X$ and to functions having a discontinuous normal gradient on $\Gamma$ in Section 2.4. The relationship between $X$ and solutions of the martingale problem of Section 2.2 is then established in Section 2.5. Next, well-posedness of the martingale problem and the strong Markov property for $X$ are proved in Section 2.6. Finally, in Section 2.7, Feynman-Kac's formula is extended to the solution of PDEs involving the operator (1.1). Section 3 is devoted to the probabilistic interpretation of Poisson-Boltzmann equation (Sect. 3.1), and to the description of Monte Carlo resolution algorithms, based on various discretizations of the process $X$, which extend the algorithm proposed by Mascagni and Simonov [23] (Sect. 3.2). These algorithms, described in Section 3.3, are based on walk on spheres techniques inside and outside the molecule, and either on explicit computations extending the ones of [23], or on neutron transport type approximations when the particle lies on the set of discontinuity $\Gamma$. Section 4 is devoted to the error analysis of the algorithms, first in the case where $\Omega_{\text {int }}$ is a single sphere (Sect. 4.1), and next in the general case 


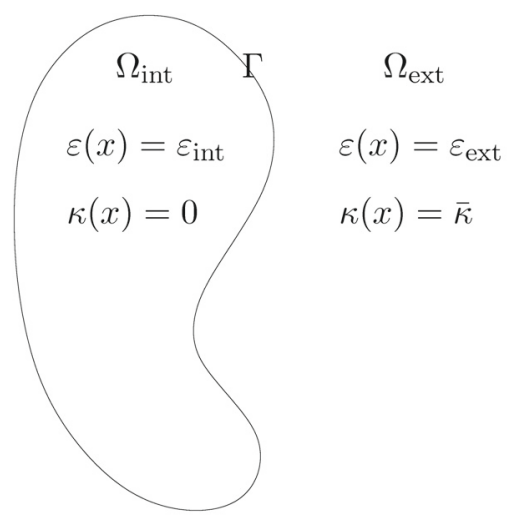

FiguRE 1. Definition of $\Omega_{\text {int }}, \Omega_{\text {ext }}, \Gamma, \varepsilon(x)$ and $\kappa(x)$.

(Sect. 4.2). Finally, Section 5 presents numerical results in the case of one and two atoms. In particular, we show that our new methods improve significantly the methods in [23], and that the neutron transport method offers a better trade-off between error and computational time than the other methods.

\subsection{Notation}

Throughout this paper, we use the following functional spaces.

- For all open subset of $\mathbb{R}^{d}$, the set $C^{k}(D)$ (resp. $C_{b}^{k}(D)$, resp. $C_{c}^{k}(D)$ ) is the set of $k$ times continuously differentiable functions on $D$ (respectively with bounded derivatives of all order between 0 and $k$, or with compact support in $D$ ).

- If the set $D$ has a $C^{1}$ boundary $\Gamma$ but is not open, the sets $C^{k}(D), C_{b}^{k}(D)$ and $C_{c}^{k}(D)$ are defined as the sets $C^{k}(\stackrel{\circ}{D}), C_{b}^{k}(\stackrel{\circ}{D})$ and $C_{c}^{k}(\stackrel{\circ}{D})$, respectively, with the additional property that derivatives of all order up to $k$ can be continuously extended to $\Gamma \cap D$.

- $C(D), C_{b}(D)$ and $C_{c}(D)$ denote the sets $C^{0}(D), C_{b}^{0}(D)$ and $C_{c}^{0}(D)$, respectively.

- For $p \in[1, \infty]$ and $k \geq 1$, the set $W^{k, p}(D)$ (resp. $\left.W_{\mathrm{loc}}^{k, p}(D)\right)$ is the set of functions admitting derivatives in the sense of the distributions in $L^{p}(D)$ (respectively $L_{\text {loc }}^{p}(D)$ ) up to order $k$.

- We respectively denote the sets $W^{k, 2}(D)$ and $W_{\text {loc }}^{k, 2}(D)$ by $H^{k}(D)$ and $H_{\mathrm{loc}}^{k}(D)$.

Moreover, for all differentiable function $\phi, J(\phi)$ denotes the Jacobian matrix of $\phi$, the standard Euclidean scalar product between $u, v \in \mathbb{R}^{d}$ is denoted by $u \cdot v$, and the Euclidean norm of $u$ by $\|u\|$.

\section{Probabilistic interpretation of Divergence Form operators WITH A PIECEWISE CONSTANT COEFFICIENT}

In this section we construct a stochastic Markov process whose infinitesimal generator is the divergence form operator (1.1), we extend several stochastic calculus tools to this process, and we connect this process to elliptic PDEs driven by the operator (1.1).

We assume that the dimension $d \geq 1$ is arbitrary. We also assume that $\Omega_{\text {int }}$ is a bounded open simply connected set with boundary $\Gamma:=\partial \Omega_{\text {int }}{ }^{3}$, and that $\Omega_{\text {ext }}=\mathbb{R}^{d} \backslash\left(\Omega_{\text {int }} \cup \Gamma\right)$. Unless explicitly mentioned, the compact manifold $\Gamma$ is assumed to be of class $C^{3}$.

\footnotetext{
${ }^{3}$ The assumption that $\Omega_{\text {int }}$ is simply connected can easily be relaxed. We restrict to the simply connected case for simplicity, and because this case is relevant in Molecular Dynamics.
} 


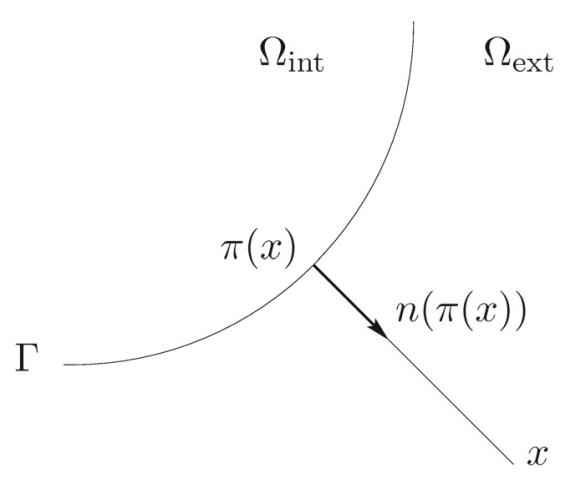

Figure 2. Definition of $\pi(x)$ and $n(\pi(x))$.

\subsection{A particular family of homeomorphisms in a neighbourhood of $\Gamma$}

Since the boundary $\Gamma$ is smooth, one can construct a mapping $\pi$ of class $C_{b}^{2}$ from a neighborhood $\mathcal{N}$ of $\Gamma$ to $\Gamma$ such that

$$
|x-\pi(x)|=\mathrm{d}(x, \Gamma), \quad \forall x \in \mathcal{N}
$$

where $\mathrm{d}(x, \Gamma)$ denotes the distance between $x$ and the set $\Gamma$. For all $x \in \Gamma$, let $n(x)$ denote the unit vector normal to $\Gamma$ at $x$ and pointing in the direction of $\Omega_{\text {ext }}$ (see Fig. 2). Finally, for all $x \in \mathcal{N}$, set

$$
\rho(x):=(x-\pi(x)) \cdot n(\pi(x))
$$

thus $\rho(x)$ is the signed distance to $\Gamma$ (positive in $\Omega_{\text {ext }}$, negative in $\Omega_{\text {int }}$ ) and is of class $C_{b}^{2}(\mathcal{N})$ (actually, it is even $C_{b}^{3}(\mathcal{N})$ by $(2.1)$ below). We still denote by $\rho$ a $C_{b}^{2}\left(\mathbb{R}^{d}\right)$ extension of this function to the whole Euclidean space. It is well-known that

$$
\nabla \rho(x)=n(\pi(x)), \quad \forall x \in \mathcal{N}
$$

(see e.g. [9], p. 355). Note that, reducing $\mathcal{N}$ if necessary, we can always assume that $\pi$ is $C_{b}^{2}(\overline{\mathcal{N}})$.

Our construction of a stochastic process admitting (1.1) as generator makes use of the following family local straightenings of $\Gamma$.

Proposition 2.1. There exists a family of bounded open subsets of $\mathcal{N},\left\{\mathcal{U}_{1}, \ldots, \mathcal{U}_{M-1}\right\}$ such that $\Gamma \subset \cup_{i=1}^{M-1} \mathcal{U}_{i}$, and a family of functions $\left\{\psi_{1}, \ldots, \psi_{M-1}\right\}$ such that, for all $1 \leq i \leq M-1, \psi_{i}=\left(\psi_{i}^{1}, \ldots, \psi_{i}^{d}\right)$ is a $C_{b}^{2}$ diffeomorphism from $\mathcal{U}_{i}$ to $\psi_{i}\left(\mathcal{U}_{i}\right)$, admitting a $C_{b}^{2}$ extension on $\overline{\mathcal{U}_{i}}$ and satisfying for all $x \in \overline{\mathcal{U}_{i}}$

$$
\begin{gathered}
\psi_{i}^{1}(x)=\rho(x), \\
\nabla \psi_{i}^{k}(x) \cdot n \circ \pi(x)=0, \quad \forall k \in\{2,3, \ldots, d\}, \\
\frac{\partial \psi_{i}^{-1}}{\partial x_{1}}\left(\psi_{i}(x)\right)=n \circ \pi(x) .
\end{gathered}
$$

Note that, by $(2.2), \psi_{i}\left(\mathcal{U}_{i} \cap \Gamma\right) \subset\{0\} \times \mathbb{R}^{d-1}$, which justifies the term "local straightening". Before proving this result, we introduce a modification of these functions used repeatedly in the sequel. Define the function $F: \mathbb{R}^{d} \rightarrow \mathbb{R}^{d}$ by

$$
F\left(x_{1}, \ldots, x_{d}\right):=\left(\frac{x_{1}}{\varepsilon^{\#}\left(x_{1}\right)}, x_{2}, \ldots, x_{d}\right), \quad \forall x \in \mathbb{R}^{d},
$$


where $\varepsilon^{\#}: \mathbb{R} \rightarrow \mathbb{R}$ is given by

$$
\varepsilon^{\#}(x):= \begin{cases}\varepsilon_{\text {int }} & \text { if } x \leq 0 \\ \varepsilon_{\text {ext }} & \text { if } x>0\end{cases}
$$

Then, for all $1 \leq i \leq d$, we define the homeomorphism

$$
\bar{\psi}_{i}(x)=F \circ \psi_{i}(x), \quad \forall x \in \mathcal{U}_{i}
$$

and $\mathcal{V}_{i}=\bar{\psi}_{i}\left(\mathcal{U}_{i}\right)$. Note that $\bar{\psi}_{i}^{1}$ is continuous but not $C^{1}$ on $\Gamma$, and $\bar{\psi}_{i}^{2}, \ldots, \bar{\psi}_{i}^{d}$ are $C^{1}$. Finally, let $\mathcal{U}_{M}=\mathcal{V}_{M}$ be an open subset of $\mathbb{R}^{d}$ such that $\Gamma \cap \mathcal{U}_{M}=\emptyset$ and $\cup_{i=1}^{M} \mathcal{U}_{i}=\mathbb{R}^{d}$, and set $\bar{\psi}_{M}(x):=x$ on $\mathcal{U}_{M}$.

Proof. As a $C^{3}$ submanifold of $\mathbb{R}^{d}, \Gamma$ can be locally represented as the graph of a $C^{3}$ function. More precisely, for each $x \in \Gamma$, there exists a neighborhood $\mathcal{U}_{x} \subset \mathbb{R}^{d}$ of $x$, an index $j(x) \in\{1, \ldots, d\}$ and a $C_{b}^{3}\left(\mathbb{R}^{d-1}\right)$ function $\varphi_{x}$ such that for all $y \in \Gamma \cap \overline{\mathcal{U}_{x}}, y_{j(x)}=\varphi_{x}\left(y^{(j(x))}\right)$, where $x^{(j)}=\left(x_{1}, \ldots, x_{j-1}, x_{j+1}, \ldots, x_{d}\right)$ for all $1 \leq j \leq d$. Without loss of generality, we can assume that $\mathcal{U}_{x} \subset \mathcal{N}$ and that $\pi\left(\mathcal{U}_{x}\right) \subset \mathcal{U}_{x}$ for all $x \in \Gamma$ (it suffices to replace the set $\mathcal{U}_{x}$ by $\left.\mathcal{U}_{x}^{\prime}=\left\{x \in \mathcal{N}: \pi(x) \in \mathcal{U}_{x} \cap \Gamma\right\}\right)$.

Since $\Gamma$ is compact, there exists $x_{1}, \ldots, x_{M-1}$ in $\Gamma$ such that $\Gamma \subset \cup_{i=1}^{M-1} \mathcal{U}_{i}$, where $\mathcal{U}_{i}=\mathcal{U}_{x_{i}}$. For all $1 \leq i \leq$ $M-1$, let $j_{i}:=j\left(x_{i}\right), \varphi_{i}:=\varphi_{x_{i}}$ and define

$$
\psi_{i}(x):=\left(\rho(x), \pi^{\left(j_{i}\right)}(x)\right)=\left(\rho(x), \pi_{1}(x), \ldots, \pi_{j_{i}-1}(x), \pi_{j_{i}+1}(x), \ldots, \pi_{d}(x)\right), \quad \forall x \in \mathcal{U}_{i}
$$

Now,

$$
x=\pi(x)+\rho(x) n \circ \pi(x), \quad \forall x \in \mathcal{N} .
$$

Therefore, since $\pi \in C_{b}^{2}(\mathcal{N})$ and

$$
\varphi_{i}\left(\pi_{1}(x), \ldots, \pi_{j_{i}-1}(x), \pi_{j_{i}+1}(x), \ldots, \pi_{d}(x)\right)=\pi_{j_{i}}(x), \quad \forall x \in \mathcal{U}_{i},
$$

one can check that $\psi_{i}$ is a $C^{2}$ diffeomorphism from $\mathcal{U}_{i}$ to $\psi_{i}\left(\mathcal{U}_{i}\right)$ with inverse map

$$
\left(\psi_{i}\right)^{-1}\left(y_{1}, \ldots, y_{d}\right)=\tilde{\varphi}_{i}\left(y_{2}, \ldots, y_{d}\right)+y_{1} n\left(\tilde{\varphi}_{i}\left(y_{2}, \ldots, y_{d}\right)\right)
$$

where $\tilde{\varphi}_{i}\left(y^{\prime}\right)=\left(y_{1}^{\prime}, \ldots, y_{j_{i}-1}^{\prime}, \varphi_{i}\left(y^{\prime}\right), y_{j_{i}}^{\prime}, \ldots, y_{d-1}^{\prime}\right)$ for all $y^{\prime} \in \mathbb{R}^{d-1}$. This immediately implies (2.4). Moreover, (2.8) defines $\psi_{i}$ as a $C_{b}^{2}$ function on $\overline{\mathcal{U}}_{i}$.

Therefore, it only remains to check (2.3). This is clearly a consequence of the formula

$$
J \pi(x) n \circ \pi(x)=0, \quad \forall x \in \mathcal{N},
$$

which can be proved as follows. Observe first that, by (2.1), $J(n \circ \pi)(x) n \circ \pi(x)=\nabla^{2} \rho(x) \nabla \rho(x)$, where $\nabla^{2} \rho(x)$ denotes the Hessian matrix of $\rho$ at $x$. The $i$-th coordinate of this vector is

$$
\sum_{j=1}^{d} \frac{\partial^{2} \rho}{\partial x_{i} \partial x_{j}}(x) \frac{\partial \rho}{\partial x_{j}}(x)=\frac{1}{2} \frac{\partial}{\partial x_{i}}\left(\|\nabla \rho(x)\|^{2}\right)=0
$$

which entails $J(n \circ \pi)(x) n \circ \pi(x)=0$. It then follows from (2.9) and (2.1) that

$$
J \pi(x) n \circ \pi(x)=n \circ \pi(x)-J(\rho n \circ \pi)(x) n \circ \pi(x)=-\rho(x) J(n \circ \pi)(x) n \circ \pi(x)=0,
$$

which ends the proof of Proposition 2.1. 


\subsection{A martingale problem}

When the coefficients of an elliptic operator are locally bounded functions, the probabilistic interpretation of elliptic and parabolic PDEs driven by this operator relies on the Stroock and Varadhan martingale problem (see [36]): one needs to prove the existence and uniqueness of the family of probability measures solving this problem, and that this solution enjoys the strong Markov property. Here, due to the discontinuity of $\varepsilon(x)$, expanding the derivative in the definition of the operator $\mathcal{L}$ would formally lead to some of the coefficients equal to measures. This leads us to modify the statement of the classical martingale problem. The proof of existence and uniqueness of the solution needs also specific arguments: see our comments below.

In our context a relevant martingale problem is as follows. Let $\left(\mathcal{C}, \mathcal{B},\left(\mathcal{B}_{t}, t \geq 0\right)\right)$ be the set $\mathcal{C}$ of continuous functions $w$ from $[0,+\infty)$ to $\mathbb{R}^{d}$ endowed with the Borel cylindrical $\sigma$-field $\mathcal{B}$ and the canonical filtration $\left(\mathcal{B}_{t}, t \geq 0\right)$.

\section{Definition 2.2.}

(a) A family of probability measures $\left(\mathbb{P}_{x}\right)_{x \in \mathbb{R}^{d}}$ on $(\mathcal{C}, \mathcal{B})$ solves the martingale problem for the operator $\mathcal{L}$ if, for all $x \in \mathbb{R}^{d}$, one has

and, for all $\varphi$ satisfying

$$
\mathbb{P}_{x}\{w \in \mathcal{C}: w(0)=x\}=1
$$

$$
\begin{gathered}
\varphi \in C_{b}^{0}\left(\mathbb{R}^{d}\right) \cap C_{b}^{2}\left(\mathbb{R}^{d} \backslash \Gamma\right), \\
\varepsilon \nabla \varphi \cdot(n \circ \pi) \in C_{b}^{0}(\mathcal{N}),
\end{gathered}
$$

one has

the process $M_{t}^{\varphi}(w):=\varphi(w(t))-\varphi(w(0))-\int_{0}^{t} \mathcal{L} \varphi(w(s)) \mathrm{d} s \quad$ is a $\mathbb{P}_{x}-\left(\mathcal{B}_{t}\right)$ martingale.

(b) The martingale problem is well-posed if there exists a unique family of probability measure $\left(\mathbb{P}_{x}\right)_{x \in \mathbb{R}^{d}}$ which solves the martingale problem for the operator $\mathcal{L}$.

Remark 2.3. The equality (2.13) means that

$$
\varepsilon_{\text {int }} \nabla^{\text {int }} \varphi(x) \cdot n(x)=\varepsilon_{\text {ext }} \nabla^{\text {ext }} \varphi(x) \cdot n(x), \quad \forall x \in \Gamma,
$$

where

$$
\nabla^{\mathrm{int}} \varphi(x):=\lim _{y \in \Omega_{\mathrm{int}}, y \rightarrow x} \nabla \varphi(y) \quad \text { and } \quad \nabla^{\mathrm{ext}} \varphi(x):=\lim _{y \in \Omega_{\mathrm{ext}}, y \rightarrow x} \nabla \varphi(y), \quad \forall x \in \Gamma .
$$

Note that these two functions are well defined under assumption (2.12). Note also that the transmission property (2.15) has strong links with the operator $\mathcal{L}$, as it is satisfied by the solutions of linear elliptic PDEs involving the operator $\mathcal{L}$ under very general conditions [12].

For SDEs associated to elliptic operators $\mathcal{L}$ with locally bounded coefficients, the set of test functions $\varphi$ can be chosen as the class of $C_{b}^{\infty}$ functions, or the class of continuous bounded functions $\varphi$ such that $\mathcal{L} \varphi$ is continuous and bounded (see, e.g., $[7,10,11,28,36]$ ). In our case, one of the difficulties in solving the martingale problem is that the function $\mathcal{L} \varphi$ cannot be continuous except for very specific $\varphi$. However, if $\varphi$ satisfies the condition (2.12), it is clear that $\mathcal{L} \varphi$ is well-defined and continuous on $\mathbb{R}^{d} \backslash \Gamma$. Now, from Green's identity, for all $\psi \in C_{0}^{1}\left(\mathbb{R}^{d}\right)$, we have

$$
\begin{aligned}
\int_{\mathbb{R}^{d}} \psi \mathcal{L} \varphi & =-\int_{\Omega_{\mathrm{ext}}} \varepsilon \nabla \psi \cdot \nabla \varphi-\int_{\Omega_{\mathrm{int}}} \varepsilon \nabla \psi \cdot \nabla \varphi \\
& =\varepsilon_{\mathrm{ext}} \int_{\Omega_{\mathrm{ext}}} \psi \Delta \varphi+\varepsilon_{\mathrm{int}} \int_{\Omega_{\mathrm{int}}} \psi \Delta \varphi+\oint_{\Gamma} \varepsilon_{\mathrm{int}} \psi \nabla^{\mathrm{int}} \varphi \cdot n-\oint_{\Gamma} \varepsilon_{\mathrm{ext}} \psi \nabla^{\mathrm{ext}} \varphi \cdot n .
\end{aligned}
$$


Thus, if $\varphi$ satisfies condition (2.15), we have

$$
\|\psi \mathcal{L} \varphi\|_{L^{1}\left(\mathbb{R}^{d}\right)} \leq C\|\psi\|_{L^{1}\left(\mathbb{R}^{d}\right)},
$$

for a constant $C$ which only depends on $\varphi$. This means that $\mathcal{L} \varphi$, defined in the sense of the distributions, is $L^{\infty}\left(\mathbb{R}^{d}\right)$ with norm less than $C$. This observation is crucial in the sequel.

We now proceed to the proof of the existence of a solution to the martingale problem. To this end we construct a weak solution to a stochastic differential equation with weighted local time at the boundary $\Gamma$.

\subsection{Existence of a weak solution to a SDE with weighted local time at $\Gamma$}

For all $x$ in $\mathbb{R}^{d}$ consider the SDE

$$
\left\{\begin{array}{l}
X_{t}=x+\int_{0}^{t} \sqrt{2 \varepsilon\left(X_{\theta}\right)} \mathrm{d} B_{\theta}+\frac{\varepsilon_{\text {ext }}-\varepsilon_{\text {int }}}{2 \varepsilon_{\text {ext }}} \int_{0}^{t} n\left(X_{\theta}\right) \mathrm{d} L_{\theta}^{0}(Y), \\
Y_{t}=\rho\left(X_{t}\right) .
\end{array}\right.
$$

Here, $L^{0}(Y)$ stands for the local time at point 0 of the continuous semimartingale $Y$. Notice that the integral $\int_{0}^{t} n\left(X_{\theta}\right) \mathrm{d} L_{\theta}^{0}(Y)$ is well defined since the Stieljes measure $\mathrm{d} L_{\theta}^{0}(Y)$ increases only when $X_{\theta}$ belongs to $\Gamma$.

In this subsection we exhibit a weak solution to (2.17), which means that there exist a filtered probability space equipped with a probability measure $\mathbb{P}_{x}$, a standard $d$-dimensional Brownian motion $\left(B_{t}, t \geq 0\right)$ and a continuous process $\left(X_{t}, t \geq 0\right)$ on this space such that (2.17) holds true for all $t>0, \mathbb{P}_{x}$-a.s. We emphasize that (2.17) is not a classical stochastic differential equation for two reasons: first, the diffusion coefficient is discontinuous; second, the dynamics of $\left(X_{t}\right)$ is driven by the weighted local time of the auxiliary process $\left(Y_{t}\right)$. In particular, the process $\left(X_{t}\right)$ is not at all a straightforward extension of the classical one dimensional skew Brownian motion.

Theorem 2.4. Assume that $\Gamma$ is a compact $C^{3}$ submanifold of $\mathbb{R}^{d}$. Then there exists a weak solution to the $S D E(2.17)$.

Proof. To simplify the notation we limit ourselves to the dimension $d=2$. The generalization to an arbitrary dimension $d \geq 1$ is straightforward.

Let $B$ be a 2-dimensional Brownian motion on a given filtered probability space $\left(\Omega, \mathcal{F},\left(\mathcal{F}_{t}\right)_{t>0}, \mathbb{P}\right)$, and let $W$ be an independent one-dimensional Brownian motion on the same space. Recall definitions (2.6) and (2.7) of $\varepsilon^{\#}$ and $\bar{\psi}_{i}$.

- Fix $1 \leq i \leq M-1, z \in \mathcal{V}_{i}$ and consider the following SDE with initial condition $z$ :

$$
\begin{aligned}
\mathrm{d} \xi_{t}^{1} & =\sqrt{\frac{2}{\varepsilon^{\#}\left(\xi_{t}^{1}\right)}} \mathrm{d} W_{t}, \\
\mathrm{~d} \xi_{t}^{2} & =\sqrt{2 \varepsilon^{\#}\left(\xi_{t}^{1}\right)} \nabla \bar{\psi}_{i}^{2}\left(\left(\bar{\psi}_{i}\right)^{-1}\left(\xi_{t}\right)\right) \cdot \mathrm{d} B_{t},
\end{aligned}
$$

where $\xi_{t}:=\left(\xi_{t}^{1}, \xi_{t}^{2}\right)$. Note that the function $\nabla \bar{\psi}_{i}^{2} \circ\left(\bar{\psi}_{i}\right)^{-1}$ is only defined on $\overline{\mathcal{V}_{i}}$, but, since it is actually $C_{b}^{1}$ on this closed set, it may be extended on $\mathbb{R}^{2}$ as a $C_{b}^{1}\left(\mathbb{R}^{2}\right)$ function. We still denote by $\nabla \bar{\psi}_{i}^{2} \circ\left(\bar{\psi}_{i}\right)^{-1}$ such an extension. Then, the SDE (2.18)-(2.19) is well-defined on $\mathbb{R}^{2}$. Note that the specific choice of this extension is arbitrary and plays no role in our proof.

Although the diffusion coefficient is discontinuous, strong existence and pathwise uniqueness are known for the one-dimensional SDE (2.18) (cf. e.g. [11]). Moreover, the diffusion coefficients of (2.19) have a discontinuous dependency in $\xi_{t}^{1}$ but a Lipschitz dependency in $\xi_{t}^{2}$. Therefore the SDE (2.19) also has a unique strong solution (see, e.g., [36], Thm. 5.1.1). 
- In the case where $i=M$, for all $z \in \mathcal{V}_{M}=\mathcal{U}_{M}$, we consider the following SDE with initial condition $z$ :

$$
\mathrm{d} \xi_{t}=\sqrt{2 \hat{\varepsilon}\left(\xi_{t}\right)} \mathrm{d} B_{t},
$$

where $\hat{\varepsilon} \in C_{b}^{\infty}\left(\mathbb{R}^{2}\right)$ and $\hat{\varepsilon}(x)=\varepsilon(x)$ for all $x \in \mathcal{U}_{M}$. Strong existence and pathwise uniqueness obviously hold true for this SDE.

We can now start the construction of a weak solution to (2.17). Given the initial condition $x$, let

$$
i_{1}:=\min \left\{i \in\{1, \ldots, M\}: \mathrm{d}\left(x, \mathcal{U}_{i}^{c}\right)=\max _{1 \leq j \leq M} \mathrm{~d}\left(x, \mathcal{U}_{j}^{c}\right)\right\},
$$

be the smallest integer $i$ such that the distance between $x$ and $\mathcal{U}_{i}^{c}$ is maximal. Let $Z^{(1)}$ be the solution of (2.18)-(2.19) with $i=i_{1}$ if $i_{1} \leq M-1$ or of (2.20) if $i_{1}=M$, such that $Z_{0}^{(1)}=\bar{\psi}_{i_{1}}(x)$. Consider the stopping time $^{4}$

Then set

$$
\tau_{1}:=\inf \left\{t \geq 0: Z_{t}^{(1)} \notin \mathcal{V}_{i_{1}}\right\} \wedge 1
$$

$$
X_{t}:=\left(\bar{\psi}_{i_{1}}\right)^{-1}\left(Z_{t}^{(1)}\right), \quad \forall t \leq \tau_{1} .
$$

The process $X$ is then constructed inductively as follows: assume that it has been constructed until the time $\tau_{k}$ with $k \geq 1$, and define the random variable

$$
i_{k+1}:=\min \left\{i \in\{1, \ldots, M\}: \mathrm{d}\left(X_{\tau_{k}}, \mathcal{U}_{i}^{c}\right)=\max _{1 \leq j \leq M} \mathrm{~d}\left(X_{\tau_{k}}, \mathcal{U}_{j}^{c}\right)\right\} .
$$

Let $Z^{(k+1)}$ be the solution of (2.18)-(2.19) with $i=i_{k+1}$ if $i_{k+1} \leq M-1$ or of (2.20) if $i_{k+1}=M$, with initial condition $Z_{\tau_{k}}^{(k+1)}=\bar{\psi}_{i_{k+1}}\left(X_{\tau_{k}}\right)$ at time $\tau_{k}$. Define

$$
\tau_{k+1}:=\inf \left\{t \geq \tau_{k}: Z_{t}^{\left(i_{k+1}\right)} \notin \mathcal{V}_{i_{k+1}}\right\} \wedge\left(\tau_{k}+1\right),
$$

and

$$
X_{t}:=\left(\bar{\psi}_{i_{k+1}}\right)^{-1}\left(Z_{t}^{(k+1)}\right), \quad \forall \tau_{k} \leq t \leq \tau_{k+1} .
$$

Suppose that we have proven that the increasing sequence $\tau_{k}$ almost surely converges to $+\infty$ (see Lem. 2.5 below). Then the process $X$ is a.s. well defined on all finite time interval.

Now, since $X_{t}=\left(\bar{\psi}_{i_{k}}\right)^{-1}\left(Z_{t}^{(k)}\right)=F^{-1} \circ\left(\psi_{i_{k}}\right)^{-1}\left(Z_{t}^{(k)}\right)$ for all $\tau_{k-1} \leq t \leq \tau_{k}$, applying first Itô's formula to $\left(\psi_{i_{k}}\right)^{-1}$ and next Itô-Tanaka's formula to $F^{-1}$ we have

$$
\begin{aligned}
\mathrm{d} X_{t}= & \sqrt{2 \varepsilon\left(X_{t}\right)} \mathrm{d} \hat{B}_{t}+\frac{\varepsilon_{\text {ext }}-\varepsilon_{\text {int }}}{2 \varepsilon_{\text {ext }}} n\left(X_{t}\right) \mathrm{d} L_{t}^{0}\left(Y_{t}\right)+\varepsilon\left(X_{t}\right) \sum_{k=1}^{\infty} \mathbb{1}_{\left\{\tau_{k-1} \leq t<\tau_{k}\right\}} \mathbb{1}_{\left\{i_{k} \neq M\right\}}\left(\frac{\partial^{2}\left(\psi_{i_{k}}\right)^{-1}}{\partial x_{1}^{2}}\left(\psi_{i_{k}}\left(X_{t}\right)\right)\right. \\
& \left.+\frac{\partial^{2}\left(\psi_{i_{k}}\right)^{-1}}{\partial x_{2}^{2}}\left(\psi_{i_{k}}\left(X_{t}\right)\right)\left|\nabla \psi_{i_{k}}^{2}\left(X_{t}\right)\right|^{2}\right) \mathrm{d} t
\end{aligned}
$$

for all $t \geq 0$, where $Y_{t}:=\rho\left(X_{t}\right)$, with the convention that $\tau_{0}=0$.

The second term of the right-hand side is a consequence of (2.4). The two-dimensional process $\hat{B}$ in the first term of the right-hand side of (2.22) is defined as follows: $\hat{B}_{0}=0$ and, for any $k \geq 1$ and for $\tau_{k-1} \leq t \leq \tau_{k}$,

$$
\hat{B}_{t}=\hat{B}_{\tau_{k-1}}+\mathbb{1}_{\left\{i_{k} \leq M-1\right\}} \int_{\tau_{k-1}}^{t} J\left(\left(\psi_{i_{k}}\right)^{-1}\right)\left(Z_{s}^{(k)}\right)\left(\begin{array}{l}
\mathrm{d} W_{s} \\
\nabla \psi_{i_{k}}^{2}\left(\left(\psi_{i_{k}}\right)^{-1}\left(Z_{s}^{(k)}\right)\right) \cdot \mathrm{d} B_{s}
\end{array}\right)+\mathbb{1}_{\left\{i_{k}=M\right\}}\left(B_{t}-B_{\tau_{k-1}}\right) .
$$

\footnotetext{
${ }^{4}$ By definition $\tau_{1} \leq 1$ a.s.: this bound avoids us, here and in the sequel, to distinguish the cases $\tau_{1}$ is finite or infinite, which simplifies details in the proofs of Lemma 2.5 and Theorem 2.12.
} 
It follows easily from the equality $J\left(\psi_{m}^{-1}\right)\left(\psi_{m}(x)\right) J\left(\psi_{m}\right)(x)=\operatorname{Id}$ for all $x \in \mathcal{U}_{m}$ and $1 \leq m \leq M-1$ that, for all $k \geq 1$ and $\tau_{k-1} \leq t<\tau_{k}$,

$$
\mathrm{d} \hat{B}_{t}=\mathrm{d} B_{t}+\mathbb{1}_{\left\{i_{k} \leq M-1\right\}}\left(-n\left(\pi\left(X_{t}\right)\right)\left(n\left(\pi\left(X_{t}\right)\right) \cdot \mathrm{d} B_{t}\right)+n\left(\pi\left(X_{t}\right)\right) \mathrm{d} W_{t}\right),
$$

which implies that $\hat{B}$ is a standard Brownian motion in $\mathbb{R}^{2}$ by Lévy's theorem ([11], Thm. 3.3.16).

Finally, since $\varepsilon(\cdot)$ is bounded away from zero and the last drift term of the $\operatorname{SDE}(2.22)$ is bounded, it classically follows from Girsanov's theorem that, for all $0 \leq t \leq T$, there exists a $\mathbb{Q}$-Brownian motion $\tilde{B}$, where $\mathbb{Q}$ is a probability measure on $\mathcal{F}_{T}$ equivalent to $\mathbb{P}$, such that $X$ solves up to time $T$ equation (2.17) driven by the Brownian motion $\tilde{B}$. This ends the construction of a weak solution to (2.17).

In the preceding proof we have admitted the following lemma.

Lemma 2.5. The increasing sequence $\tau_{k}$ almost surely converges to $+\infty$.

Proof. Here again, we will assume that $d=2$ for simplicity. The argument obviously extends to higher dimensions.

For any $i \in\{1, \ldots, M\}$ and any $x \in \partial \mathcal{U}_{i}, \sup _{j \neq i} \mathrm{~d}\left(x, \mathcal{U}_{j}^{c}\right)$ is positive and continuous with respect to $x \in \partial \mathcal{U}_{i}$. Since $\mathcal{U}_{i}$ is bounded for $1 \leq i \leq M-1$ and $\partial \mathcal{U}_{M} \subset \bigcup_{i=1}^{n-1} \mathcal{U}_{i}$, the set $\partial \mathcal{U}_{i}$ is compact for any $i \in\{1, \ldots, M\}$. Hence,

$$
\rho:=\inf _{1 \leq i \leq M} \inf _{x \in \partial \mathcal{U}_{i}} \sup _{j \neq i} \mathrm{~d}\left(x, \mathcal{U}_{j}^{c}\right)>0 .
$$

Then, it is clear from the definition of $i_{k}$ and $\tau_{k}$ that either $\tau_{k}=\tau_{k-1}+1$ or $\left\|X_{\tau_{k}}-X_{\tau_{k-1}}\right\| \geq \rho$. The idea of this proof is to prove that, almost surely, $\tau_{k}-\tau_{k-1} \geq T$ infinitely often w.r.t. $k \geq 1$ for $T$ small enough. Without the local time and drift terms and if the diffusion coefficient were constant in (2.22), this event would be implied by the event $\left\{\sup _{t \in\left[\tau_{k-1}, \tau_{k-1}+T\right]}\left|B_{t}-B_{\tau_{k-1}}\right| \leq \rho^{\prime}\right.$ infinitely often $\}$ for some $\rho^{\prime}>0$, which has probability one by Borel-Cantelli's lemma and the strong Markov property of the Brownian motion. In our case, we use a similar argument, but with a more involved justification.

In order to get rid of the local time term, we use the sequence of processes $\left(Z^{(k)}\right)_{k}$ defined in the proof of Theorem 2.4. If $T<1$, the event $\left\{\tau_{k}-\tau_{k-1} \geq T\right\}$ is implied by the event

$$
\left\{\sup _{0 \leq t \leq T}\left|Z_{\tau_{k-1}+t}^{(k)}-Z_{\tau_{k-1}}^{(k)}\right| \leq \rho^{\prime}\right\}
$$

for some $\rho^{\prime}>0$ depending on $\rho$ and on $\max _{1 \leq i \leq M} \max _{x \in \mathcal{V}_{i}} J\left(\bar{\psi}_{i}\right)^{-1}(x)$.

Now, $Z^{(k)}$ solves (2.18)-(2.19) for $i=i_{k}$ if $i_{k} \leq M-1$, or (2.20) if $i_{k}=M$. In both cases, the event (2.23) is implied by the event

$$
A_{k}:=\bigcap_{j=1}^{3}\left\{\sup _{0 \leq t \leq T}\left|\int_{\tau_{k-1}}^{\tau_{k-1}+t} H_{s}^{(k), j} \mathrm{~d} \tilde{W}_{s}^{j}\right| \leq \rho^{\prime \prime}\right\}
$$

for $\rho^{\prime \prime}>0$ depending only on $\rho^{\prime}$, where $\tilde{W}=\left(\tilde{W}^{1}, \tilde{W}^{2}, \tilde{W}^{3}\right)$ is the 3-dimensional Brownian motion $\left(W_{t}, B_{t}^{1}\right.$, $\left.B_{t}^{2}\right)_{t \geq 0}$ and

$$
\begin{aligned}
\forall t \geq 0, \quad H_{t}^{(k)}=\left(H_{t}^{(k), 1}, H_{t}^{(k), 2}, H_{t}^{(k), 3}\right)= & \mathbb{1}_{\left\{\tau_{k-1} \leq t<\tau_{k}\right\}} \sqrt{2 \varepsilon\left(X_{t}\right)} \\
& \times\left[\left(1 / \varepsilon\left(X_{t}\right), \frac{\partial \bar{\psi}_{i_{k}}^{2}}{\partial x_{1}}\left(X_{t}\right), \frac{\partial \bar{\psi}_{i_{k}}^{2}}{\partial x_{2}}\left(X_{t}\right)\right) \mathbb{1}_{\left\{i_{k} \leq M-1\right\}}+(0,1,1) \mathbb{1}_{\left\{i_{k}=M\right\}}\right] .
\end{aligned}
$$


The processes $\left(H_{t}^{(k)}\right)_{t \geq 0}$ are $\mathcal{F}_{t}$-adapted and a.s. uniformly bounded by, say, $\bar{H}$. Therefore, for any $B \in \mathcal{F}_{\tau_{k-1}}$ and $j=1,2,3$, the process

$$
\left(\int_{\tau_{k-1}}^{\tau_{k-1}+t} \mathbb{1}_{B} H_{s}^{(k), j} \mathrm{~d} \tilde{W}_{s}^{j}, t \geq 0\right)
$$

is a $\left(\mathcal{F}_{\tau_{k-1}+t}\right)_{t \geq 0}$ martingale, and by Doob's inequality,

$$
\rho^{\prime \prime 2} \mathbb{P}\left(A_{k}^{c} \cap B\right) \leq \sum_{j=1}^{3} \mathbb{E}\left[\sup _{0 \leq t \leq T}\left(\int_{\tau_{k-1}}^{\tau_{k-1}+t} \mathbb{1}_{B} H_{s}^{(k), j} \mathrm{~d} \tilde{W}_{s}^{j}\right)^{2}\right] \leq 12 \bar{H} T \mathbb{P}(B) .
$$

Choosing $B=\left\{\mathbb{P}\left(A_{k}^{c} \mid \mathcal{F}_{\tau_{k-1}}\right)>12 \bar{H} T / \rho^{\prime \prime 2}\right\}$ in the previous inequality, we obtain that, for all $k \geq 1$, $\mathbb{P}\left(A_{k}^{c} \mid \mathcal{F}_{\tau_{k-1}}\right) \leq 12 \bar{H} T / \rho^{\prime \prime 2}$ a.s. This constant can be made smaller than 1 by taking $T$ small enough.

Since moreover $A_{k} \in \mathcal{F}_{\tau_{k}}$ for all $k \geq 1$, this implies that, for all $m<n$,

$$
\mathbb{P}\left(\bigcap_{m \leq k \leq n} A_{k}^{c}\right)=\mathbb{E}\left(\mathbb{1}_{\bigcap_{m \leq k \leq n-1} A_{k}^{c}} \mathbb{P}\left(A_{n}^{c} \mid \mathcal{F}_{\tau_{n-1}}\right)\right) \leq \ldots \leq\left(\frac{12 \bar{H} T}{\rho^{\prime \prime 2}}\right)^{n-m+1},
$$

and thus

$$
\lim _{n \rightarrow+\infty} \mathbb{P}\left(\bigcap_{m \leq k \leq n} A_{k}^{c}\right)=0, \quad \forall m \geq 1 .
$$

This entails that the events $A_{k}$ a.s. occur infinitely often. Therefore, we found $T>0$ such that the events $\left\{\tau_{k}-\tau_{k-1} \geq T\right\}$ a.s. occur infinitely often, which ends the proof of Lemma 2.5 .

The previous proof can be extended to prove the next result.

Lemma 2.6. For any weak solution $X$ of (2.17), we define inductively the sequence $\left(\tau_{k}\right)_{k \geq 1}$ by

$$
\tau_{k+1}:=\inf \left\{t \geq i_{k}: X_{t} \notin \mathcal{U}_{i_{k+1}}\right\} \wedge\left(\tau_{k}+1\right),
$$

where the random integers $\left(i_{k}\right)_{k \geq 1}$ are defined as in the proof of Theorem 2.4. Then, the sequence $\left(\tau_{k}\right)_{k \geq 1}$ converges to $+\infty$ almost surely.

In order to prove that the probability law of the process $X$ solves the martingale problem of Definition 2.2 we need to establish a version of the classical Itô's formula which is adapted to our needs.

\subsection{A generalized Itô-Meyer formula}

As mentioned in Remark 2.3, the solution $u$ of the Poisson-Boltzmann equation (1.2) (and more generally, the solutions of linear elliptic PDEs involving the operator $\mathcal{L}$ ) has discontinuous normal gradient on $\Gamma$. Therefore, one cannot apply Itô's formula to $u\left(X_{t}\right)$. In the one-dimensional case, this could be done thanks to Itô-Meyer's formula ([26], Thm. IV.70). The main result of this section extends this formula to higher dimensions and to functions satisfying the transmission property (2.15).

We start with the following lemma.

Lemma 2.7. Let $X$ be a weak solution of (2.17). Let $\tilde{u}$ be a function in $W_{\text {loc }}^{2, \infty}\left(\mathbb{R}^{d}\right) \cap C^{2}\left(\mathbb{R}^{d} \backslash \Gamma\right)$. Then, a.s. for all $t \geq 0$,

$$
\tilde{u}\left(X_{t}\right)=\tilde{u}\left(X_{0}\right)+\int_{0}^{t} \nabla \tilde{u}\left(X_{s}\right) \cdot \mathrm{d} X_{s}+\int_{0}^{t} \mathcal{L} \tilde{u}\left(X_{s}\right) \mathbb{1}_{\left\{X_{s} \notin \Gamma\right\}} \mathrm{d} s .
$$


Proof. Note first that, by classical localization techniques, it is enough to prove this result for $\tilde{u}$ with compact support. Note also that $\tilde{u} \in W^{2, \infty}\left(\mathbb{R}^{d}\right)$ implies that $\tilde{u} \in C^{1}\left(\mathbb{R}^{d}\right)$. We consider a sequence $\left(\tilde{u}_{n}\right)_{n \geq 1}$ of functions in $C^{2}\left(\mathbb{R}^{d}\right)$ (obtained for example by convolution) such that

$$
\left\{\begin{array}{l}
\lim _{n \rightarrow 0}\left\|\tilde{u}_{n}-\tilde{u}\right\|_{L^{\infty}\left(\mathbb{R}^{d}\right)}=0, \\
\lim _{n \rightarrow 0}\left\|\nabla \tilde{u}_{n}-\nabla \tilde{u}\right\|_{L^{\infty}\left(\mathbb{R}^{d}\right)}=0, \\
\lim _{n \rightarrow \infty} \nabla^{2} \tilde{u}_{n}(x)=\nabla^{2} \tilde{u}(x), \quad \forall x \in \mathbb{R}^{d} \backslash \Gamma, \\
\sup _{n \geq 1}\left\|\nabla^{2} \tilde{u}_{n}\right\|_{L^{\infty}\left(\mathbb{R}^{d}\right)}<\infty,
\end{array}\right.
$$

where $\nabla^{2} \tilde{u}$ stands for the Hessian matrix of $\tilde{u}$.

Applying Itô's formula to $\tilde{u}_{n}\left(X_{t}\right)$ yields

$$
\tilde{u}_{n}\left(X_{t}\right)=\tilde{u}_{n}\left(X_{0}\right)+\int_{0}^{t} \nabla \tilde{u}_{n}\left(X_{s}\right) \cdot \mathrm{d} X_{s}+\int_{0}^{t} \varepsilon\left(X_{s}\right) \Delta \tilde{u}_{n}\left(X_{s}\right) \mathrm{d} s .
$$

We need to prove the a.s. convergence, when $n \rightarrow+\infty$, of each terms in the previous equation.

Since $L_{t}^{0}(Y)$ has finite variation, it follows from $(2.25)$ that

$$
\lim _{n \rightarrow \infty} \int_{0}^{t} \nabla \tilde{u}_{n}\left(X_{s}\right) \cdot n\left(\pi\left(X_{s}\right)\right) \mathrm{d} L_{s}^{0}(Y)=\int_{0}^{t} \nabla \tilde{u}\left(X_{s}\right) \cdot n\left(\pi\left(X_{s}\right)\right) \mathrm{d} L_{s}^{0}(Y) \quad \text { a.s. }
$$

and

$$
\mathbb{E}\left|\int_{0}^{t} \sqrt{2 \varepsilon\left(X_{s}\right)}\left(\nabla \tilde{u}_{n}\left(X_{s}\right)-\nabla \tilde{u}\left(X_{s}\right)\right) \cdot \mathrm{d} B_{s}\right|^{2} \leq C\left\|\nabla \tilde{u}_{n}-\nabla \tilde{u}\right\|_{L^{\infty}\left(\mathbb{R}^{d}\right)}^{2} t
$$

which implies the a.s. convergence of a sub-sequence of $\int_{0}^{t} \sqrt{2 \varepsilon\left(X_{s}\right)} \nabla \tilde{u}_{n}\left(X_{s}\right) \cdot \mathrm{d} B_{s}$ to $\int_{0}^{t} \sqrt{2 \varepsilon\left(X_{s}\right)} \nabla \tilde{u}\left(X_{s}\right) \cdot \mathrm{d} B_{s}$.

For the second-order term, (2.25) and Lebesgue's dominated convergence theorem imply that

$$
\lim _{n \rightarrow \infty} \int_{0}^{t} \mathbb{1}_{\left\{X_{s} \notin \Gamma\right\}} \varepsilon\left(X_{s}\right) \Delta \tilde{u}_{n}\left(X_{s}\right) \mathrm{d} s=\int_{0}^{t} \mathbb{1}_{\left\{X_{s} \notin \Gamma\right\}} \varepsilon\left(X_{s}\right) \Delta \tilde{u}\left(X_{s}\right) \mathrm{d} s=\int_{0}^{t} \mathbb{1}_{\left\{X_{s} \notin \Gamma\right\}} \mathcal{L} \tilde{u}\left(X_{s}\right) \mathrm{d} s \quad \text { a.s. }
$$

Finally, using the occupation time formula (see, e.g., [27]), we have that for all $n \geq 1$, a.s.

$$
\begin{aligned}
\left|\int_{0}^{t} \mathbb{1}_{\left\{X_{s} \in \Gamma\right\}} \varepsilon\left(X_{s}\right) \Delta \tilde{u}_{n}\left(X_{s}\right) \mathrm{d} s\right| & =\frac{1}{2}\left|\int_{0}^{t} \mathbb{1}_{\left\{Y_{s}=0\right\}} \Delta \tilde{u}_{n}\left(X_{s}\right) \mathrm{d}\langle Y, Y\rangle_{s}\right| \\
& \leq C \int_{0}^{t} \mathbb{1}_{\left\{Y_{s}=0\right\}} \mathrm{d}\langle Y, Y\rangle_{s} \\
& =C \int_{-\infty}^{\infty} \mathbb{1}_{a=0} L_{t}^{a}(Y) \mathrm{d} a \\
& =0 .
\end{aligned}
$$

This ends the proof of Lemma 2.7.

Theorem 2.8. Assume that $\Gamma$ is a compact $C^{3}$ submanifold of $\mathbb{R}^{d}$. Let $u$ be a function on $\mathbb{R}^{d}$ such that there exists a function $f \in W_{\mathrm{loc}}^{2, \infty}\left(\mathbb{R}^{d}\right) \cap C^{2}\left(\mathbb{R}^{d} \backslash \Gamma\right)$ satisfying

$$
\hat{u}(x):=u(x)-f(x)[\rho(x)]_{+} \in W_{\text {loc }}^{2, \infty}\left(\mathbb{R}^{d}\right) \cap C^{2}\left(\mathbb{R}^{d} \backslash \Gamma\right) .
$$


(Recall that $\rho$ has been extended as a $C_{b}^{2}\left(\mathbb{R}^{d}\right)$ function.) As in Remark 2.3 , we set

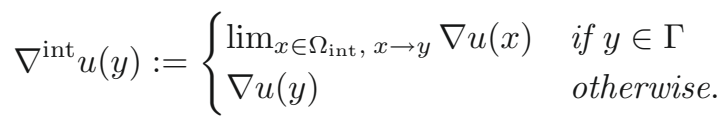

All weak solution to (2.17) satisfies: a.s. for all $t \geq 0$,

$$
\begin{aligned}
u\left(X_{t}\right)= & u\left(X_{0}\right)+\int_{0}^{t} \sqrt{2 \varepsilon\left(X_{s}\right)} \nabla^{\mathrm{int}} u\left(X_{s}\right) \cdot \mathrm{d} B_{s}+\int_{0}^{t} \mathcal{L} u\left(X_{s}\right) \mathbb{1}_{\left\{X_{s} \notin \Gamma\right\}} \mathrm{d} s \\
& +\frac{1}{2} \int_{0}^{t}\left(f\left(X_{s}\right)+\frac{\varepsilon_{\text {ext }}-\varepsilon_{\text {int }}}{\varepsilon_{\text {ext }}} \nabla^{\mathrm{int}} u\left(X_{s}\right) \cdot n\left(X_{s}\right)\right) \mathrm{d} L_{s}^{0}(Y)
\end{aligned}
$$

where $Y_{t}:=\rho\left(X_{t}\right)$.

Proof. Note first that $\nabla^{\text {int }} u$ is well defined since $u(x)=\hat{u}(x)+f(x)[\rho(x)]_{+}$and $\hat{u}$ and $f$ are $C^{1}\left(\mathbb{R}^{d}\right)$ by assumption. Note also that the last term of the right-hand side of (2.28) is well-defined since the local time $L_{s}^{0}(Y)$ only increases when $X_{s}$ belongs to $\Gamma$.

By Itô's formula, $Y$ is a semimartingale such that, almost surely, for all $t \geq 0$,

$$
Y_{t}=Y_{0}+\int_{0}^{t} \nabla \rho\left(X_{s}\right) \cdot \mathrm{d} X_{s}+\int_{0}^{t} \varepsilon\left(X_{s}\right) \Delta \rho\left(X_{s}\right) \mathrm{d} s
$$

Then, it follows from Itô-Tanaka's formula (see, e.g., [27]) that

$$
\left[Y_{t}\right]_{+}=\left[Y_{0}\right]_{+}+\int_{0}^{t} \mathbb{1}_{\left\{Y_{s}>0\right\}} \nabla \rho\left(X_{s}\right) \cdot \mathrm{d} X_{s}+\int_{0}^{t} \mathbb{1}_{\left\{Y_{s}>0\right\}} \varepsilon\left(X_{s}\right) \Delta \rho\left(X_{s}\right) \mathrm{d} s+\frac{1}{2} L_{t}^{0}(Y) .
$$

This yields

$$
\left\langle X^{i},[Y]_{+}\right\rangle_{t}=2 \int_{0}^{t} \varepsilon\left(X_{s}\right) \mathbb{1}_{\left\{Y_{s}>0\right\}} \frac{\partial \rho}{\partial x_{i}}\left(X_{s}\right) \mathrm{d} s, \quad \forall t \geq 0, \quad \text { a.s. }
$$

Applying Lemma 2.7 to $f$, we obtain the decomposition of the semimartingale $f\left(X_{t}\right)$. Applying Itô's formula to the product $f\left(X_{t}\right)\left[Y_{t}\right]_{+}$we obtain

$$
\begin{aligned}
f\left(X_{t}\right)\left[Y_{t}\right]_{+}= & f\left(X_{0}\right)\left[Y_{0}\right]_{+}+\int_{0}^{t}\left[Y_{s}\right]_{+} \nabla f\left(X_{s}\right) \cdot \mathrm{d} X_{s}+\int_{0}^{t} f\left(X_{s}\right) \mathbb{1}_{\left\{Y_{s}>0\right\}} \nabla \rho\left(X_{s}\right) \cdot \mathrm{d} X_{s}+\frac{1}{2} \int_{0}^{t} f\left(X_{s}\right) \mathrm{d} L_{s}^{0}(Y) \\
& +\int_{0}^{t}\left[\varepsilon\left(X_{s}\right) \mathbb{1}_{\left\{Y_{s}>0\right\}} f\left(X_{s}\right) \Delta \rho\left(X_{s}\right)+2 \varepsilon\left(X_{s}\right) \mathbb{1}_{\left\{Y_{s}>0\right\}} \nabla f\left(X_{s}\right) \cdot \nabla \rho\left(X_{s}\right)+\mathbb{1}_{\left\{Y_{s} \neq 0\right\}}\left[Y_{s}\right]_{+} \mathcal{L} f\left(X_{s}\right)\right] \mathrm{d} s .
\end{aligned}
$$

Now,

$$
\nabla^{\mathrm{int}} u(x)=\nabla \hat{u}(x)+\nabla f(x)[\rho(x)]_{+}+f(x) \mathbb{1}_{\{\rho(x)>0\}} \nabla \rho(x)
$$

and

$$
\Delta u(x)=\Delta \hat{u}(x)+\mathbb{1}_{\{\rho(x)>0\}} f(x) \Delta \rho(x)+2 \mathbb{1}_{\{\rho(x)>0\}} \nabla f(x) \cdot \nabla \rho(x)+\Delta f(x)[\rho(x)]_{+} \quad \forall x \in \mathbb{R}^{d} \backslash \Gamma .
$$

To end the proof of Theorem 2.8, it then remains to combine (2.29) and Lemma 2.7 applied to $\hat{u}\left(X_{t}\right)$, and to remind that $\nabla \rho(x)=n(\pi(x))$ when $x \in \mathcal{N}$. 
Corollary 2.9. Let $u$ satisfy (2.12) and (2.13). Then, almost surely, for all $t \geq 0$,

$$
u\left(X_{t}\right)=u\left(X_{0}\right)+\int_{0}^{t} \mathbb{1}_{\left\{X_{s} \notin \Gamma\right\}} \sqrt{2 \varepsilon\left(X_{s}\right)} \nabla u\left(X_{s}\right) \cdot \mathrm{d} B_{s}+\int_{0}^{t} \mathbb{1}_{\left\{X_{s} \notin \Gamma\right\}} \mathcal{L} u\left(X_{s}\right) \mathrm{d} s .
$$

Proof. Assume first, in addition to the assumptions of Corollary 2.9, that

$$
\begin{gathered}
\nabla^{\mathrm{int}} u(x) \cdot n(x) \in C^{2}(\Gamma) \\
\text { and } \quad u \circ\left(\bar{\psi}_{i}\right)^{-1} \in C^{1}\left(\mathcal{V}_{i}\right), \quad \forall 1 \leq i \leq M .
\end{gathered}
$$

Note that, since $u \in C_{b}^{2}\left(\mathbb{R}^{d} \backslash \Gamma\right), \nabla^{\text {int }} u$ is well defined on $\Gamma$. Note also that, by (2.4), for all $i \leq M-1$,

$$
\frac{\partial}{\partial x_{1}}\left(u \circ\left(\bar{\psi}_{i}\right)^{-1}\right)\left(\bar{\psi}_{i}(x)\right)=\varepsilon(x) \nabla u(x) \cdot n \circ \pi(x), \quad \forall x \in \mathcal{U}_{i},
$$

which shows that (2.32) actually implies (2.13).

By assumption (2.31), the function

$$
f(x)=\left(\frac{\varepsilon_{\text {int }}}{\varepsilon_{\text {ext }}}-1\right) \nabla^{\text {int }} u(\pi(x)) \cdot n(\pi(x)), \quad \forall x \in \mathcal{N},
$$

can be extended to a $C^{2}\left(\mathbb{R}^{d}\right)$ function, and the function $\hat{u}$ of $(2.26)$ is continuous and of class $C_{b}^{2}\left(\mathbb{R}^{d} \backslash \Gamma\right)$. For all $1 \leq i \leq M$, define

$$
v_{i}(x):=\hat{u} \circ \psi_{i}^{-1}(x)=u \circ \psi_{i}^{-1}(x)-f \circ \psi_{i}^{-1}(x)\left[\rho \circ \psi_{i}^{-1}(x)\right]_{+}, \quad \forall x \in \psi_{i}\left(\mathcal{U}_{i}\right),
$$

where the function $\psi_{i}$ is defined in Proposition 2.1, and fix $1 \leq i \leq M-1$. We deduce from (2.10) that, as $f(x)$ only depends on $\pi(x), \nabla f(x) \cdot n \circ \pi(x)=0$ for all $x \in \mathcal{N}$. Therefore, it follows from (2.4) and from the fact that $\rho \circ \psi_{i}^{-1}(x)=x_{1}$ that

$$
\frac{\partial v_{i}}{\partial x_{1}}\left(\psi_{i}(x)\right)=\nabla u(x) \cdot n \circ \pi(x)-f(x) \mathbb{1}_{\left\{x \in \Omega_{\text {ext }}\right\}}, \quad \forall x \in \mathcal{U}_{i} \backslash \Gamma .
$$

Since $u$ satisfies (2.13), this function can be extended continuously to $\mathcal{U}_{i}$. Furthermore, for $2 \leq j \leq d$, by definition of the function $F$ in (2.5),

$$
\frac{\partial v_{i}}{\partial x_{j}}\left(\psi_{i}(x)\right)=\frac{\partial}{\partial x_{j}}\left(u \circ\left(\bar{\psi}_{i}\right)^{-1}\right)\left(\bar{\psi}_{i}(x)\right)-\frac{\partial}{\partial x_{j}}\left(f \circ \psi_{i}^{-1}\right)\left(\psi_{i}(x)\right)[\rho(x)]_{+}, \quad \forall x \in \mathcal{U}_{i} \backslash \Gamma .
$$

Again, by (2.32), this defines a continuous function on $\mathcal{U}_{i}$. Thus $v_{i} \in C^{1}\left(\psi_{i}\left(\mathcal{U}_{i}\right)\right)$ for all $1 \leq i \leq M-1$, and the same result is trivial for $i=M$. Since $\psi_{i}$ is a $C_{b}^{2}$ diffeomorphism on $\mathcal{U}_{i}$, we finally deduce that $\hat{u} \in C_{b}^{1}\left(\mathbb{R}^{d}\right)$. As $J \hat{u}$ is differentiable with bounded derivatives almost everywhere in $\mathbb{R}^{d}$, we have $\hat{u} \in W^{2, \infty}\left(\mathbb{R}^{d}\right)$. Thus, Theorem 2.8 applies, and (2.28) yields

$$
u\left(X_{t}\right)=u\left(X_{0}\right)+\int_{0}^{t} \sqrt{2 \varepsilon\left(X_{s}\right)} \nabla^{\mathrm{int}} u\left(X_{s}\right) \cdot \mathrm{d} B_{s}+\int_{0}^{t} \mathbb{1}_{\left\{X_{s} \notin \Gamma\right\}} \mathcal{L} u\left(X_{s}\right) \mathrm{d} s, \quad \text { a.s. } \forall t \geq 0 .
$$

The rest of the proof is devoted to the extension of (2.34) to any function $u$ satisfying only (2.12) and (2.13). First, let us show that it suffices to prove this only for $u$ with compact support in $\mathcal{U}_{i}$ for some $1 \leq i \leq M$. For this, remind from the proof of Proposition 2.1 that the set $\mathcal{U}_{i}$ for $1 \leq i \leq M-1$ can be assumed to be of the form $\left\{x \in \mathcal{N}: \pi(x) \in \mathcal{U}_{i} \cap \Gamma\right\}$. Let $\left(\chi_{i}\right)_{1 \leq i \leq M-1}$ be a $C^{3}$ partition of unity corresponding to the covering of $\Gamma$ 
by $\cup_{i=1}^{M-1} \mathcal{U}_{i} \cap \Gamma$ : for all $1 \leq i \leq M-1, \chi_{i} \in C^{3}(\Gamma)$ with compact support in $\mathcal{U}_{i} \cap \Gamma$ and $\sum_{i=1}^{M-1} \chi_{i}(x)=1$ for all $x \in \Gamma$. Fix also $\chi \in C^{\infty}\left(\mathbb{R}^{d}\right)$ with support having empty intersection with $\Gamma$ and such that $\chi(x)=1$ for all $x \in \mathcal{U}_{M}$. Now, for any $u$ satisfying (2.12) and (2.13), define

$$
u_{i}=(1-\chi) \chi_{i} \circ \pi u, \quad 1 \leq i \leq M-1
$$

and

$$
u_{M}=\chi u
$$

Then, for all $1 \leq i \leq M, u_{i}$ has compact support in $\mathcal{U}_{i}$ and satisfies (2.12) and (2.13), and $\sum_{i=1}^{M} u_{i}=u$. Since the equation (2.34) is linear in $u$, it suffices to prove it for each $u_{i}$.

So let $u \in C_{c}\left(\mathcal{U}_{i}\right)$ satisfy (2.12) and (2.13). The case $i=M$ is trivial, so we assume that $i \leq M-1$. We are going to construct a sequence of functions $\left(u^{(n)}\right)_{n \geq 1}$ satisfying (2.12), (2.13), (2.31) and (2.32) such that one can pass to the limit in (2.34). As in the proof of Theorem 2.4, we assume for simplicity that $d=2$. The proof straightforwardly adapts to higher dimensions.

The function $\tilde{u}=u \circ\left(\bar{\psi}_{i}\right)^{-1}$ is continuous with compact support in $\mathcal{V}_{i}$. Let us extend it to $\mathbb{R}^{2}$ by 0 out of $\mathcal{V}_{i}$. Then, this function is of class $C_{b}^{2}((\mathbb{R} \backslash\{0\}) \times \mathbb{R})$, and it follows from $(2.33)$ that $\partial \tilde{u} / \partial x_{1}$ is continuous on $\mathbb{R}^{2}$. It also follows from elementary computations that $\mathcal{L} u=(\tilde{\mathcal{L}} \tilde{u}) \circ \bar{\psi}_{i}$, where

$$
\tilde{\mathcal{L}} \tilde{u}(x)=\sum_{j=1}^{2} \nabla \cdot\left(\varepsilon \nabla \bar{\psi}_{i}^{j}\right) \circ\left(\bar{\psi}_{i}\right)^{-1}(x) \frac{\partial \tilde{u}}{\partial x_{i}}(x)+\sum_{j, k=1}^{2} \varepsilon^{\#}\left(x_{1}\right) \nabla \bar{\psi}_{i}^{j} \circ\left(\bar{\psi}_{i}\right)^{-1}(x) \cdot \nabla \bar{\psi}_{i}^{k} \circ\left(\bar{\psi}_{i}\right)^{-1}(x) \frac{\partial^{2} \tilde{u}}{\partial x_{i} \partial x_{j}}(x),
$$

where $\varepsilon^{\#}$ has been defined in (2.6). In the previous relation, the functions $\left(\bar{\psi}_{i}\right)^{-1}$ and $J \bar{\psi}_{i}$ may be extended arbitrarily to $\mathbb{R}^{2}$ since $\tilde{u}(x)=0$ for all $x \notin \mathcal{V}_{i}$.

Now, on $\mathcal{U}_{i}, \nabla \cdot\left(\varepsilon \nabla \bar{\psi}_{i}^{1}\right)=\nabla \cdot(n \circ \pi)$ is continuous, and $\nabla \cdot\left(\varepsilon \nabla \bar{\psi}_{i}^{2}\right)=\varepsilon \Delta \bar{\psi}_{i}^{2}+\nabla \varepsilon \cdot \nabla \bar{\psi}_{i}^{2}$, where the last term is a priori only defined in the sense of distributions. However, $\nabla \bar{\psi}_{i}^{2} \cdot(n \circ \pi)=0$ by (2.3), so that $\nabla \varepsilon \cdot \nabla \bar{\psi}_{i}^{2}$ is actually 0 (to be fully rigorous, one should introduce a regularization of $\varepsilon(x)$ of the form $\varepsilon_{p}(\rho(x))$, so that its gradient is proportional to $n \circ \pi(x)$ and let $p$ go to $+\infty$ in the relation $\left.\nabla\left(\varepsilon_{p} \circ \rho\right) \cdot \nabla \bar{\psi}_{i}^{2}=0\right)$. Hence,

$$
\begin{aligned}
\tilde{\mathcal{L}} \tilde{u}(x)= & \nabla \cdot(n \circ \pi) \circ\left(\bar{\psi}_{i}\right)^{-1}(x) \frac{\partial \tilde{u}}{\partial x_{1}}(x)+\varepsilon^{\#}\left(x_{1}\right) \Delta \bar{\psi}_{i}^{2} \circ\left(\bar{\psi}_{i}\right)^{-1}(x) \frac{\partial \tilde{u}}{\partial x_{2}}(x) \\
& +\frac{1}{\varepsilon^{\#}\left(x_{1}\right)} \frac{\partial^{2} \tilde{u}}{\partial x_{1}^{2}}(x)+\varepsilon^{\#}\left(x_{1}\right)\left\|\nabla \bar{\psi}_{i}^{2} \circ\left(\bar{\psi}_{i}\right)^{-1}(x)\right\|^{2} \frac{\partial^{2} \tilde{u}}{\partial x_{2}^{2}}(x) .
\end{aligned}
$$

Now, set $\zeta_{h}(x):=h^{-2} \zeta(x / h)$ where $\zeta(x)$ is a $C^{\infty}$ non-negative function with compact support in $\mathbb{R}^{2}$ and with $L^{1}$ norm equal to 1 . Set $\tilde{u}^{(n)}(x):=\tilde{u} * \zeta_{1 / n}$ and $u^{(n)}=\tilde{u}^{(n)} \circ \bar{\psi}_{i}$ for all $n \geq 1$. The function $u^{(n)}$ obviously satisfies (2.12) and (2.32). Since moreover

$$
\nabla^{\mathrm{int}} u^{(n)}(x) \cdot n(x)=\left(\nabla \tilde{u}^{(n)}\left(\bar{\psi}_{i}(x)\right)\right)^{\prime} J^{\mathrm{int}} \bar{\psi}_{i}(x) n(x)=\frac{1}{\varepsilon_{\mathrm{int}}} \frac{\partial \tilde{u}^{(n)}}{\partial x_{1}}(\bar{\psi}(x)), \quad \forall x \in \Gamma
$$

$u^{(n)}$ also satisfies (2.31). Therefore, (2.34) holds for $u^{(n)}$.

Since $\tilde{u}$ is continuous, $\tilde{u}^{(n)}$ converges to $\tilde{u}$ in $L^{\infty}\left(\mathbb{R}^{d}\right)$ when $n \rightarrow+\infty$. Similarly, since $\nabla \tilde{u}$ and $\nabla^{2} \tilde{u}$ are continuous and bounded on $(\mathbb{R} \backslash\{0\}) \times \mathbb{R}, \tilde{\mathcal{L}} \tilde{u}^{(n)}$ (resp. $\nabla \tilde{u}^{(n)}$ ) converges to $\tilde{\mathcal{L}} \tilde{u}$ (resp. $\nabla \tilde{u}$ ) in the bounded pointwise sense in $(\mathbb{R} \backslash\{0\}) \times \mathbb{R}$. Since $\bar{\psi}_{i}$ is continuous, we have proved that $u^{(n)}\left(\operatorname{resp} . \mathcal{L} u^{(n)}\right.$ and $\left.\nabla u^{(n)}\right)$ converges in $L^{\infty}\left(\mathbb{R}^{2}\right.$ ) (resp. in the bounded pointwise sense in $\mathbb{R}^{2} \backslash \Gamma$ ) to $u$ (resp. $\mathcal{L} u$ and $\nabla u$ ). Moreover, by (2.16), the functions $\mathcal{L} u^{(n)}$ are uniformly bounded in $L^{\infty}\left(\mathbb{R}^{2}\right)$. 
As a consequence, $u^{(n)}\left(X_{t}\right)-u^{(n)}\left(X_{0}\right)$ converges a.s. for all $t \geq 0$ to $u\left(X_{t}\right)-u\left(X_{0}\right)$. Similarly, by Lebesgue's theorem, $\int_{0}^{t} \mathbb{1}_{\left\{X_{s} \notin \Gamma\right\}} \mathcal{L} u^{(n)}\left(X_{s}\right) \mathrm{d} s$ converges a.s. for all $t \geq 0$ to $\int_{0}^{t} \mathbb{1}_{\left\{X_{s} \notin \Gamma\right\}} \mathcal{L} u\left(X_{s}\right) \mathrm{d} s$. Now, define the processes

$$
M_{t}^{(n)}=\int_{0}^{t} \sqrt{2 \varepsilon\left(X_{s}\right)} \nabla^{\mathrm{int}} u^{(n)}\left(X_{s}\right) \cdot \mathrm{d} B_{s} \quad \text { and } \quad M_{t}=\int_{0}^{t} \mathbb{1}_{\left\{X_{s} \notin \Gamma\right\}} \sqrt{2 \varepsilon\left(X_{s}\right)} \nabla u\left(X_{s}\right) \cdot \mathrm{d} B_{s} .
$$

The process $M^{(n)}-M$ is a $L^{2}$ martingale, whose quadratic variation is given by

$$
\left\langle M^{(n)}-M\right\rangle_{t}=2 \int_{0}^{t} \mathbb{1}_{\left\{X_{s} \in \Gamma\right\}} \varepsilon\left(X_{s}\right)\left\|\nabla^{\mathrm{int}} u^{(n)}\left(X_{s}\right)\right\|^{2} \mathrm{~d} s+2 \int_{0}^{t} \mathbb{1}_{\left\{X_{s} \notin \Gamma\right\}} \varepsilon\left(X_{s}\right)\left\|\nabla u^{(n)}\left(X_{s}\right)-\nabla u\left(X_{s}\right)\right\|^{2} \mathrm{~d} s .
$$

The first term of the right hand side is a.s. zero for all $t \geq 0$ using the occupation time formula similarly as in the end of the proof of Lemma 2.7. By Lebesgue's theorem again, the second term of the right hand side a.s. converges to 0 for all $t \geq 0$, and is uniformly bounded on finite time intervals. Therefore, by Doob's inequality, for all $T>0$ and $\eta>0$,

$$
\mathbb{P}\left(\sup _{t \in[0, T]}\left|M_{t}^{(n)}-M_{t}\right| \geq \eta\right) \leq C \frac{\mathbb{E}\left(\left\langle M^{(n)}-M\right\rangle_{T}\right)}{\eta^{2}} .
$$

Hence, by Lebesgue's theorem again, $M_{t}^{(n)}$ converges to $M_{t}$ in probability for the $L^{\infty}([0, T])$ norm. Consequently, (2.30) follows from (2.34) by letting $n$ go to infinity, which completes the proof of Corollary 2.9.

\subsection{Equivalence between the martingale problem of Definition 2.2 and weak solutions}

The aim of the next section is to prove the uniqueness of the solution to the martingale problem of Definition 2.2. A key step is to show that solving this problem is equivalent to exhibiting a weak solution. One implication in this equivalence is an immediate consequence of Corollary 2.9.

Theorem 2.10. Assume that $\Gamma$ is a compact $C^{3}$ submanifold of $\mathbb{R}^{d}$. Then, for any $x \in \mathbb{R}^{d}$, the law $\mathbb{P}_{x}$ of the process $X$ constructed in the proof of Theorem 2.4 started at $x$, satisfies (2.11)-(2.14).

In order to show that a solution to the above martingale problem provides the existence of a weak solution to (2.17), we need the following lemma.

Lemma 2.11. Fix $\mathbb{P}$ a probability measure on $\left(\mathcal{C}, \mathcal{B}_{t}\right)$ satisfying $(2.14)$. Define $i_{1}$ as in $(2.21)$ and let

$$
\tau_{1}=\inf \left\{t \geq 0: w(t) \notin \mathcal{U}_{i_{1}}\right\} \wedge 1
$$

Then, one can construct on the space $\left(\mathcal{C},\left(\mathcal{B}_{t}\right), \mathbb{P}\right)$ a Brownian motion $B$ on the time interval $\left[0, \tau_{1}\right]$ such that the canonical process $(w(t))$ is $\mathbb{P}$-a.s. solution to (2.17) for this Brownian motion on the time interval $\left[0, \tau_{1}\right]$.

Proof. Note that nothing is assumed on the law of $w(0)$ under $\mathbb{P}$. In particular, the integer

$$
i_{1}:=\min \left\{i \in\{1, \ldots, M\}: \mathrm{d}\left(w(0), \mathcal{U}_{i}^{c}\right)=\max _{1 \leq j \leq M} \mathrm{~d}\left(w(0), \mathcal{U}_{j}^{c}\right)\right\}
$$

may be random.

Fix $1 \leq i \leq M$ and assume first that $i_{1}=i \mathbb{P}$-a.s. As in the proof of Theorem 2.4, assume that $d=2$ for simplicity. Let us extend arbitrarily the functions $\nabla \cdot(n \circ \pi) \circ\left(\bar{\psi}_{i}\right)^{-1}, \Delta \bar{\psi}_{i}^{2} \circ\left(\bar{\psi}_{i}\right)^{-1}$ and $\nabla \bar{\psi}_{i}^{2} \circ\left(\bar{\psi}_{i}\right)^{-1}$ out of $\mathcal{V}_{i}$ as bounded functions. Then, we may extend the definition of $\tilde{\mathcal{L}} \tilde{\varphi}$ to any $C^{2}\left(\mathbb{R}^{2}\right)$ function $\tilde{\varphi}$ by the formula (2.35) where the coefficients are replaced by their extensions. Now, for any $\tilde{\varphi} \in C^{2}\left(\mathbb{R}^{2}\right)$, and for any function $\chi \in C_{c}^{2}\left(\mathcal{V}_{i}\right)$, the function defined by $\chi\left(\bar{\psi}_{i}(x)\right) \tilde{\varphi}\left(\bar{\psi}_{i}(x)\right.$ ) for all $x \in \mathbb{R}^{2}$ (with the convention that 
this quantity is 0 if $x \notin \mathcal{U}_{i}$ ) satisfies (2.12) and (2.13). Therefore, (2.14) holds for this function, or, equivalently, setting $Z_{t}=\bar{\psi}_{i}\left(w\left(t \wedge \tau_{1}\right)\right)$,

$$
\tilde{\varphi}\left(Z_{t \wedge \tau_{1}}\right)-\tilde{\varphi}\left(Z_{0}\right)-\int_{0}^{t \wedge \tau_{1}} \tilde{\mathcal{L}} \tilde{\varphi}\left(Z_{s}\right) \mathrm{d} s
$$

is a $\mathbb{P}$-martingale, where $\tau_{1}:=\inf \left\{t \geq 0: Z_{t} \in \partial \mathcal{V}_{i}\right\} \wedge 1$.

The operator $\tilde{\mathcal{L}}$ is a second-order differential operator with Borel bounded coefficients, so it satisfies the assumptions of the classical martingale problems theory. In particular, using standard techniques (see the proof of Thm. 20.1 in Chap. V of [29] and particularly equation (20.5)), a two-dimensional Brownian motion $W$ can be constructed on $\left(\mathcal{C}, \mathcal{B}_{t}, \mathbb{P}\right)$ on the time interval $\left[0, \tau_{1}\right]$ such that $\left(Z_{t}\right)$ solves the (classical) SDE

$$
\begin{aligned}
\mathrm{d} Z_{t}^{1} & =\sqrt{\frac{2}{\varepsilon^{\#}\left(Z_{t}^{1}\right)}} \mathrm{d} W_{t}^{1}+\Delta \rho \circ\left(\bar{\psi}_{i}\right)^{-1}\left(Z_{t}\right) \mathrm{d} t, \\
\mathrm{~d} Z_{t}^{2} & =\sqrt{2 \varepsilon^{\#}\left(Z_{t}^{1}\right)}\left\|\nabla \bar{\psi}_{i}^{2} \circ\left(\bar{\psi}_{i}\right)^{-1}\left(Z_{t}\right)\right\| \mathrm{d} W_{t}^{2}+\varepsilon^{\#}\left(Z_{t}^{1}\right) \Delta \bar{\psi}_{i}^{2} \circ\left(\bar{\psi}_{i}\right)^{-1}\left(Z_{t}\right) \mathrm{d} t,
\end{aligned}
$$

on $\left[0, \tau_{1}\right]$. (It is not necessary to enlarge the probability space $(\mathcal{C}, \mathcal{B}, \mathbb{P})$ since the diffusion matrix is uniformly non-degenerate.) Thus, by Itô's formula, there exists a $\left(\mathbb{P}, \mathcal{B}_{t}\right)$-Brownian motion $B$ on $\left[0, \tau_{1}\right]$, explicitly expressed in terms of $Z_{t}$ and $W_{t}$, such that $\bar{\psi}^{-1}\left(Z_{t}\right)=w(t)$ solves (2.17), as required.

Now, take an arbitrary $\mathbb{P}$ satisfying the assumptions of Lemma 2.11, under which $i_{1}$ is not necessarily a.s. constant. For all $1 \leq i \leq M$ such that $\mathbb{P}\left(i_{1}=i\right)>0$, the previous construction can be applied to the probability measure $\mathbb{P}^{i}=\mathbb{P}\left(\cdot \mid i_{1}=i\right)$, giving a $\mathbb{P}^{i}$-Brownian motion $B^{(i)}$ on $\left[0, \tau_{1}\right]$, on the space $\mathcal{C}$. Setting $B_{t}^{(j)}=0$ for all $t \geq 0$ if $\mathbb{P}\left(i_{1}=j\right)=0$, we can define the process

$$
B_{t}(w)=\sum_{i=1}^{M} \mathbb{1}_{\left\{i_{1}(w)=i\right\}} B_{t}^{i}(w) .
$$

It is then easy to check from Lévy's theorem that this is a $\mathbb{P}$-Brownian motion on $\left[0, \tau_{1}\right]$. Moreover, it obviously satisfies the statement of Lemma 2.11.

Theorem 2.12. Assume that $\Gamma$ is a compact $C^{3}$ submanifold of $\mathbb{R}^{d}$. If $\mathbb{P}_{x}$ is a probability measure on $(\mathcal{C}, \mathcal{B})$ satisfying (2.11)-(2.14) for some $x \in \mathbb{R}^{d}$, then there exists a $\mathbb{P}_{x}-\left(\mathcal{B}_{t}\right)$ Brownian motion $B$ on $\mathcal{C}$ such that the canonical process $(w(t), t \geq 0)$ solves $(2.17) \mathbb{P}_{x}$-a.s. In particular, $w(0)=x \mathbb{P}_{x}$-a.s.

Proof. Let us define on the space $\left(\mathcal{C},\left(\mathcal{B}_{t}\right)_{t \geq 0}, \mathcal{B}\right)$ sequences of random integers $\left(i_{k}\right)_{k \geq 1}$ and of stopping times $\left(\tau_{k}\right)_{k \geq 1}$ similarly as in the proof of Theorem 2.4 , by substituting $(w(t))$ to $\left(X_{t}\right)$.

For all $k \geq 1$ and for all $A \in \mathcal{B}$, define

$$
\mathbb{P}^{(k)}(A)=\mathbb{P}_{x} \circ \theta_{\tau_{k}}^{-1}(A)=\mathbb{P}_{x}\left\{w \in \mathcal{C}: w^{(k)} \in A\right\}
$$

where $w^{(k)}(t)=w \circ \theta_{\tau_{k}(w)}(t)=w\left(t+\tau_{k}(w)\right)$ for all $t \geq 0$. For all $k \geq 1, \mathbb{P}^{(k)}$ is a probability measure on $(\mathcal{C}, \mathcal{B})$. Moreover, for all $t \geq s \geq 0, A \in \mathcal{B}_{s}$, and $\varphi$ satisfying (2.12) and (2.13), defining $A^{\prime}=\left\{w \in \mathcal{C}: w^{(k)} \in A\right\}$, we have

$$
\mathbb{E}^{(k)}\left[\left(M_{t}^{\varphi}-M_{s}^{\varphi}\right) \mathbb{1}_{A}\right]=\mathbb{E}\left[\left(M_{t}^{\varphi}\left(w^{(k)}\right)-M_{s}^{\varphi}\left(w^{(k)}\right)\right) \mathbb{1}_{A}\left(w^{(k)}\right)\right]=\mathbb{E}\left[\left(M_{\tau_{k}+t}^{\varphi}-M_{\tau_{k}+s}^{\varphi}\right) \mathbb{1}_{A^{\prime}}\right]=0
$$

Hence, $\mathbb{P}^{(k)}$ satisfies $(2.14)$ and Lemma 2.11 applies. This provides a $\left(\mathcal{C}, \mathcal{B}_{t}, \mathbb{P}^{(k)}\right)$-Brownian motion $\left(B_{t}^{(k)}\right.$, $\left.0 \leq t \leq \tau_{1}\right)$. 
The Brownian motion $B$ of Theorem 2.12 can be constructed as follows: observe first that $\tau_{\infty}:=\sup _{k>1} \tau_{k}(w)=$ $+\infty$ for all $w \in \mathcal{C}$, since otherwise, $w$ would admit no left limit at time $\tau_{\infty}$. Now, for all $t \geq 0$ and $w \in \mathcal{C}$, define

$$
B_{t}(w)=\sum_{k=0}^{+\infty} B_{t \wedge \tau_{k+1}(w)-t \wedge \tau_{k}(w)}^{(k+1)}\left(w^{(k)}\right)
$$

where $\tau_{0}=0$. The fact that the process $w \mathbb{P}$-a.s. solves $(2.17)$ driven by the process $B$ on each time interval $\left[\tau_{k}, \tau_{k+1}\right), k \geq 0$, follows from the definition of $\mathbb{P}^{(k)}$. Hence, it only remains to check that $B$ is a $\mathbb{P}_{x}$-Brownian motion.

For all $k \geq 0$, let

and define the time-shifted $\sigma$-field

$$
M_{t}^{(k)}=B_{t \wedge \tau_{k+1}(w)-t \wedge \tau_{k}(w)}^{(k+1)}\left(w^{(k)}\right)
$$

$$
\mathcal{G}^{(k)}=\left\{\left\{w \in \mathcal{C}: w^{(k)} \in B\right\}: B \in \mathcal{B}\right\}
$$

First, let us prove that $B$ is a $\left(\mathcal{B}_{t}\right)$ martingale. Fix $t \geq s \geq 0$. For all $A \in \mathcal{B}_{\tau_{k}+s}$,

$$
\mathbb{E}\left[\left(M_{\tau_{k}+t}^{(k)}-M_{\tau_{k}+s}^{(k)}\right) \mathbb{1}_{A}\right]=\mathbb{E}\left[\left(M_{\tau_{k}+t}^{(k)}-M_{\tau_{k}+s}^{(k)}\right) \mathbb{P}\left[A \mid \mathcal{G}^{(k)}\right]\right]=\mathbb{E}^{(k)}\left[\left(B_{t \wedge \tau_{1}}^{(k+1)}-B_{s \wedge \tau_{1}}^{(k+1)}\right) A^{\prime}\right]=0
$$

where $A^{\prime}$ is the random variable on $\mathcal{C}$ defined by $\mathbb{P}\left(A \mid \mathcal{G}^{(k)}\right)(w)=A^{\prime}\left(w^{(k)}\right)$ a.s. Therefore, for all $0 \leq s \leq t$,

$$
\mathbb{E}\left[M_{\tau_{k}+t}^{(k)} \mid \mathcal{B}_{\tau_{k}+s}\right]=M_{\tau_{k}+s}^{(k)} \quad \text { a.s. }
$$

From this can be classically deduced that, for all a.s. finite stopping times $T \geq S \geq \tau_{k}$,

$$
\mathbb{E}\left[M_{T}^{(k)} \mid \mathcal{B}_{S}\right]=M_{S}^{(k)} \text { a.s. }
$$

Finally, observing that $M_{t}^{(k)}=0$ if $t \leq \tau_{k}$, for any a.s. finite stopping times $0 \leq S \leq T$,

$$
\mathbb{E}\left[M_{T}^{(k)} \mid \mathcal{B}_{S}\right]=\mathbb{E}\left[\mathbb{E}\left[M_{T \vee \tau_{k}}^{(k)} \mid \mathcal{B}_{S \vee \tau_{k}}\right] \mid \mathcal{B}_{S}\right]=\mathbb{E}\left[M_{S \vee \tau_{k}}^{(k)} \mid \mathcal{B}_{S}\right]=M_{S}^{(k)}
$$

Therefore $M^{(k)}$ is a $\left(\mathcal{B}_{t}\right)$ martingale for all $k \geq 0$. The fact that $\left(B_{t}, t \geq 0\right)$ is a $\left(\mathcal{B}_{t}\right)$ martingale then follows.

Using similar computations, one can also deduce from the fact that $\left\langle B^{(k)}\right\rangle_{t}=\left(t \wedge \tau_{1}\right)$ Id, where Id is the $d$-dimensional identity matrix, that

$$
\left(M_{t}^{(k)}\right)^{T} M_{t}^{(k)}-\left(t \wedge \tau_{k+1}-\tau_{k}\right)_{+} \mathrm{Id}
$$

is a $\left(\mathcal{B}_{t}\right)$ martingale for all $k \geq 1$, where $u^{T}$ denotes the transpose of the vector $u \in \mathbb{R}^{d}$. Moreover, for all $0 \leq i<j$ and $0 \leq s \leq t$, since $M^{(i)}$ is constant after time $\tau_{i+1}$ and $M^{(j)}$ is 0 before time $\tau_{i+1}$,

$$
\mathbb{E}\left[\left(M_{t}^{(i)}\right)^{T} M_{t}^{(j)} \mid \mathcal{B}_{s}\right]=\mathbb{E}\left[\left(M_{\tau_{i+1} \vee s}^{(i)}\right)^{T} \mathbb{E}\left[M_{t}^{(j)} \mid \mathcal{B}_{\tau_{i+1} \vee s}\right] \mid \mathcal{B}_{s}\right]=\mathbb{E}\left[\left(M_{\tau_{i+1} \vee s}^{(i)}\right)^{T} M_{s}^{(j)} \mid \mathcal{B}_{s}\right]=\left(M_{s}^{(i)}\right)^{T} M_{s}^{(j)}
$$


Combining the above results, we obtain that for all $k \geq 1$ and $0 \leq s \leq t$,

$$
\begin{aligned}
\mathbb{E}\left[B_{t \wedge \tau_{k}}^{T} B_{t \wedge \tau_{k}} \mid \mathcal{B}_{s}\right] & =\sum_{i=0}^{k-1}\left[\left(M_{s}^{(i)}\right)^{T} M_{s}^{(i)}+\left(t \wedge \tau_{i+1}-\tau_{i}\right)_{+} \mathrm{Id}-\left(s \wedge \tau_{i+1}-\tau_{i}\right)_{+} \mathrm{Id}\right]+2 \sum_{0 \leq i<j \leq k-1}\left(M_{s}^{(i)}\right)^{T} M_{s}^{(j)} \\
& =B_{s \wedge \tau_{k}}^{T} B_{s \wedge \tau_{k}}+\left(t \wedge \tau_{k}-s \wedge \tau_{k}\right) \mathrm{Id} .
\end{aligned}
$$

In other words, $\langle B\rangle_{t}=t$ Id for all $t \geq 0$, which concludes the proof by Lévy's theorem.

\subsection{Uniqueness and strong Markov property of the solution of the martingale problem of Definition 2.2}

We now are in a position to prove the uniqueness of the solution of the martingale problem of Definition 2.2 (or, equivalently, the uniqueness in the sense of probability law of the weak solution to the SDE (2.17)). We start with the following observation.

Remark 2.13. In the proof of Theorem 2.12, the construction of the Brownian motion $B$ uses the martingale property (2.14) for countably many functions. More precisely, fix $1 \leq i \leq M$ and let $\left(\mathcal{U}_{i}^{(p)}\right)_{p \geq 1}$ be a sequence of compact subsets of $\mathcal{U}_{i}$ such that $\mathbb{1}_{\mathcal{U}_{i}^{(p)}}$ converges to $\mathbb{1}_{\mathcal{U}_{i}}$ everywhere in $\mathbb{R}^{d}$ and such that all $x \in \mathcal{U}_{i}^{(p)}$ if $\mathrm{d}\left(x, \mathcal{U}_{i}^{c}\right)>1 / p$ and $|x| \leq p$. Then, the Theorem 20.1 in [29] which we use in the proof of Theorem 2.12 only requires that (2.14) holds for a function satisfying (2.12) and (2.13), with compact support in $\mathcal{U}_{i}$ and equal to $\bar{\psi}_{i}^{j}(x)$ in $\mathcal{U}_{i}^{(p)}$, and similarly for the function $\bar{\psi}_{i}^{j}(x) \bar{\psi}_{i}^{k}(x)$, for all $p \geq 1, j, k \in\{1, \ldots, d\}$ and $i \in\{1, \ldots, M\}$. This observation will be useful in the proof of the following result.

Theorem 2.14. Assume that $\Gamma$ is a compact $C^{3}$ submanifold of $\mathbb{R}^{d}$. Then, the martingale problem (2.11)-(2.14) is well-posed, there is a unique weak solution to the SDE (2.17) in the sense of the probability law, and the family of solutions constructed in Theorem 2.12 is strong Markov.

Proof. Let $\mathbb{P}_{x}^{1}$ and $\mathbb{P}_{x}^{2}$ be two probability measures on $(\mathcal{C}, \mathcal{B})$ satisfying (2.11) and (2.14). Using the notation of the proof of Theorem 2.12, one can construct on $\left(\mathcal{C}, \mathcal{B},\left(\mathcal{B}_{t}\right)\right)$ the sequences $\left(i_{k}\right)_{k \geq 1}$ and $\left(\tau_{k}\right)_{k \geq 1}$, a $\left(\mathbb{P}_{x}^{1}, \mathcal{B}_{t}\right)$ Brownian motion $B^{1}$, a $\left(\mathbb{P}_{x}^{2}, \mathcal{B}_{t}\right)$ Brownian motion $B^{2}$, such that $\mathbb{P}_{x}^{1}$-a.s. (respectively, $\mathbb{P}_{x}^{2}$-a.s.) the canonical process $w$ on $\mathcal{C}$ solves the $\operatorname{SDE}(2.17)$ driven by $B^{1}$ (respectively, $B^{2}$ ).

The integer $i_{1}$ only depends on $x$. Therefore, assuming for simplicity that $d=2$ and using the notation of the proof of Lemma 2.11, under $\mathbb{P}_{x}^{k}(k=1$ or 2$)$, on the event $\left\{i_{1}=i\right\}$ for $1 \leq i \leq M-1, \bar{\psi}_{i_{1}}(w(t))$ solves $(2.36)-(2.37)$ on the time interval $\left[0, \tau_{1}\right]$, where $W_{t}^{j}=\int_{0}^{t \wedge \tau_{1}} \nabla \bar{\psi}_{i}^{j} \circ\left(\bar{\psi}_{i_{1}}\right)^{-1}\left(\xi_{t}\right) \cdot \mathrm{d} B_{t}^{k}$ for $j=1,2$. From these expressions, we deduce from (2.1) and (2.3) that $W^{1}$ and $W^{2}$ are independent Brownian motions on the time interval $\left[0, \tau_{1}\right]$. Now, using Girsanov's theorem, one can construct from $\left.\mathbb{P}_{x}^{1}\right|_{\mathcal{F}_{\tau_{1}}}$ (resp. $\left.\mathbb{P}_{x}^{2}\right|_{\mathcal{F}_{\tau_{1}}}$ ) an equivalent probability measure $\mathbb{Q}^{1}$ (resp. $\mathbb{Q}^{2}$ ) on $\mathcal{F}_{\tau_{1}}$ under which, on the event $\left\{i_{1}=i\right\}, \bar{\psi}_{i_{1}}(w(t))$ solves

$$
\begin{aligned}
\mathrm{d} Z_{t}^{1} & =\sqrt{\frac{2}{\varepsilon^{\#}\left(Z_{t}^{1}\right)}} \mathrm{d} \tilde{W}_{t}^{1}, \\
\mathrm{~d} Z_{t}^{2} & =\sqrt{2 \varepsilon^{\#}\left(Z_{t}^{1}\right)}\left\|\nabla \bar{\psi}_{i}^{2} \circ\left(\bar{\psi}_{i}\right)^{-1}\left(Z_{t}\right)\right\|_{2} \mathrm{~d} \tilde{W}_{t}^{2},
\end{aligned}
$$

on the time interval $\left[0, \tau_{1}\right]$, where $\left(\tilde{W}_{t}^{1}, \tilde{W}_{t}^{2}\right)$ is a $\mathbb{Q}^{1}$-Brownian motion (resp. a $\mathbb{Q}^{2}$-Brownian motion) on the time interval $\left[0, \tau_{1}\right]$. Now, similarly as in the proof of Theorem 2.4 , since the first equation is closed and the diffusion coefficient of the second equation has a Lipschitz dependence w.r.t. $Z_{t}^{2}$, there is pathwise uniqueness for this SDE. Hence, $\mathbb{Q}^{1}=\mathbb{Q}^{2}$, which implies by the Girsanov theorem that $\left.\mathbb{P}_{x}^{1}\right|_{\mathcal{B}_{\tau_{1}}}=\left.\mathbb{P}_{x}^{2}\right|_{\mathcal{B}_{\tau_{1}}}$ (see $[29]$, Thm. (27.1)).

In particular, the laws of $w\left(\tau_{1}(w)\right)$, and thus those of $i_{2}$, are the same under $\mathbb{P}_{x}^{1}$ and $\mathbb{P}_{x}^{2}$. Therefore, proceeding as before, $\left.\mathbb{P}_{x}^{1} \circ \theta_{\tau_{1}}^{-1}\right|_{\mathcal{B}_{\tau_{1}}}=\left.\mathbb{P}_{x}^{2} \circ \theta_{\tau_{1}}^{-1}\right|_{\mathcal{B}_{\tau_{1}}}$ or, equivalently, $\left.\mathbb{P}_{x}^{1}\right|_{\mathcal{G}^{(1)} \cap \mathcal{B}_{\tau_{2}}}=\left.\mathbb{P}_{x}^{2}\right|_{\mathcal{G}^{(1)} \cap \mathcal{B}_{\tau_{2}}}$, where $\mathcal{G}^{(k)}$ is defined in (2.39). 
By induction, we obtain that

$$
\left.\mathbb{P}_{x}^{1}\right|_{\mathcal{G}^{(k-1)} \cap \mathcal{B}_{\tau_{k}}}=\left.\mathbb{P}_{x}^{2}\right|_{\mathcal{G}^{(k-1)} \cap \mathcal{B}_{\tau_{k}}} \quad \forall k \geq 1
$$

with the convention that $\mathcal{G}^{(0)}=\mathcal{B}$.

The well-posedness of the martingale problem then results from the equality

$$
\sigma\left(\bigcup_{k \geq 1}\left(\mathcal{G}^{(k-1)} \cap \mathcal{B}_{\tau_{k}}\right)\right)=\mathcal{B},
$$

which can be proved as follows: for all Borel subset $A$ of $\mathbb{R}^{d}$ and all $t \geq 0$, the continuity of the paths of $w$ implies that

$$
\begin{aligned}
\{w(t) \in A\} & \cap\left\{t \leq \tau_{2}\right\}=\left\{w(t) \in A, t \leq \tau_{1}\right\} \\
& \bigcup \lim _{n \rightarrow \infty} \bigcup_{m \geq 1}\left(\left\{\tau_{1} \in\left[t-m / 2^{n}, t-(m-1) / 2^{n}\right)\right\} \bigcap\left\{w^{(1)}\left((m-1) / 2^{n}\right) \in A, \tau_{2}-\tau_{1} \geq(m-1) / 2^{n}\right\}\right) .
\end{aligned}
$$

The first event of the right-hand side belongs to $\mathcal{G}^{(0)} \cap \mathcal{B}_{\tau_{1}}=\mathcal{B}_{\tau_{1}}$, and for all $n, m \geq 1$, the event $\left\{\tau_{1} \in\right.$ $\left.\left[t-m / 2^{n}, t-(m-1) / 2^{n}\right)\right\}$ belongs to $\mathcal{B}_{\tau_{1}}$ and the event $\left\{w^{(1)}\left((m-1) / 2^{n}\right) \in A, \tau_{2}-\tau_{1} \geq(m-1) / 2^{n}\right\}$ belongs to $\mathcal{G}^{(1)} \cap \mathcal{B}_{\tau_{2}}$. Hence,

$$
\{w(t) \in A\} \cap\left\{t \leq \tau_{2}\right\} \in \sigma\left(\mathcal{B}_{\tau_{1}} \cup\left(\mathcal{G}^{(1)} \cap \mathcal{B}_{\tau_{2}}\right)\right)
$$

Similarly, we have that, for all $k \geq 1$,

$$
\{w(t) \in A\} \cap\left\{t \leq \tau_{k}\right\} \in \sigma\left(\bigcup_{l=1}^{k}\left(\mathcal{G}^{(l-1)} \cap \mathcal{B}_{\tau_{l}}\right)\right)
$$

and hence, using the fact that $\tau_{\infty}(w)=+\infty$ for all $w \in \mathcal{C}$,

$$
\{w(t) \in A\} \in \sigma\left(\bigcup_{k \geq 1}\left(\mathcal{G}^{(k-1)} \cap \mathcal{B}_{\tau_{k}}\right)\right) .
$$

A similar construction can be done for any finite dimensional cylindrical measurable set on $\mathcal{C}$, which ends the proof of the uniqueness in law for the SDE (2.17).

As in the case of classical martingale problems for elliptic operators with bounded coefficients, the strong Markov property follows from the well-posedness of the martingale problem. The proof of Theorem 4.5.1 of [10] adapts straightforwardly to our case. Note that this proof makes use of Remark 2.13, as explained in the proof of Theorem 5.4.20 and in Remark 5.4.12 of [11].

The strong Markov property allows us to prove the following path property of the weak solution $X$ which we will use to establish the Feynman-Kac formula in Section 2.7.

Proposition 2.15. Assume that $\Gamma$ is a $C^{3}$ compact submanifold of $\mathbb{R}^{d}$ and let $K$ be a compact set. Then, the time spent in $\mathbb{R}^{d} \backslash K$ by the weak solution to (2.17) is a.s. infinite. We also have

$$
\limsup _{t \rightarrow+\infty}\left|X_{t}\right|=+\infty \quad \text { a.s. }
$$

and, if $d \geq 3$,

$$
\lim _{t \rightarrow+\infty}\left|X_{t}\right|=+\infty \quad \text { a.s. }
$$


Proof. Let us first prove that (2.41) holds for any dimension $d$. Fix $x \in \mathbb{R}^{d}$. The classical Aronson's estimates [1] entails that the law of $X_{t}$ when $X_{0}=x$ has a density $p(t, x, y)$ with respect to Lebesgue's measure which satisfies

$$
\frac{1}{C t^{d / 2}} \exp \left(-C|y-x|^{2} / t\right) \leq p(t, x, y) \leq \frac{M}{t^{d / 2}} \exp (-|x-y| / C t), \quad \forall t \geq 0, x, y \in \mathbb{R}^{d}
$$

(The study of Aronson's estimates in the context of stochastic processes can be found for example in [35].) From this easily follows that

$$
\lim _{t \rightarrow+\infty} \mathbb{P}_{x}\left(\left|X_{t}\right| \leq n\right)=0, \quad \forall n \geq 1 .
$$

Introducing an increasing sequence $\left(t_{m}\right)_{m \geq 1}$ converging to $+\infty$ and satisfying

$$
\mathbb{P}_{x}\left(\left|X_{t_{m}}\right| \leq n\right) \leq 2^{-m}, \quad \forall m \geq 1,
$$

Borel-Cantelli's lemma yields

$$
\mathbb{P}\left(\forall T>0, \exists t>T \text { s.t. }\left|X_{t}\right|>n\right)=1 .
$$

Since this holds for all $n \geq 1,(2.41)$ is proved.

This implies in particular that for all $n \geq 1$,

$$
\tau_{n}:=\inf \left\{t \geq 0:\left|X_{t}\right|=n\right\}
$$

is a.s. finite if $n \geq|x|$. Since $X_{t}$ has (scaled by $\sqrt{2 \varepsilon_{\text {ext }}}$ ) Brownian paths in $\Omega_{\text {ext }}$, the strong Markov property of Theorem 2.14 yields

$$
\lim _{n \rightarrow+\infty} \mathbb{P}_{x}\left(\exists t \in[0,1]: X_{\tau_{n}+t} \in K\right) \leq \lim _{n \rightarrow+\infty} \mathbb{P}\left(\exists t \in[0,1]:\left|W_{t}\right| \leq r / \sqrt{2 \varepsilon_{\text {ext }}}|| W_{0} \mid=n / \sqrt{2 \varepsilon_{\text {ext }}}\right)=0
$$

where $r>0$ is such that $K \subset B(0, r)$ and $\Gamma \subset B(0, r)$, and $W$ is a $d$-dimensional Brownian motion. A similar use of Borel-Cantelli's lemma as above implies that the time spent by $X_{t}$ out of $K$ is a.s. infinite.

Finally, for all $m \geq 1$, define

$$
L_{m}:=\sup \left\{t \geq 0:\left|X_{t}\right| \leq m\right\}
$$

which is infinite iff liminf $\left|X_{t}\right| \leq m$. The strong Markov property applied at time $\tau_{n}$ yields

$$
\mathbb{P}_{x}\left(L_{m}=\infty\right)=\mathbb{E}_{x}\left(\mathbb{P}_{X_{\tau_{n}}}\left(L_{m}=\infty\right)\right) \leq \mathbb{E}_{x}\left(\mathbb{P}_{X_{\tau_{n}}}\left(\tau_{m}<\infty\right)\right), \quad \forall m \geq 1
$$

Assuming $m$ large enough for $\Gamma \subset B(0, m)$ and using again that $X_{t}$ has scaled Brownian paths in $\Omega_{\text {ext }}$, one has

$$
\mathbb{P}_{x}\left(L_{m}=\infty\right) \leq \mathbb{P}\left(\exists t \geq 0:\left|W_{t}\right|=m / \sqrt{2 \varepsilon_{\mathrm{ext}}}|| W_{0} \mid=n / \sqrt{2 \varepsilon_{\mathrm{ext}}}\right)
$$

Since the r.h.s. converges to 0 when $n \rightarrow+\infty$ if $d \geq 3$ (cf. e.g. [28], Cor. (18.3), Chap. I), we have

$$
\mathbb{P}_{x}\left(L_{m}=\infty\right)=0, \quad \forall m \geq 1,
$$

which ends the proof of Proposition 2.15.

\subsection{An extended Feynman-Kac formula}

In this section we establish a probabilistic interpretation of linear elliptic PDEs driven by a divergence form operator (1.1) by means of the weak solution to the SDE (2.17).

Let $D$ be an open (possibly unbounded) connected domain in $\mathbb{R}^{d}$ and consider the PDE

$$
\begin{cases}-\nabla \cdot(\varepsilon \nabla u)(x)+\lambda(x) u(x)=g(x) & \text { for } x \in D \\ u(x)=h(x) & \text { for } x \in \partial D\end{cases}
$$

This PDE is similar to the Poisson-Boltzmann equation (1.2), except that the source term is not singular. 
We introduce the following assumptions:

(H1): The boundary $\partial D$ of $D$ satisfies an exterior sphere condition at every boundary point.

(H2): The function $h$ is bounded and continuous on $\partial D$.

(H3): The function $g$ has compact support in $\mathbb{R}^{d}$ and belongs to $C_{b}^{\infty}\left(\Omega_{\text {ext }} \cup \Gamma\right)$ and $C_{b}^{\infty}\left(\Omega_{\text {int }} \cup \Gamma\right)$.

(H4): The function $\lambda$ is bounded, non-negative in $\mathbb{R}^{d}$, belongs to $C_{b}^{\infty}\left(\Omega_{\text {ext }} \cup \Gamma\right)$ and $C_{b}^{\infty}\left(\Omega_{\text {int }} \cup \Gamma\right)$, and $\lambda(x) \geq \underline{\lambda}>0$ for $x$ out of a compact set.

Remark 2.16. Note that Assumptions (H3) and (H4) do not require the functions $g$ and $\lambda$ to be continuous on $\mathbb{R}^{d}$. They can be discontinuous on $\Gamma$, but their restrictions to $\Omega_{\text {int }}$ and $\Omega_{\text {ext }}$ can be extended to $\Gamma$ in a $C_{b}^{\infty}$ way. In particular, the function $\kappa(x)$ of the Poisson-Boltzmann equation (1.2) satisfies (H4).

We first need existence, uniqueness and regularity results for (2.42).

Theorem 2.17. Assume that $\Gamma$ is a $C^{\infty}$ compact submanifold of $\mathbb{R}^{d}$. Under Assumptions (H1)-(H4), (2.42) admits a unique solution $u$ in $H^{1}(D)$, which belongs to $C_{b}^{0}(\bar{D}) \cap C^{2}(D \backslash \Gamma)$ and such that $u_{\mid D \cap \Gamma} \in C^{\infty}(\Gamma \cap D)$. Moreover, letting $f$ be a $C^{2}$ function in $\bar{D}$ such that

$$
\forall x \in \mathcal{N}, \quad f(x)=\left(\frac{\varepsilon_{\text {int }}}{\varepsilon_{\text {ext }}}-1\right) \nabla^{\mathrm{int}} u(\pi(x)) \cdot n(\pi(x)),
$$

the function $\hat{u}$ defined in (2.26) belongs to $C^{2}(D \backslash \Gamma) \cap W_{\mathrm{loc}}^{2, \infty}(D)$.

Note that this result presumably holds true under weaker regularity conditions on $\Gamma$. For example, Theorem. 16.2 of Chapter 3 of [12] gives a $C^{1}$ regularity of $u$ in $\Omega_{\text {int }}$ and $\Omega_{\text {ext }}$ up to the boundary $\Gamma$, provided that $\Gamma$ is $C^{2}$. This suggests that one could hope to have a $C^{2}$ regularity for $u$ in $\Omega_{\text {int }}$ and $\Omega_{\text {ext }}$ up to $\Gamma$ provided that $\Gamma$ is $C^{3}$, and this would be enough to entail the conclusions of Theorem 2.17. However, we could not find such a result in [12] or elsewhere. Therefore, we choose for sake of completeness to give a full proof of Theorem 2.17 in Appendix A, based on classical energy computations for the PDEs obtained by the local straightenings we defined in Section 2.1.

We then have the following extension of Feynman-Kac's formula.

Theorem 2.18. Under the same assumptions as in Theorem 2.17, let $\tau$ be the first exit time of $X_{t}$ from $D$, and $\mathbb{P}_{x}$ the law of the solution $\left(X_{t}, t \geq 0\right)$ of the $S D E(2.17)$ with initial condition $X_{0}=x \in \mathbb{R}^{d}$. Then, for all $x \in \bar{D}$, the random variable

$$
\int_{0}^{\tau}\left|g\left(X_{t}\right)\right| \exp \left(-\int_{0}^{t} \lambda\left(X_{s}\right) \mathrm{d} s\right) \mathrm{d} t
$$

has finite expectation under $\mathbb{P}_{x}$ and

$$
u(x)=\mathbb{E}_{x}\left[h\left(X_{\tau}\right) \exp \left(-\int_{0}^{\tau} \lambda\left(X_{t}\right) \mathrm{d} t\right)+\int_{0}^{\tau} g\left(X_{t}\right) \exp \left(-\int_{0}^{t} \lambda\left(X_{s}\right) \mathrm{d} s\right) \mathrm{d} t\right] .
$$

Proof. In a first step, let us assume that $g \geq 0$.

Fix any bounded open set $D^{\prime}$ with $C^{\infty}$ boundary such that $D^{\prime} \subset D$ and $\mathrm{d}\left(D^{\prime}, \partial D\right)>0$, and let $v(x)=$ $\chi\left(\mathrm{d}\left(x, D^{\prime}\right)\right) u(x)$, where $\chi \in C_{b}^{\infty}(\mathbb{R}), \chi(y)=1$ for $y \leq 0$ and $\chi(y)=0$ for $y \geq \mathrm{d}\left(D^{\prime}, \partial D\right) / 2$. In particular, $v$ has compact support in $D$, is $C^{2}$ in $\Omega_{\text {ext }} \cup \Omega_{\text {int }}$ and satisfies $v(x)=u(x)$ for any $x \in D^{\prime}$. Applying Theorem 2.8 to $v$, taking as function $f$ the function $\chi\left(\mathrm{d}\left(x, D^{\prime}\right)\right) f(x)$, where $f$ is defined in (2.43), we obtain that, for all $t \leq \tau^{\prime}$ where $\tau^{\prime}$ is the first exit time of $X_{t}$ from $D^{\prime}$,

$$
u\left(X_{t}\right)=u\left(X_{0}\right)+\int_{0}^{t} \sqrt{2 \varepsilon\left(X_{s}\right)} \nabla^{\mathrm{int}} u\left(X_{s}\right) \cdot \mathrm{d} B_{s}+\int_{0}^{t} \varepsilon\left(X_{s}\right) \Delta u\left(X_{s}\right) \mathbb{1}_{\left\{X_{s} \notin \Gamma\right\}} \mathrm{d} s .
$$


Therefore, applying Itô's formula to $U_{t} \exp \left(V_{t}\right)$, where $U_{t}=u\left(X_{t}\right)$ and $V_{t}=\int_{0}^{t} \lambda\left(X_{s}\right) \mathrm{d} s$, we have for all $t \leq \tau^{\prime}$,

$$
\begin{aligned}
u\left(X_{t}\right) \mathrm{e}^{-\int_{0}^{t} \lambda\left(X_{s}\right) \mathrm{d} s}= & u\left(X_{0}\right)+\int_{0}^{t} \sqrt{2 \varepsilon\left(X_{s}\right)} \nabla^{\mathrm{int}} u\left(X_{s}\right) \mathrm{e}^{-\int_{0}^{s} \lambda\left(X_{\theta}\right) \mathrm{d} \theta} \cdot \mathrm{d} B_{s} \\
& +\int_{0}^{t}\left(\varepsilon\left(X_{s}\right) \Delta u\left(X_{s}\right)-\lambda\left(X_{t}\right) u\left(X_{t}\right)\right) \mathrm{e}^{-\int_{0}^{s} \lambda\left(X_{\theta}\right) \mathrm{d} \theta} \mathbb{1}_{\left\{X_{s} \notin \Gamma\right\}} \mathrm{d} s .
\end{aligned}
$$

In the preceding equation, we used the fact that $\int_{0}^{t} \lambda\left(X_{s}\right) \mathrm{d} s=\int_{0}^{t} \lambda\left(X_{s}\right) \mathbb{1}_{\left\{X_{s} \notin \Gamma\right\}} \mathrm{d} s$ a.s. for all $t \geq 0$, which can be proved using the occupation time formula as in the proof of Lemma 2.7 .

Now, $\tau^{\prime}<\infty$ a.s. by Proposition 2.15. Therefore, taking expectation with respect to $\mathbb{E}_{x}$, using the $u$ is strong solution to (2.42) in $D \backslash \Gamma$ and that $\nabla^{\text {int }} u$ is bounded in $D^{\prime}$, we get

$$
u(x)=\mathbb{E}_{x}\left[u\left(X_{\tau^{\prime}}\right) \mathrm{e}^{-\int_{0}^{\tau^{\prime}} \lambda\left(X_{t}\right) \mathrm{d} t}\right]+\mathbb{E}_{x}\left[\int_{0}^{\tau^{\prime}} g\left(X_{t}\right) \mathrm{e}^{-\int_{0}^{t} \lambda\left(X_{s}\right) \mathrm{d} s} \mathrm{~d} t\right] .
$$

By the monotone convergence theorem,

$$
\mathbb{E}_{x}\left[\int_{0}^{\tau^{\prime}} g\left(X_{t}\right) \mathrm{e}^{-\int_{0}^{t} \lambda\left(X_{s}\right) \mathrm{d} s} \mathrm{~d} t\right] \longrightarrow \mathbb{E}_{x}\left[\int_{0}^{\tau} g\left(X_{t}\right) \mathrm{e}^{-\int_{0}^{t} \lambda\left(X_{s}\right) \mathrm{d} s} \mathrm{~d} t\right] \in[0,+\infty]
$$

when $D^{\prime}$ converges to $D$. Moreover, since $u$ is bounded and continuous in $\bar{D}$, by Lebesgue's theorem,

$$
\mathbb{E}_{x}\left[\mathbb{1}_{\{\tau<\infty\}} u\left(X_{\tau^{\prime}}\right) \mathrm{e}^{-\int_{0}^{\tau^{\prime}} \lambda\left(X_{t}\right) \mathrm{d} t}\right] \longrightarrow \mathbb{E}_{x}\left[\mathbb{1}_{\{\tau<\infty\}} h\left(X_{\tau}\right) \mathrm{e}^{-\int_{0}^{\tau} \lambda\left(X_{t}\right) \mathrm{d} t}\right]
$$

when $D^{\prime}$ converges to $D$.

By Proposition 2.15 again, a.s. on the event $\{\tau=+\infty\}, X_{t}$ necessarily spends an infinite time in the region where $\lambda(\cdot) \geq \underline{\lambda}$ while staying inside $D$. Therefore, $\int_{0}^{\tau} \lambda\left(X_{t}\right) \mathrm{d} t=+\infty$ a.s. on $\{\tau=\infty\}$, and hence, by Lebesgue's theorem again,

when $D^{\prime}$ converges to $D$.

$$
\mathbb{E}_{x}\left[\mathbb{1}_{\{\tau=\infty\}} u\left(X_{\tau^{\prime}}\right) \mathrm{e}^{-\int_{0}^{\tau^{\prime}} \lambda\left(X_{t}\right) \mathrm{d} t}\right] \longrightarrow 0
$$

Finally, all the terms in the left and right hand sides of (2.46) converge to a limit as $D^{\prime} \rightarrow D$, and the limit of each term except the last one is finite. Therefore,

$$
\mathbb{E}_{x}\left[\int_{0}^{\tau} g\left(X_{t}\right) \mathrm{e}^{-\int_{0}^{t} \lambda\left(X_{s}\right) \mathrm{d} s} \mathrm{~d} t\right]<\infty
$$

ending the proof of Theorem 2.18 when $g \geq 0$.

For arbitrary $g$, we first use the previous result for a function $\tilde{g} \geq|g|$ satisfying the assumptions of Theorem 2.18. We deduce that the random variable (2.44) has finite expectation. Next we make the same computation as before to obtain (2.46). Letting $D^{\prime} \rightarrow D$, the convergence of the second term follows from Lebesgue's theorem.

\section{Probabilistic interpretation of Poisson-Boltzmann PDE and Monte Carlo algorithms}

The goal of this section is to apply the above theoretical results to the linearized Poisson-Boltzmann equation (1.2), in order to obtain probabilistic interpretations of its solution which are well-suited for Monte Carlo approximation methods. 
The numerical resolution of the PDE (1.2) by Monte Carlo methods is relevant in the biomolecular context for at least three reasons:

- In biology, this PDE is solved to compute the electrostatic free energy of the molecule, which is (proportional to) $\sum_{j=1}^{N} q_{j}\left(u-u_{0}\right)\left(x_{j}\right)$, where

$$
u_{0}(x)=\frac{1}{4 \pi \varepsilon_{\text {int }}} \sum_{l=1}^{N} \frac{q_{j}}{\left|x-x_{l}\right|} \quad \forall x \in \Omega_{\text {int }} .
$$

Therefore, the computation of the free energy only requires to compute $u_{0}$ at the $N$ points $x_{j}$.

- The problem is in dimension 3, and then Monte Carlo methods become competitive in comparison with deterministic methods.

- We take profit of the geometry of the molecule (that is, a union of spheres) to use efficient simulation methods of Brownian paths (see Sect. 3.2).

In all the sequel, $X$ denotes the solution of the $\operatorname{SDE}(2.17)$ and the dimension $d$ of the space is 3 .

\subsection{Probabilistic interpretations}

The PDE (1.2) fulfills all the assumptions of Theorem 2.17, except for the regularity of the source term $f$ in (1.4). We get rid of this problem as follows: $u_{0}$ defined in (3.1) is an explicit solution of

$$
\varepsilon_{\text {int }} \Delta u_{0}(x)=-f(x), \quad \forall x \in \Omega_{\text {int }} .
$$

Let $\chi$ be a $C^{\infty}$ function with compact support in $\Omega_{\text {int }}$ such that $\chi(x)=1$ for $x$ in a neighborhood of $\left\{x_{1}, \ldots, x_{N}\right\}$. Then, the function $v(x)=u(x)-\chi(x) u_{0}(x)$ solves the PDE

$$
-\nabla \cdot(\varepsilon(x) \nabla v(x))+\kappa^{2}(x) v(x)=\varepsilon_{\text {int }} u_{0}(x) \Delta \chi(x)+\varepsilon_{\text {int }} \nabla u_{0}(x) \cdot \nabla \chi(x)
$$

in $\mathbb{R}^{3}$. In other words, $v$ satisfies the same PDE as $u$ except that the singular source term is replaced by a $C^{\infty}$ source term with compact support. In particular, Theorem 2.17 applies to PDE (3.2), which provides our first probabilistic interpretation of the solution of Poisson-Boltzmann's equation. Note also that $u(x)-u_{0}(x)$ admits a finite limit when $x \rightarrow x_{i}$ for all $1 \leq i \leq N$.

Proposition 3.1. For any $\chi \in C_{c}^{\infty}\left(\Omega_{\text {int }}\right)$ such that $\chi(x)=1$ for $x$ in a neighborhood of $\left\{x_{1}, \ldots, x_{N}\right\}$, the unique solution $u$ of $(1.2)$ in $H^{1}\left(\mathbb{R}^{3}\right)$ satisfies

$$
u(x)=\chi(x) u_{0}(x)+\mathbb{E}_{x}\left[\int_{0}^{+\infty} g\left(X_{t}\right) \exp \left(-\int_{0}^{t} \kappa^{2}\left(X_{s}\right) \mathrm{d} s\right) \mathrm{d} t\right]
$$

for all $x \in \mathbb{R}^{3} \backslash\left\{x_{1}, \ldots, x_{N}\right\}$, where $\mathbb{P}_{x}$ is the law of the solution $\left(X_{t}, t \geq 0\right)$ of (2.17) such that $X_{0}=x$, and

$$
g(x)=\varepsilon_{\text {int }} u_{0}(x) \Delta \chi(x)+\varepsilon_{\text {int }} \nabla u_{0}(x) \cdot \nabla \chi(x) .
$$

A Monte Carlo method based on the formula (3.3) requires to discretize the process $X$ and to compute a discretization of the integral in (3.3). The process $X$ has (scaled) Brownian paths in $\Omega_{\text {int }}$ an $\Omega_{\text {ext }}$. The only discretization difficulty occurs when a path hits $\Gamma$. Very efficient simulation methods of Brownian paths are known, among which walk on spheres algorithms. These methods are efficient to simulate the time and position of exit from a domain. Here we need more, since the function $g$ is non-zero in $\Omega_{\text {int }}$.

Therefore, it is desirable to have a probabilistic interpretation that requires only a local information on the paths near $\Gamma$. The next probabilistic interpretation that we propose enjoys this property. 
Theorem 3.2. Assume that $\Gamma$ is a $C^{\infty}$ compact submanifold of $\mathbb{R}^{d}$. Fix $h>0$ and define $\Omega_{\mathrm{int}}^{h}=\left\{x \in \Omega_{\mathrm{int}}\right.$ : $-\rho(x)=\mathrm{d}(x, \Gamma) \geq h\}$, where $\mathrm{d}(x, \Gamma)$ denotes the distance between $x$ and the set $\Gamma$. For all $x \in \mathbb{R}^{3}$, let $\mathbb{P}_{x}$ denote the law of the solution $\left(X_{t}, t \geq 0\right)$ of (2.17) such that $X_{0}=x$, and define inductively the sequences of successive visit times of $\Omega_{\mathrm{int}}^{h}$ and $\Omega_{\mathrm{ext}}$

$$
\begin{aligned}
& \tau_{k}=\inf \left\{t \geq \tau_{k-1}^{\prime}: X_{t} \in \Omega_{\mathrm{int}}^{h}\right\} \\
\text { and } \quad \tau_{k}^{\prime} & =\inf \left\{t \geq \tau_{k}: X_{t} \in \Gamma\right\}
\end{aligned}
$$

for $k \geq 1$, where $\tau_{0}^{\prime}=0$. If $h$ is small enough to have $x_{j} \in \Omega_{\text {int }}^{h}$ for all $j \in\{1, \ldots, N\}$, then for all $x \in$ $\mathbb{R}^{3} \backslash\left\{x_{1}, \ldots, x_{N}\right\}$,

$$
u(x)=\mathbb{E}_{x}\left[\sum_{k=1}^{+\infty}\left(u_{0}\left(X_{\tau_{k}}\right)-u_{0}\left(X_{\tau_{k}^{\prime}}\right)\right) \exp \left(-\int_{0}^{\tau_{k}} \kappa^{2}\left(X_{t}\right) \mathrm{d} t\right)\right] .
$$

Before proving this result, let us comment it. First, the formula (3.4) is exact for all (sufficiently small) $h$. Therefore, once a discretization method has been chosen for the process $X$, one can choose the most convenient value of $h$ to estimate the r.h.s. of (3.4).

Second, due to the definition of $\kappa$ in (1.3), the r.h.s. of (3.4) only depends on the successive positions of $X_{\tau_{k}}$ and $X_{\tau_{k}^{\prime}}$ and on the time spent in $\Omega_{\text {ext }}$ by the process $X$.

Third, the successive positions of $X_{\tau_{k}}$ and $X_{\tau_{k}^{\prime}}$ belong to the small strip $\left\{x \in \mathbb{R}^{3}:-h \leq \rho(x) \leq 0\right\}$. This means that a careful discretization of the process is only needed in this small strip.

Fourth, the formula (3.4) is a non-standard probabilistic interpretation of the solution of an elliptic PDE, which can be easily generalized to other PDEs, and may provide new efficient numerical resolution methods.

Finally, all the algorithms proposed in Section 3.2 are well defined as soon as the normal at the boundary of $\Gamma$ is defined. The numerical study in Section 5.2 (partly) confirms the convergence of the methods, even if the assumption that $\Gamma$ is of class $C^{\infty}$ fails.

Proof. Since $\Delta\left(u-u_{0}\right)=0$ in $\Omega_{\text {int }}$, it follows from Theorem 2.18 (or, more simply, from the martingale property of harmonic functions of the Brownian motion) that, for all $x \in \Omega_{\mathrm{int}}^{h} \backslash\left\{x_{1}, \ldots, x_{N}\right\}, \tau_{1}=0$ and

$$
u(x)=\mathbb{E}_{x}\left[u\left(X_{\tau_{1}^{\prime}}\right)-u_{0}\left(X_{\tau_{1}^{\prime}}\right)\right]+u_{0}(x)
$$

Similarly, it follows from Theorem 2.18 that, for all $x \in \mathbb{R}^{3} \backslash \Omega_{\text {int }}^{h}$,

$$
u(x)=\mathbb{E}_{x}\left[u\left(X_{\tau_{1}}\right) \exp \left(-\int_{0}^{\tau_{1}} \kappa^{2}\left(X_{t}\right) \mathrm{d} t\right)\right] .
$$

Note that, if $\tau_{1}=+\infty$, then the exponential in the previous expectation is zero. The previous equality holds in particular if $x \in \Gamma$. Thus, applying the strong Markov property of $X$ (Thm. 2.14) and combining (3.5) and (3.6), we get

$$
u(x)=u_{0}(x)-\mathbb{E}_{x}\left[u_{0}\left(X_{\tau_{1}^{\prime}}\right)\right]+\mathbb{E}_{x}\left[u\left(X_{\tau_{2}}\right) \exp \left(-\int_{0}^{\tau_{2}} \kappa^{2}\left(X_{t}\right) \mathrm{d} t\right)\right], \quad \forall x \in \Omega_{\mathrm{int}}^{h} \backslash\left\{x_{1}, \ldots, x_{N}\right\} .
$$

Note that, in the previous formula, the time integral between 0 and $\tau_{2}$ is equal to the integral between $\tau_{1}^{\prime}$ and $\tau_{2}$ since $\kappa(x)=0$ for all $x \in \Omega_{\text {int }}$. 
Applying first (3.6) if $x \in \mathbb{R}^{3} \backslash \Omega_{\mathrm{int}}^{h}$ and next (3.7) recursively, we deduce from the strong Markov property that, for all $x \in \mathbb{R}^{3} \backslash\left\{x_{1}, \ldots, x_{N}\right\}$,

$$
\begin{aligned}
u(x) & =\mathbb{E}_{x}\left[\left(u_{0}\left(X_{\tau_{1}}\right)-u_{0}\left(X_{\tau_{1}^{\prime}}\right)\right) \mathrm{e}^{-\int_{0}^{\tau_{1}} \kappa^{2}\left(X_{t}\right) \mathrm{d} t}+u\left(X_{\tau_{2}}\right) \mathrm{e}^{-\int_{0}^{\tau_{2}} \kappa^{2}\left(X_{t}\right) \mathrm{d} t}\right] \\
& =\mathbb{E}_{x}\left[\sum_{i=1}^{m}\left(u_{0}\left(X_{\tau_{i}}\right)-u_{0}\left(X_{\tau_{i}^{\prime}}\right)\right) \mathrm{e}^{-\int_{0}^{\tau_{i}} \kappa^{2}\left(X_{t}\right) \mathrm{d} t}\right]+\mathbb{E}_{x}\left[u\left(X_{\tau_{m+1}}\right) \mathrm{e}^{-\int_{0}^{\tau_{m+1}} \kappa^{2}\left(X_{t}\right) \mathrm{d} t}\right] .
\end{aligned}
$$

By Proposition 2.15, $\left|X_{t}\right| \rightarrow+\infty$ a.s. as $t \rightarrow+\infty$. Since the process $X$ is continuous, this implies that, a.s., $\tau_{m}=+\infty$ for $m$ large enough. Since $u$ is bounded (Thm. 2.17) and $\kappa(x)=\bar{\kappa}>0$ for all $x \in \Omega_{\text {ext }}$, the last term of the r.h.s. of (3.8) converges to 0 by Lebesgue's theorem. Letting $m \rightarrow+\infty$ thus yields (3.4). Note also that the number of non-zero terms in the sum in the r.h.s. of (3.4) is a.s. finite.

\subsection{The Monte Carlo method proposed by Mascagni and Simonov and its extensions}

The Monte Carlo approximation of $u(x)$ and the associated discretization scheme of $X_{t}$ that we describe here have been originally proposed by Mascagni and Simonov in [23] to approximate the solution of PoissonBoltzmann's equation [34]. In this algorithm, after having hit $\Gamma$, the process jumps randomly and symmetrically with respect to $\Gamma$ (see below). Our goal is to propose various extensions of this algorithm with asymmetric jumps, and to analyse their performances (see Sect. 3.3). Note that other Monte Carlo approximations for this problem have been considered, among which the so-called "jump on spheres" method introduced by Simonov [33]. Since this algorithm is also based on a (nearly) symmetric jump approximation, for the sake of simplicity we choose to concentrate on the original Mascagni and Simonov's algorithm and its non-symmetric extensions.

Note that, in [23], the authors have not related their method to the solution of the SDE (2.17) and the probabilistic interpretation (3.4) of the PDE. Hence, our results justify their algorithm and allows us to propose some improvements.

The process $X$ has (scaled) Brownian paths in $\Omega_{\text {int }}$ and $\Omega_{\text {ext }}$, until it reaches $\Gamma$. We first describe the Walk on Spheres algorithm for the simulation of the path of $X$ inside $\Omega_{\text {ext }}$ and its variant, the Uncentered Walk on Spheres algorithm, used when $X$ is in $\Omega_{\text {int }}$.

Next, we detail the simulation procedure at the boundary $\Gamma$. The original method is detailed in Section 3.2.3.

\subsubsection{The Walk on Spheres (WOS) algorithm}

The Walk on Spheres (WOS) algorithm [31] provides an efficient simulation method of Brownian paths in a domain $D$. Given a position $y_{0} \in D$ of a Brownian path, the next position is obtained as follows:

- construct the largest open sphere $S_{0}=S\left(y_{0}, r_{0}\right)$ in $D$ with center $y_{0}$;

- the first point $y_{1}$ of exit from $S_{0}$ of a Brownian motion started from $y_{0}$ has the uniform law on $\partial S_{0}$;

- if needed, the law of the first exit time can also be obtained as a random variable $t_{0}$ independent of $y_{1}$ whose law has Laplace transform (cf. [4])

$$
\mathbb{E}\left(\exp \left(-\lambda t_{0}\right)\right)=\frac{r_{0} \sqrt{2 \lambda}}{\sinh \left(r_{0} \sqrt{2 \lambda}\right)} \quad \forall \lambda \geq 0
$$

Applying this inductively, we can construct a sequence of positions of a Brownian path in $\bar{D}$ and times between each positions $\left(y_{n}, t_{n}\right)_{n \geq 0}$ (see Fig. 3). Note also that the sequence of positions $\left(y_{n}\right)_{n \geq 0}$ of the Brownian path can be exactly simulated (which means that the only source of error in the simulation comes from the imperfection of the random number generator).

Note that, by the Feynman-Kac formula, for all $C^{2}$ function $v$ such that $\frac{1}{2} \Delta v-\lambda v=0$ in $D$,

$$
v\left(y_{0}\right)=\mathbb{E}\left(v\left(y_{1}\right) \exp \left(-\lambda t_{0}\right)\right)=\mathbb{E}\left(v\left(y_{1}\right)\right) \frac{r_{0} \sqrt{2 \lambda}}{\sinh \left(r_{0} \sqrt{2 \lambda}\right)}
$$




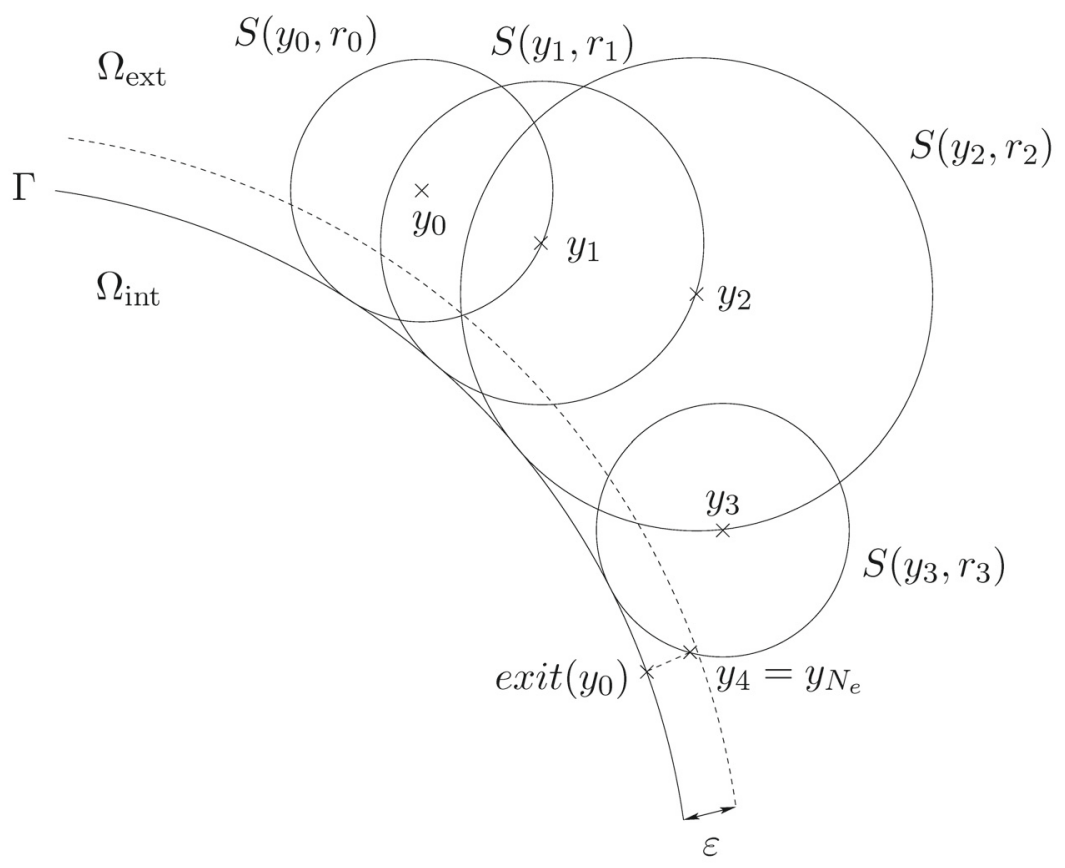

FiguRE 3. The WOS algorithm.

In this formula, the quantity $r_{0} \sqrt{2 \lambda} / \sinh \left(r_{0} \sqrt{2 \lambda}\right)$ may be interpreted as a probability of survival of the Brownian particle at the first step of the WOS algorithm. In other words, the parameter $\lambda$ may be interpreted as a rate of killing the Brownian particle.

Except in very special situations, the WOS algorithm a.s. never hits $\partial D$ after a finite number of steps. However, since in dimension 3 the norm of the Brownian motion a.s. goes to $+\infty$ when time goes to infinity, the sequence $\left(y_{n}\right)_{n \geq 0}$ either converges to some point in $\partial D$ or its norm converges to $+\infty$ (if $D$ is unbounded). In the first case, the common way to end the algorithm consists in introducing a small parameter $\varepsilon>0$ and stopping the WOS at the first step $n$ such that $\mathrm{d}\left(y_{n}, \partial D\right) \leq \varepsilon$. One then approximates the exit position as the closest point of $\partial D$ from $y_{n}$. In the case when $\left|y_{n}\right| \rightarrow+\infty$, the algorithm a.s. stops after a finite number of steps if the killing parameter $\lambda$ is positive. This leads to the following algorithm.

\section{WOS algorithm in the domain $D$.}

Set $k=0$. Given $y_{0} \in D, \lambda \geq 0$, and $\varepsilon>0$

(1) Let $S\left(y_{k}, r_{k}\right)$ be the largest open sphere included in $D$ centered at $y_{k}$.

(2) Sample $y_{k+1}$ according to the uniform distribution on $\partial S_{k}$.

(3) Kill the particle with probability $1-r_{k} \sqrt{2 \lambda} / \sinh \left(r_{k} \sqrt{2 \lambda}\right)$, and goto END if killed.

(4) IF $d\left(y_{k+1}, \partial D\right) \leq \varepsilon$, THEN set exit $\left(y_{0}\right)$ as the closest point of $\partial D$ from $y_{k+1}$ and goto END. ELSE, set $k=k+1$ and return to Step (1). END.

Denoting by $S_{k}$ the event for which the particle survives at the $k$ th step of the previous algorithm and applying the strong Markov property inductively, we have

$$
u\left(y_{0}\right)=\mathbb{E}\left(u\left(y_{1}\right) \mathbb{1}_{S_{1}}\right)=\ldots=\mathbb{E}\left(u\left(y_{k}\right) \mathbb{1}_{S_{k}}\right)
$$


for all $k \geq 0$. Therefore, denoting by $N_{e}$ the (random) number of steps in the algorithm,

$$
u\left(y_{0}\right)=\mathbb{E}\left(u\left(y_{N_{e}}\right) \mathbb{1}_{S_{N_{e}}}\right)=\mathbb{E}\left(u\left(\operatorname{exit}\left(y_{0}\right)\right) \mathbb{1}_{S_{N_{e}}}\right)+O(\varepsilon)
$$

under the assumption that the gradient of $u$ is uniformly bounded on $\bar{D}$. Note that the expected number of steps in the WOS algorithm generally grows as $|\log \varepsilon|$ [31]. Therefore, in practice, one can choose $\varepsilon$ of a smaller order of magnitude as the desired global accuracy without increasing much the computational cost.

\subsubsection{The Uncentered Walk on Spheres (UWOS) algorithm}

In the case where the domain $D$ is a finite union of spheres, the WOS can be modified in such a way that a path a.s. hits $\partial D$ after a finite number of steps, which provides an exact simulation technique of the first exit point. This algorithm is based on the observation that the density w.r.t. the uniform measure on $\partial S(c, R)$ of the first exit point of a Brownian motion from $S(c, R)$ started from $x \in S(c, R)$, is the Poisson kernel

$$
\frac{R^{2}-|y-c|^{2}}{4 \pi R|y-x|^{3}}
$$

Expressed in the spherical coordinates $(r, \theta, \varphi)$ centered at $c$ and such that $x$ has coordinates $(r, 0,0)$ where $r=|x-c|$, the exit point has coordinates $(R, \theta, \varphi)$, where $\theta$ has the uniform law on $[0,2 \pi]$ and $\varphi$ is independent of $\theta$ with cumulative distribution function

$$
F_{R, r}(\alpha):=\mathbb{P}(\varphi \leq \alpha)=\frac{R^{2}-r^{2}}{2 R r}\left(\frac{R}{R-r}-\frac{R}{\sqrt{R^{2}-2 R r \cos \alpha+r^{2}}}\right)
$$

which is explicitly invertible.

Then, the following modification of the WOS algorithm allows one to simulate exactly the exit point of $D=S\left(c_{1}, r_{1}\right) \cup \ldots \cup S\left(c_{n}, r_{n}\right)$ after an a.s. finite number of steps.

\section{Uncentered WOS (UWOS) algorithm.}

Set $k=0$. Given $y_{0} \in D$,

(1) Choose $i \in\{1, \ldots, n\}$ such that $y_{k} \in S\left(c_{i}, r_{i}\right)$.

(2) Simulate $y_{k+1}=\left(r_{i}, \theta, \varphi\right)$ where $\theta$ is uniform on $[0,2 \pi]$ and $\varphi$ is independent of $\theta$ with cumulative distribution function $F_{r_{i},\left|y_{k}-c_{i}\right|}$, in the spherical coordinates centered at $c_{i}$ such that $y_{k}=\left(\left|y_{k}-c_{i}\right|, 0,0\right)$.

(3) IF $y_{k+1} \in \partial D$, THEN set exit $\left(y_{0}\right)=y_{k+1}$ and goto END.

ELSE, set $k=k+1$ and return to Step (1).

END.

If needed, the Laplace transform of the law of the exit time can also be computed, but it is not independent of the exit position, which leads to complications. However, in our case, the previous version of the UWOS algorithm will be sufficient.

\subsubsection{Discretization of $X_{t}$ at the boundary}

The UWOS algorithm can be used to simulate exactly the paths of $X$ in $\Omega_{\text {int }}$ until it hits $\Gamma$, and the WOS algorithm to simulate the paths of $X$ in $\Omega_{\text {ext }}$ until it reaches $\Gamma$, with a constant rate of killing.

The last ingredient of the algorithm is the discretization procedure to move from a point on $\Gamma$ to a point in $\Omega_{\text {int }} \cup \Omega_{\text {ext }}$.

The method proposed by Mascagni and Simonov [23] is the following: let $x \in \Gamma$ and $h>0$. Recall that $n(x)$ denotes the unit vector normal to $\Gamma$ at $x$ pointing towards $\Omega_{\text {ext }}$. The new position of the discretized process 
started from $x \in \Gamma$ is

$$
p(x)= \begin{cases}x+h n(x) & \text { with probability } \frac{\varepsilon_{\mathrm{ext}}}{\varepsilon_{\mathrm{int}}+\varepsilon_{\mathrm{ext}}} \\ x-h n(x) & \text { with probability } \frac{\varepsilon_{\mathrm{int}}}{\varepsilon_{\mathrm{int}}+\varepsilon_{\mathrm{ext}}}\end{cases}
$$

This construction is deduced from the following observation: let $u$ be the solution of (1.2); Taylor expansions and the transmission condition (2.15) lead to

$$
u(x)=\frac{\varepsilon_{\mathrm{int}}}{\varepsilon_{\mathrm{int}}+\varepsilon_{\mathrm{ext}}} u(x-h n(x))+\frac{\varepsilon_{\mathrm{ext}}}{\varepsilon_{\mathrm{int}}+\varepsilon_{\mathrm{ext}}} u(x+h n(x))+O\left(h^{2}\right)=\mathbb{E}[u(p(x))]+O\left(h^{2}\right)
$$

when the second-order exterior and interior normal derivatives of $u_{i}$ and $u_{e}$ on $\Gamma$ are bounded (which holds true if $\Gamma$ is $C^{\infty}$, as proved in Thm. 2.17).

In view of the probabilistic interpretation of $u$ in Theorem 3.2, this leads to the algorithm below. Because of the definition of $p(x)$ in (3.11), we call it the Symmetric Normal Jump algorithm.

\section{Symmetric Normal Jump (SNJ) algorithm.}

Given $x_{0} \in \Omega_{\text {int }}$,

Set $k=0$ and score $=0$.

(1) IF $x_{k} \in \Omega_{\text {int }}$,

(a) THEN use the UWOS algorithm to simulate exit $\left(x_{k}\right)$ and set score $=$ score $-u_{0}\left(\operatorname{exit}\left(x_{k}\right)\right)$,

(b) ELSE use the WOS algorithm with $\lambda=\bar{\kappa}^{2} / 2 \varepsilon_{\text {ext }}$ to simulate $\operatorname{exit}\left(x_{k}\right)$.

IF the particle has been killed, THEN goto END.

(2) Let $x_{k+1}=p\left(\operatorname{exit}\left(x_{k}\right)\right)$ as in (3.11).

(3) IF $x_{k+1} \in \Omega_{\text {int }}$, THEN set score $=$ score $+u_{0}\left(x_{k+1}\right)$.

(4) Set $k=k+1$ and return to Step (1).

END.

Let $\left\{\right.$ score $_{1}, \ldots$, score $\left._{M}\right\}$ be the scores obtained by $M$ independent runs of the SNJ algorithm. The Monte Carlo estimation of $u\left(x_{0}\right)-u_{0}\left(x_{0}\right)$ is then obtained as

$$
u\left(x_{0}\right)-u_{0}\left(x_{0}\right) \approx \frac{1}{M} \sum_{j=1}^{M} \text { score }_{j}
$$

Note that this algorithm can be extended to a general $\Omega_{\text {int }}$ (not necessarily a union of spheres) by replacing the UWOS algorithm in Step (2a) by the WOS algorithm in $\Omega_{\text {int }}$.

We denote by $\bar{u}_{h}^{\mathrm{SNJ}}\left(x_{0}\right)$ the expectation of the score obtained by the SNJ algorithm when the initial position of the particle is $x_{0}$.

\subsection{Extensions of the SNJ algorithm}

The WOS and UWOS algorithms described in Section 3.2 are exact (up to the error due to the parameter $\varepsilon$ ). However, the discretization of $X_{t}$ on $\Gamma$ is not. We propose the following improvements of this step of the algorithm.

\subsubsection{Asymmetric Normal Jump (ANJ) algorithm}

Our first extension is an asymmetric version of the SNJ algorithm. The asymmetry parameter $\alpha>0$ is chosen by the user. 
$\operatorname{ANJ}(\alpha)$ algorithm.

This algorithm is the SNJ algorithm where the random variable $p(x)$ in Step (2) is modified as

$$
p(x)= \begin{cases}x+\alpha h n(x) & \text { with probability } \frac{\varepsilon_{\text {ext }}}{\varepsilon_{\text {ext }}+\alpha \varepsilon_{\text {int }}} \\ x-h n(x) & \text { with probability } \frac{\alpha \varepsilon_{\text {int }}}{\varepsilon_{\text {ext }}+\alpha \varepsilon_{\text {int }}}\end{cases}
$$

for all $x \in \Gamma$.

The choice of the probability $\varepsilon_{\text {ext }} /\left(\varepsilon_{\text {ext }}+\alpha \varepsilon_{\text {int }}\right)$ comes from the expansion

$$
\left(\varepsilon_{\mathrm{ext}}+\alpha \varepsilon_{\mathrm{int}}\right) u(x)=\varepsilon_{\mathrm{ext}} u(x+\alpha h n(x))+\alpha \varepsilon_{\mathrm{int}} u(x-h n(x))+O\left(h^{2}\right),
$$

valid when the second-order interior and exterior normal derivatives of $u$ on $\Gamma$ are bounded.

As will appear in the error and performance analysis of Section 4 and in the numerical results of Section 5 , the interest of this method is that, when $\alpha>1$, the particle is moved further away from $\Gamma$ than in the SNJ algorithm when it jumps in $\Omega_{\text {ext }}$, which increases the probability that the particle is killed. Therefore, this method reduces the computational cost, but has a larger bias. The trade-off is numerically analyzed in Section 5 .

We denote by $\bar{u}_{h}^{\mathrm{ANJ}(\alpha)}\left(x_{0}\right)$ the expectation of the score obtained by the ANJ algorithm with parameter $\alpha>0$ when the initial position of the particle is $x_{0}$.

\subsubsection{Unbiased Asymmetric Normal Jump (UANJ) algorithm}

The following improved algorithm is due to the geometric specificity of the problem: $\Omega_{\text {int }}$ is an union of spheres.

In the case where $\Omega_{\text {int }}$ is a single sphere in Section 4.1, this improvement removes all the bias of the algorithm (except for the bias coming from the parameter $\varepsilon$ ), as proved in Section 4.1.1. For this reason, we call this algorithm the Unbiased ANJ (or UANJ) algorithm.

Assume that $\Omega_{\text {int }}=S(0, R)$, for $R>0$, and fix $x \in \Gamma$. Define

$$
\tau_{h, \alpha}:=\inf \left\{t \geq 0:\left|X_{t}\right|=R-h \text { or }\left|X_{t}\right|=R+\alpha h\right\}
$$

and let

$$
\tau_{h, \alpha}^{\text {ext }}:=\int_{0}^{\tau_{h, \alpha}} \mathbb{1}_{\left\{X_{t} \in \Omega_{\text {ext }}\right\}} \mathrm{d} t
$$

be the amount of time spent by the process $X$ in $\Omega_{\text {ext }}$ on the time interval $\left[0, \tau_{h, \alpha}\right]$. The joint distribution of $\left|X_{\tau_{h, \alpha}}\right|$ and $\exp \left(-\bar{\kappa}^{2} \tau_{h, \alpha}^{\text {ext }}\right)$ is independent of the initial position $x$ on $\partial B(0, R)$ and is explicitly known (see below). This leads to the following algorithm.

\section{$\operatorname{UANJ}(\alpha)$ algorithm.}

This algorithm is the SNJ algorithm where Step (2) is replaced by

(2) (a) With probability $\mathbb{P}_{x}\left(\left|X_{\tau_{h, \alpha}}\right|=R-h\right)$ defined below in (3.12), let $x_{k+1}=p\left(\operatorname{exit}\left(x_{k}\right)\right)=\operatorname{exit}\left(x_{k}\right)-h n\left(\operatorname{exit}\left(x_{k}\right)\right)$.

Then, kill the particle with probability $1-\mathbb{E}_{x}\left(\exp \left(-\bar{\kappa}^{2} \tau_{h, \alpha}^{\text {ext }}\right)|| X_{\tau_{h, \alpha}} \mid=R-h\right)$ defined in (3.13).

(b) Otherwise, let $x_{k+1}=p\left(\operatorname{exit}\left(x_{k}\right)\right)=\operatorname{exit}\left(x_{k}\right)+\alpha h n\left(\operatorname{exit}\left(x_{k}\right)\right)$. Then, kill the particle with probability $1-\mathbb{E}_{x}\left(\exp \left(-\bar{\kappa}^{2} \tau_{h, \alpha}^{\text {ext }}\right)|| X_{\tau_{h, \alpha}} \mid=R+\alpha h\right)$ defined in (3.14).

(c) IF the particle is killed, THEN goto END.

Note that this algorithm can be extended to the case of an arbitrary number of spheres $S\left(c_{i}, r_{i}\right), \ldots, S\left(c_{n}, r_{n}\right)$ by applying the same rule when $x_{k} \in \partial S\left(c_{i}, r_{i}\right)$, but replacing $R$ by $r_{i}$. 
We denote by $\bar{u}_{h}^{\mathrm{UANJ}(\alpha)}\left(x_{0}\right)$ the expectation of the score given by this algorithm when the initial position of the particle is $x_{0}$.

Even if we prove below that the only bias in the previous algorithm comes from the parameter $\varepsilon$ in the case of a single sphere, this does not necessarily holds true in the case where $\Omega_{\text {int }}$ is the union of several spheres.

The joint distribution of the random variables $\left|X_{\tau_{h, \alpha}}\right|$ and $\exp \left(-\bar{\kappa}^{2} \tau_{h, \alpha}^{\text {ext }}\right)$ are explicited as follows. First, we deduce from Itô-Meyer's formula that $Y_{t}=\left|X_{t}\right|-R$ solves the one-dimensional SDE

$$
\mathrm{d} Y_{t}=\sqrt{2 \varepsilon^{\#}\left(Y_{t}\right)} \mathrm{d} W_{t}+\frac{2 \varepsilon^{\#}\left(Y_{t}\right)}{Y_{t}+R} \mathrm{~d} t+\frac{\varepsilon_{\text {ext }}-\varepsilon_{\text {int }}}{2 \varepsilon_{\text {ext }}} \mathrm{d} L_{t}^{0}(Y)
$$

where $\varepsilon^{\#}$ is defined in $(2.6)$ and

$$
W_{t}=\int_{0}^{t} \frac{X_{s}}{\left|X_{s}\right|} \mathrm{d} B_{s}
$$

is a standard Brownian motion. Therefore, thanks to Theorem 2.18 with $d=1, v(y)=\mathbb{P}_{y}\left(Y_{\tau_{h, \alpha}}=R-h\right)$ satisfies

$$
\left(\varepsilon^{\#} v^{\prime}\right)^{\prime}(y)+\frac{2 \varepsilon^{\#}(y)}{y+R} v^{\prime}(y)=0
$$

with $v(-h)=1$ and $v(\alpha h)=0$. It then follows from elementary computations that, for all $x \in \Gamma$,

$$
\mathbb{P}_{x}\left(\left|X_{\tau_{h, \alpha}}\right|=R-h\right)=\mathbb{P}\left(Y_{\tau_{h, \alpha}}=R-h \mid Y_{0}=0\right)=\frac{\alpha \varepsilon_{\text {int }}\left(1-\frac{h}{R}\right)}{\alpha \varepsilon_{\text {int }}+\varepsilon_{\text {ext }}+\left(\varepsilon_{\text {ext }}-\varepsilon_{\text {int }}\right) \frac{\alpha h}{R}} .
$$

Note that this probability depends on $h$, but its first-order expansion when $h \rightarrow 0$ agrees with the corresponding probability of the $\operatorname{ANJ}(\alpha)$ algorithm.

Using Feynman-Kac's formula again, $v(y)=\mathbb{E}\left(\exp \left(-\bar{\kappa}^{2} \tau_{h, \alpha}^{\text {ext }}\right) \mathbb{1}_{\left\{Y_{\tau_{h, \alpha}}=-h\right\}} \mid Y_{0}=y\right)$ solves

$$
\left(\varepsilon^{\#} v^{\prime}\right)^{\prime}(y)+\frac{2 \varepsilon^{\#}(y)}{y+R} v^{\prime}(y)=\kappa^{\#}(y)^{2} v(y)
$$

with boundary conditions $v(-h)=1$ and $v(\alpha h)=0$, where $\kappa^{\#}(y)=0$ if $y<0$ and $\kappa^{\#}(y)=\bar{\kappa}$ if $y \geq 0$. The solution of this differential equation is

$$
v(y)= \begin{cases}\frac{a y+b}{y+R} & \text { if } y \leq 0 \\ \frac{c \cosh \left(\mu_{\mathrm{ext}} y\right)+d \sinh \left(\mu_{\mathrm{ext}} y\right)}{y+R} & \text { if } y>0\end{cases}
$$

where $\mu_{\text {ext }}=\bar{\kappa} / \sqrt{\varepsilon_{\text {ext }}}$ and the constants $a, b, c, d$ satisfy

$$
\begin{aligned}
b & =c, & \varepsilon_{\mathrm{int}}(a R-b) & =\varepsilon_{\mathrm{ext}}\left(d \mu_{\mathrm{ext}} R-c\right), \\
b-a h & =R-h, & c \cosh \left(\mu_{\mathrm{ext}} \alpha h\right) & =-d \sinh \left(\mu_{\mathrm{ext}} \alpha h\right) .
\end{aligned}
$$

This system of equations can be solved explicitly: for all $x \in \Gamma$,

$$
\begin{aligned}
\mathbb{E}_{x}\left(\mathrm{e}^{-\bar{\kappa}^{2} \tau_{h, \alpha}^{\mathrm{ext}}}|| X_{\tau_{h, \alpha}} \mid=R-h\right) & =\frac{b}{R \mathbb{P}\left(Y_{\tau_{h, \alpha}}=R-h \mid Y_{0}=0\right)} \\
& =\frac{\alpha \varepsilon_{\mathrm{int}}+\varepsilon_{\mathrm{ext}}+\left(\varepsilon_{\mathrm{ext}}-\varepsilon_{\mathrm{int}}\right) \frac{\alpha h}{R}}{\alpha \varepsilon_{\mathrm{int}}+\varepsilon_{\mathrm{ext}} \frac{\mu_{\mathrm{ext}} \alpha h}{\tanh \left(\mu_{\mathrm{ext}} \alpha h\right)}+\left(\varepsilon_{\mathrm{ext}}-\varepsilon_{\mathrm{int}}\right) \frac{\alpha h}{R}} .
\end{aligned}
$$


Similarly, for all $x \in \Gamma$,

$$
\mathbb{E}_{x}\left(\mathrm{e}^{-\bar{\kappa}^{2} \tau_{h, \alpha}^{\text {ext }}}|| X_{\tau_{h, \alpha}} \mid=R+\alpha h\right)=\frac{\varepsilon_{\text {ext }}+\alpha \varepsilon_{\text {int }}+\left(\varepsilon_{\text {ext }}-\varepsilon_{\text {int }}\right) \frac{\alpha h}{R}}{\varepsilon_{\text {ext }} \cosh \left(\mu_{\text {ext }} \alpha h\right)+\varepsilon_{\text {int }} \frac{\sinh \left(\mu_{\text {ext }} \alpha h\right)}{\mu_{\text {ext }} h}+\left(\varepsilon_{\text {ext }}-\varepsilon_{\text {int }}\right) \frac{\sinh \left(\mu_{\text {ext }} \alpha h\right)}{\mu_{\text {ext }} R}} .
$$

\subsubsection{Neutron Transport Jump (NTJ) algorithm}

All the previous algorithms have the property that, at times where the particle reaches $\Gamma$, one samples the next position in the normal direction to $\Gamma$. However, it is natural to take into account possible lateral displacements when the particle jumps from $\Gamma$. This is typically the case in neutron transport approximations of diffusion processes, which naturally suggest a new way to move the particle from $\Gamma$. This idea has been introduced in [16]. Define

$$
T_{h} u_{h}(x, v)=-\frac{v}{h} \nabla_{x} u_{h}(x, v)+\kappa^{2} u_{h}(x, v)+\frac{1}{3 h^{2} \varepsilon(x)}\left(\frac{1}{4 \pi} \int_{S_{2}} u_{h}\left(x, v^{\prime}\right) \mathrm{d} v^{\prime}-u_{h}(x, v)\right),
$$

the neutron transport operator with absorption boundary conditions (no incoming neutrons) in $D \times S^{2}$ where $D$ is a bounded open domain of $\mathbb{R}^{3}$. When $\varepsilon(x)$ is smooth enough, the solution $u_{h} \in C\left(\mathbb{R}^{6}\right)$ of the equation

$$
T_{h} u_{h}=f
$$

for $f$ smooth enough, converges in $L^{\infty}\left(D \times S^{2}\right)$ to the solution $u$ of the Poisson equation

$$
-\nabla \cdot(\varepsilon(x) \nabla u)+\kappa^{2} u=f
$$

in $D$ with Dirichlet boundary conditions (see [6], Chap. 21).

In our case, the function $\varepsilon(x)$ is discontinuous, but one can still define the approximation, even though so far this procedure has no theoretical justification. The neutron transport approximation leads to two possible approximations procedures: a global one, which consists in replacing the diffusion process by a transport process in the domain $D$, and a local one, which uses the transport approximation only at the interface $\Gamma$, and an efficient Brownian path simulation elsewhere.

As suggested by (3.15), we construct the local approximation as follows. Assume that $X_{0}=x \in \Gamma$, fix a parameter $\hat{h}>0$ and sample a random vector $v$ uniformly on the unit sphere $S^{2}$ and an independent exponential random variable $E$ with parameter 1 . Then, the next position of the particle is

$$
p(x)= \begin{cases}x+3 \varepsilon_{\mathrm{int}} \hat{h} E v & \text { if } v \cdot n(x)<0\left(\text { with probability } \frac{1}{2}\right), \\ x+3 \varepsilon_{\mathrm{ext}} \hat{h} E v & \text { otherwise. }\end{cases}
$$

This corresponds to a velocity $v / \hat{h}$ and a collision time $T$ equal to $3 E \hat{h}^{2} \varepsilon_{\text {int }}$ inside $\Omega_{\text {int }}$ and $3 E \hat{h}^{2} \varepsilon_{\text {ext }}$ outside. Note that, for $n(x)$ to be well-defined, we need (and implicitly assume) $\Gamma$ to be a $C^{1}$ submanifold of $\mathbb{R}^{3}$. If $\Gamma$ is only piecewise $C^{1}$ (for example when $\Omega_{\text {int }}$ is the union of several spheres), then one needs to specify $p(x)$ at the points $x$ where $\Gamma$ is not $C^{1}$. However, in the numerical tests we did in Section 5 the simulated positions of the particle never hit this set of points (which has Lebesgue measure 0).

In order to be able to compare the NTJ algorithm with the previous ones, we set $h=3 \varepsilon_{\text {int }} \hat{h} / 2$. It can then be checked that

$$
\mathbb{E}\left(3 \hat{h} v_{x} E \varepsilon_{\text {int }} \mid v \cdot n(x)<0\right)=-h+o(h) \quad \text { and } \quad \mathbb{E}\left(3 \hat{h} v_{x} E \varepsilon_{\text {ext }} \mid v \cdot n(x)>0\right)=\alpha h+o(h)
$$

as in the previous algorithms, where $\alpha=\varepsilon_{\text {ext }} / \varepsilon_{\text {int }}$. 


\section{NTJ algorithm.}

This algorithm is the SNJ algorithm where Step (2) is replaced by

(2) (a) Draw $v_{k}$ uniformly on $S^{2}$ and $E_{k}$ according to the exponential distribution with parameter 1 .

(b) Set

$$
x_{k+1}=p\left(\operatorname{exit}\left(x_{k}\right)\right)= \begin{cases}\operatorname{exit}\left(x_{k}\right)+2 h E_{k} v_{k} & \text { if } v_{k} \cdot n\left(\operatorname{exit}\left(x_{k}\right)\right)<0 \\ \operatorname{exit}\left(x_{k}\right)+2 \alpha h E_{k} v_{k} & \text { otherwise }\end{cases}
$$

(c) Define the collision time

$$
T_{k}= \begin{cases}4 h^{2} E_{k} /\left(3 \varepsilon_{\text {int }}\right) & \text { if } v_{k} \cdot n\left(\operatorname{exit}\left(x_{k}\right)\right)<0, \\ 4 \alpha h^{2} E_{k} /\left(3 \varepsilon_{\text {int }}\right) & \text { otherwise }\end{cases}
$$

IF $v_{k} \cdot n\left(\operatorname{exit}\left(x_{k}\right)\right) \geq 0$, THEN kill the particle with probability

$$
1-\exp \left(-\int_{0}^{T_{k}} \bar{\kappa}^{2} \mathrm{~d} s\right)=1-\exp \left(-\frac{4 \bar{\kappa}^{2} \alpha h^{2} E_{k}}{3 \varepsilon_{\text {int }}}\right) .
$$

IF the particle is killed, THEN goto END.

Step (2c) is motivated by the fact that the neutron transport algorithm also approximates the time needed by the particle to jump from $\Gamma$ to its next position (in the neutron transport approximation, a jump actually corresponds to a constant velocity motion on a straight line). Since $\bar{\kappa}^{2}$ can be interpreted as a rate of killing in $\Omega_{\text {ext }}$ in the Feynman-Kac representation (3.4), this justifies the killing probability (3.18). As shown by the bias computations of Section 4.1 and the numerical tests of Section 5, adding this killing step improves substantially the performance of the NTJ algorithm.

Note finally that, when $\varepsilon_{\text {ext }}$ is larger than $\varepsilon_{\text {int }}$ (which is usually the case in the molecular dynamics context), the particle is moved further away from the molecule than in the SNJ algorithm, which increases the probability that the particle gets killed before coming back to $\Gamma$.

For more details about Monte Carlo simulations of neutron transport processes, we refer to $[13,19,20]$.

We denote by $\bar{u}_{h}^{\mathrm{NTJ}}\left(x_{0}\right)$ the expectation of the score obtained by the NTJ algorithm when the initial position of the particle is $x_{0}$.

\section{ERror ANALYSIS}

In this section, we analyse the convergence of the previous algorithms, first in the case of one sphere, and then in the general case.

\subsection{The one sphere case}

We assume that $\Omega_{\text {int }}=S(0, R)$ with $R>0, N=1, x_{1}=0$ and $q_{1}=q$. In this case, the solution of Poisson-Boltzmann's PDE (1.2) can be explicitly computed:

$$
u(x)= \begin{cases}\frac{1}{4 \pi}\left(\frac{1}{\varepsilon_{\mathrm{int}}|x|}-\frac{1}{\varepsilon_{\mathrm{int}} R}+\frac{1}{\varepsilon_{\mathrm{ext}} \beta R}\right) & \text { if } x \in \Omega_{\mathrm{int}} \backslash\{0\}, \\ \frac{\exp \left(-\frac{\bar{\kappa}}{\sqrt{\varepsilon_{\mathrm{ext}}}}(|x|-R)\right)}{4 \pi \varepsilon_{\mathrm{ext}} \beta|x|} & \text { if } x \in \Omega_{\mathrm{ext}} \cup \Gamma,\end{cases}
$$


where

$$
\beta=1+\frac{\bar{\kappa} R}{\sqrt{\varepsilon_{\mathrm{ext}}}}
$$

\subsubsection{Estimation of the bias}

Due to the spherical symmetry of the problem, the only relevant information in Steps (2) of all the algorithms of the previous section is the pair of random variables $(A, B)$, where $A=\rho(p(x))$ is the distance from $\Gamma$ of the next position of the particle, and $B \in\{0,1\}$ is a random variable such that the particle is killed iff $B=0$. Note that $A$ and $B$ may not be independent (for example in the $\operatorname{UANJ}(\alpha)$ and NTJ algorithms).

Note that, in all our algorithms, the law of $(A, B)$ is independent of $x \in \partial B(0, R), A \neq 0$ and $A>-R$ a.s. Given any pair of random variables $(A, B)$ satisfying these properties, we consider a sequence of i.i.d. r.v. $\left(A_{k}, B_{k}\right)_{k \geq 1}$ having the same law as $(A, B)$ and the following generic algorithm.

\section{Generic algorithm.}

This algorithm is the SNJ algorithm where Step (2) is replaced by

(2) Let $x_{k+1}=\operatorname{exit}\left(x_{k}\right)+A_{k} n\left(x_{k}\right)$ and the particle is killed if $B_{k}=0$.

We denote by $\bar{u}_{(A, B)}\left(x_{0}\right)$ the expectation of the score obtained by this algorithm starting from $x_{0} \in \mathbb{R}^{3}$.

Theorem 4.1. Assume that the pair of r.v. $(A, B)$ satisfy the previous assumptions. Then, for all $x_{0} \in \Omega_{\mathrm{int}}$,

$$
\bar{u}_{(A, B)}\left(x_{0}\right)-\left(u-u_{0}\right)\left(x_{0}\right)=\frac{\mathbb{E}[B u((R+A, 0,0))]-u((R, 0,0))+O(\varepsilon)}{\mathbb{P}(B=0)+\frac{\beta}{R} \mathbb{E}\left(B A \mathbb{1}_{A>0}\right)+O\left(\varepsilon+\mathbb{E}\left(A^{2}\right)\right)} .
$$

Proof. Let $x_{0} \in \Omega_{\text {int }}$ and $0<h<\mathrm{d}\left(x_{0}, \Gamma\right)$. On the one hand, Theorem 3.2 leads to

$$
u\left(x_{0}\right)-u_{0}\left(x_{0}\right)=\mathbb{E}_{x_{0}}\left[-u_{0}\left(X_{\tau_{1}^{\prime}}\right)+\sum_{j=2}^{+\infty} \mathrm{e}^{-\int_{0}^{\tau_{j}} \kappa^{2}\left(X_{t}\right) \mathrm{d} t}\left(u_{0}\left(X_{\tau_{j}}\right)-u_{0}\left(X_{\tau_{j}^{\prime}}\right)\right)\right] .
$$

On the other hand, denoting by $L_{k}$ the event "the particle is still alive in the generic algorithm when in state $x_{k}$ ", we have

$$
\bar{u}_{(A, B)}\left(x_{0}\right)=\mathbb{E}\left[-u_{0}\left(\operatorname{exit}\left(x_{0}\right)\right)+\sum_{k=1}^{+\infty} \mathbb{1}_{\left\{x_{k} \in \Omega_{\mathrm{int}}\right\}} \mathbb{1}_{L_{k}}\left(u_{0}\left(x_{k}\right)-u_{0}\left(\operatorname{exit}\left(x_{k}\right)\right)\right)\right] .
$$

Defining $k(j):=\inf \left\{k>k(j-1): x_{k} \in \Omega_{\text {int }}\right\}$ for all $j \geq 1$ with the convention that $k(0)=0$, the preceding equality may be written as

$$
\bar{u}_{(A, B)}\left(x_{0}\right)=\mathbb{E}\left[-u_{0}\left(\operatorname{exit}\left(x_{0}\right)\right)+\sum_{j=1}^{+\infty} \mathbb{1}_{L_{k(j)}}\left(u_{0}\left(x_{k(j)}\right)-u_{0}\left(\operatorname{exit}\left(x_{k(j)}\right)\right)\right)\right]
$$

which is similar to (4.4). Extend the definition of $\bar{u}_{(A, B)}\left(x_{0}\right)$ to all $x_{0} \in \mathbb{R}^{3}$ by

$$
\bar{u}_{(A, B)}\left(x_{0}\right)=\mathbb{E}\left[-\mathbb{1}_{\left\{x_{0} \in \Omega_{\mathrm{int}} \cup \Gamma\right\}} u_{0}\left(\operatorname{exit}\left(x_{0}\right)\right)+\sum_{k=1}^{+\infty} \mathbb{1}_{\left\{x_{k} \in \Omega_{\mathrm{int}}\right\}} \mathbb{1}_{L_{k}}\left(u_{0}\left(x_{k}\right)-u_{0}\left(\operatorname{exit}\left(x_{k}\right)\right)\right)\right],
$$

and set $w(|x|)=u(x), w_{0}(|x|)=u_{0}(x)$ and $\bar{w}(|x|)=\bar{u}_{(A, B)}(x)$ for all $x \in \mathbb{R}^{3}$ (all these functions are well-defined because of the spherical symmetry of the problem). Note that

$$
\left.\bar{w}\left(\left|x_{0}\right|\right)=\mathbb{E}\left[-\mathbb{1}_{\left\{x_{0} \in \Omega_{\mathrm{int}} \cup \Gamma\right\}} w_{0}(R)+\sum_{k=1}^{+\infty} \mathbb{1}_{\left\{x_{k} \in \Omega_{\mathrm{int}}\right\}} \mathbb{1}_{L_{k}}\left(w_{0}\left(\left|x_{k}\right|\right)-w_{0}(R)\right)\right)\right]
$$


and that $\bar{w}$ is constant on $[0, R]$. For all $x_{0} \in \Omega_{\text {int }}$, in view of (3.5), we thus have

$$
\bar{w}\left(\left|x_{0}\right|\right)-\left(w-w_{0}\right)\left(\left|x_{0}\right|\right)=\bar{w}(R)-w(R)+w_{0}(R) .
$$

Next,

$$
\begin{aligned}
\bar{w}(R)-w(R)+w_{0}(R)= & -w(R)+\mathbb{E}\left[\mathbb{1}_{\left\{x_{1} \in \Omega_{\mathrm{int}}\right\}} \mathbb{1}_{L_{1}} w_{0}\left(\left|x_{1}\right|\right)\right] \\
& +\mathbb{E}\left[\mathbb{1}_{L_{1}}\left(-\mathbb{1}_{\left\{x_{1} \in \Omega_{\mathrm{int}}\right\}} w_{0}(R)+\sum_{k=2}^{+\infty} \mathbb{1}_{\left\{x_{k} \in \Omega_{\mathrm{int}}\right\}} \mathbb{1}_{L_{k}}\left(w_{0}\left(\left|x_{k}\right|\right)-w_{0}(R)\right)\right)\right] \\
= & -w(R)+\mathbb{E}\left[B \mathbb{1}_{\{A<0\}} w_{0}(R+A)\right]+\mathbb{E}[B \bar{w}(R+A)] .
\end{aligned}
$$

Since, by definition of $\bar{w}$,

$$
\bar{w}(R+a)=\mathbb{P}\left(L_{1}|| x_{0} \mid=R+a\right)\left(\bar{w}(R)+w_{0}(R)\right) \quad \forall a>0,
$$

we obtain that

$$
\begin{aligned}
\bar{w}(R)-w(R)+w_{0}(R)= & \mathbb{E}\left[B \mathbb{1}_{\{A<0\}}\left(\bar{w}(R)-w(R)+w_{0}(R)\right)\right]+\mathbb{E}\left[B \mathbb{1}_{\{A<0\}} w(R+A)\right] \\
& +\mathbb{E}\left[B \mathbb{1}_{\{A>0\}} \mathbb{P}\left(L_{1} \mid x_{0}=R+A\right)\left(\bar{w}(R)-w(R)+w_{0}(R)\right)\right] \\
& +\mathbb{E}\left[B \mathbb{1}_{\{A>0\}} w(R) \mathbb{P}\left(L_{1}|| x_{0} \mid=R+A\right)\right]-w(R) .
\end{aligned}
$$

By (3.10), we have

$$
w(R+a)=w(R) \mathbb{P}\left(L_{1}|| x_{0} \mid=R+a\right)+O(\varepsilon) \quad \forall a>0
$$

since $w^{\prime}$ is bounded on $[R, \infty)$. Therefore,

$$
\begin{aligned}
\bar{u}_{(A, B)}\left(x_{0}\right)-\left(u-u_{0}\right)\left(x_{0}\right)=\bar{w}(R)-w(R)+ & w_{0}(R) \\
& =\frac{\mathbb{E}[B w(R+A)]-w(R)+O(\varepsilon)}{1-\mathbb{P}(A<0, B=1)-\mathbb{E}\left[B \mathbb{1}_{\{A>0\}} \mathbb{P}\left(L_{1} \mid x_{0}=R+A\right)\right]} .
\end{aligned}
$$

The proof of Theorem 4.1 will then be completed by computing the first-order expansion of the denominator in powers of $A$.

Letting $y_{0}=(R+a, 0,0) \in \mathbb{R}^{3}$ with $a>\varepsilon$, we may define the events $S_{i}$, the positions $y_{i}$ and the radii $r_{i}$ as in the WOS algorithm of Section 3.2.1. Let us also denote by $t_{i}$ the successive (random) time lengths between each positions in the WOS algorithm. Then, using (3.9), we have for all $a>0$

$$
\mathbb{P}\left(L_{1}|| x_{0} \mid=R+a\right)=\mathbb{P}\left(S_{N_{e}}\right)=\mathbb{E}\left(\prod_{i=0}^{N_{e}} \frac{r_{i} \bar{\kappa} / \sqrt{\varepsilon_{\mathrm{ext}}}}{\sinh \left(r_{i} \bar{\kappa} / \sqrt{\varepsilon_{\mathrm{ext}}}\right)}\right)=\mathbb{E}\left[\exp \left(-\frac{\bar{\kappa}^{2}}{2 \varepsilon_{\mathrm{ext}}} \sum_{i=0}^{N_{e}} t_{i}\right)\right] .
$$

Now, the positions $y_{i}$, the radii $r_{i}$ and the times $t_{i}$ satisfy, for some Brownian motion $\left(y_{0}+B_{t}, t \geq 0\right)$, for all $i \geq 0$,

$$
t_{i}=\inf \left\{t \geq 0: y_{0}+B_{t_{0}+\ldots+t_{i-1}+t} \in \partial S\left(y_{i}, r_{i}\right)\right\},
$$

with the convention $t_{-1}=0$. Therefore, by definition of $N_{e}$,

$$
\tau_{\Gamma+\varepsilon} \leq \sum_{i=0}^{N_{e}} t_{i} \leq \tau_{\Gamma}
$$


where $\tau_{\Gamma}=\inf \left\{t \geq 0: y_{0}+B_{t} \in \Gamma\right\}$ and $\tau_{\Gamma+\varepsilon}=\inf \left\{t \geq 0:\left|y_{0}+B_{t}\right|=R+\varepsilon\right\}$. Hence, using well-known results on first hitting times of the norm of a 3 -dimensional Brownian motion (see e.g. [4]),

$$
\frac{R}{R+a} \mathrm{e}^{-a \bar{\kappa} / \sqrt{\varepsilon_{\mathrm{ext}}}}=\mathbb{E}\left[\mathrm{e}^{-\frac{\bar{\kappa}^{2}}{2 \varepsilon_{\mathrm{ext}}} \tau_{\Gamma}}\right] \leq \mathbb{P}\left(L_{1}|| x_{0} \mid=R+a\right) \leq \mathbb{E}\left[\mathrm{e}^{-\frac{\bar{\kappa}^{2}}{2 \varepsilon} \tau_{\mathrm{ext}}+\varepsilon}\right]=\frac{R+\varepsilon}{R+a} \mathrm{e}^{-(a-\varepsilon) \bar{\kappa} / \sqrt{\varepsilon_{\mathrm{ext}}}}
$$

Therefore,

$$
\mathbb{P}\left(L_{1}|| x_{0} \mid=R+a\right)=1-\frac{\beta}{R} a+O\left(a^{2}+\varepsilon\right),
$$

which ends the proof of Theorem 4.1.

Theorem 4.1 has the following consequences.

Corollary 4.2. Assume that, for all $h>0$, random variables $A_{h}$ and $B_{h}$ are defined, that satisfy the assumptions of Theorem 4.1 and such that, for some $\alpha>0$,

$$
\begin{aligned}
& \mathbb{E}\left(A_{h} \mid A_{h}<0\right)=-h+O\left(h^{2}\right), \quad \mathbb{E}\left(A_{h} \mid A_{h}>0\right)=\alpha h+O\left(h^{2}\right), \\
& \mathbb{P}\left(A_{h}>0\right)=\frac{\varepsilon_{\text {ext }}}{\varepsilon_{\text {ext }}+\alpha \varepsilon_{\text {int }}}+O(h), \quad \mathbb{E}\left(A_{h}^{2}\right)=O\left(h^{2}\right), \quad \mathbb{E}\left(\left|A_{h}\right|^{3}\right)=O\left(h^{3}\right) \\
& \text { and } \mathbb{P}\left(B_{h}=0\right)=O\left(h^{2}\right)
\end{aligned}
$$

when $h \rightarrow 0$. Then, for all $x_{0} \in \Omega_{\text {int }}$,

$$
\begin{aligned}
\bar{u}_{\left(A_{h}, B_{h}\right)}\left(x_{0}\right)-\left(u-u_{0}\right)\left(x_{0}\right)=O\left(h^{2}\right. & +\varepsilon / h)-\frac{q\left(\alpha \varepsilon_{\text {int }}+\varepsilon_{\text {ext }}\right)}{4 \pi \varepsilon_{\text {ext }} \alpha \beta R}\left(\frac{\mathbb{E}\left(A_{h}^{+}\right)}{\varepsilon_{\text {ext }} h}-\frac{\mathbb{E}\left(A_{h}^{-}\right)}{\varepsilon_{\text {int }} h}\right)+\frac{q\left(\alpha \varepsilon_{\text {int }}+\varepsilon_{\text {ext }}\right)}{4 \pi \varepsilon_{\text {ext }} \alpha \beta^{2} R^{2}} \\
& \times\left(\frac{\beta}{\varepsilon_{\text {int }}} \frac{\mathbb{E}\left(\left(A_{h}^{-}\right)^{2}\right)}{h}+\frac{\beta+R^{2} \bar{\kappa}^{2} / 2 \varepsilon_{\text {ext }}}{\varepsilon_{\text {ext }}} \frac{\mathbb{E}\left(\left(A_{h}^{+}\right)^{2}\right)}{h}-\frac{R^{2}}{\varepsilon_{\text {ext }}} \frac{\mathbb{P}\left(B_{h}=0\right)}{h}\right)
\end{aligned}
$$

as $h \rightarrow 0$, where $a^{+}=a \vee 0$ and $a^{-}=(-a) \vee 0$ for all $a \in \mathbb{R}$. In particular, the bias $\bar{u}_{\left(A_{h}, B_{h}\right)}\left(x_{0}\right)-\left(u-u_{0}\right)\left(x_{0}\right)$ is $O(h+\varepsilon / h)$.

Corollary 4.3. When $\Omega_{\mathrm{int}}=S(0, R)$, for all $x_{0} \in \Omega_{\mathrm{int}}$,

$$
\begin{aligned}
& \bar{u}_{h}^{\operatorname{ANJ}(\alpha)}\left(x_{0}\right)-\left(u-u_{0}\right)\left(x_{0}\right)=\frac{q\left(1+\alpha\left(1+\frac{\bar{\kappa}^{2} R^{2}}{2 \varepsilon_{\mathrm{ext}} \beta}\right)\right)}{4 \pi R^{2} \beta \varepsilon_{\mathrm{ext}}} h+O\left(h^{2}+\varepsilon / h\right), \\
& \bar{u}_{h}^{\mathrm{UANJ}(\alpha)}\left(x_{0}\right)-\left(u-u_{0}\right)\left(x_{0}\right)=O(\varepsilon / h) \\
\text { and } \quad & \bar{u}_{h}^{\operatorname{NTJ}(\alpha)}\left(x_{0}\right)-\left(u-u_{0}\right)\left(x_{0}\right)=O\left(h^{2}+\varepsilon / h\right)
\end{aligned}
$$

as $h \rightarrow 0$. Note also that $\bar{u}_{h}^{\mathrm{SNJ}}=\bar{u}_{h}^{\mathrm{ANJ}(1)}$.

Remark 4.4. If $\hat{u}_{h}^{\mathrm{NTJ}(\alpha)}\left(x_{0}\right)$ denotes the expected score of the NTJ algorithm without killing (i.e. without Step (2c)), a similar computation would lead to

$$
\hat{u}_{h}^{\mathrm{NTJ}(\alpha)}\left(x_{0}\right)-\left(u-u_{0}\right)\left(x_{0}\right)=\frac{q \bar{\kappa}^{2}}{3 \pi \beta^{2} \varepsilon_{\mathrm{int}} \varepsilon_{\mathrm{ext}}} h+O\left(h^{2}+\varepsilon / h\right) .
$$

This shows that, in the case of a single sphere, adding the killing step in the NTJ algorithm improves the order of the algorithm. Since this additional killing step also reduces the lifetime of the particle and hence the computational cost, there is no interest to omit this step in the NTJ algorithm. 
Proof of Corollary 4.2. Taylor expansions and the explicit formula (4.1) lead to

$$
\begin{aligned}
\mathbb{E}\left[B_{h} w\left(R+A_{h}\right)\right]-w(R)=-w & (R) \mathbb{E}\left(1-B_{h}\right)+\mathbb{E}\left[B_{h} A_{h}\left(w^{\prime}(R-) \mathbb{1}_{A_{h}<0}+w^{\prime}(R+) \mathbb{1}_{A_{h}>0}\right)\right] \\
& +\frac{1}{2} \mathbb{E}\left[B_{h} A_{h}^{2}\left(w^{\prime \prime}(R-) \mathbb{1}_{A_{h}<0}+w^{\prime \prime}(R+) \mathbb{1}_{A_{h}>0}\right)\right]+O\left(\mathbb{E}\left(A^{3}\right)\right) \\
=- & \frac{q \mathbb{P}\left(B_{h}=0\right)}{4 \pi \varepsilon_{\text {ext }} \beta R}-\frac{q}{4 \pi R^{2}} \mathbb{E}\left[B_{h} A_{h}\left(\frac{\mathbb{1}_{A_{h}<0}}{\varepsilon_{\text {int }}}+\frac{\mathbb{1}_{A_{h}>0}}{\varepsilon_{\text {ext }}}\right)\right] \\
& +\frac{q}{4 \pi R^{3}} \mathbb{E}\left[B_{h} A_{h}^{2}\left(\frac{\mathbb{1}_{A_{h}<0}}{\varepsilon_{\text {int }}}+\frac{\mathbb{1}_{A_{h}>0}}{\varepsilon_{\text {ext }}}+\frac{\bar{\kappa}^{2} R^{2} \mathbb{1}_{A_{h}>0}}{2 \varepsilon_{\text {ext }}^{2} \beta}\right)\right]+O\left(h^{3}\right) .
\end{aligned}
$$

Because of our assumption on $A_{h}$, the term of order $h$ in the r.h.s. vanishes, and thus this r.h.s. is $O\left(h^{2}\right)$. Moreover,

$$
\mathbb{P}\left(B_{h}=0\right)+\frac{\beta}{R} \mathbb{E}\left(B_{h} A_{h} \mathbb{1}_{A_{h}>0}\right)=\frac{\alpha \beta \varepsilon_{\text {ext }}}{R\left(\varepsilon_{\text {ext }}+\alpha \varepsilon_{\text {int }}\right)} h+O\left(h^{2}\right) .
$$

Combining these estimates with (4.3) yields (4.9).

Proof of Corollary 4.3. In the case of a single sphere, the $\operatorname{SNJ}, \operatorname{ANJ}(\alpha)$ and $\operatorname{UANJ}(\alpha)$ algorithms correspond to random variables $A_{h}$ and $B_{h}$ in the generic algorithm that obviously satisfy the assumptions of Corollary 4.2. The only non-trivial point is to prove the $\mathbb{P}\left(B_{h}=0\right)=O\left(h^{2}\right)$ in the UANJ algorithm, but it can be easily checked from (3.13) and (3.14) that, in this case,

$$
\mathbb{P}\left(B_{h}=0\right)=1-\mathbb{E}\left(\exp \left(-\bar{\kappa}^{2} \tau_{h, \alpha}^{\mathrm{ext}}\right)\right)=\frac{\alpha^{2} \bar{\kappa}^{2}}{2\left(\alpha \varepsilon_{\mathrm{int}}+\varepsilon_{\mathrm{ext}}\right)} h^{2}+O\left(h^{3}\right) .
$$

The bias (4.10) of the $\operatorname{ANJ}(\alpha)$ algorithm then follows from easy computations. In the UANJ $(\alpha)$ algorithm, the corresponding random variables $A_{h}$ and $B_{h}$ satisfy

$$
w(R)=\mathbb{E}\left[B_{h} w\left(R+A_{h}\right)\right]
$$

by construction and Feynman-Kac's formula. Therefore, (4.11) follows from (4.3) and calculations of the proof of Corollary 4.2.

The NTJ algorithm also satisfies the assumptions of Corollary 4.2, but calculations are more difficult. Letting $V$ be a uniform random vector on $S^{2}$ and $E$ an independent exponential random variable of parameter 1 , we have

$$
A_{h} \stackrel{(d)}{=} \begin{cases}|(R, 0,0)+2 h E V|-R=\left(R^{2}+4 h R E V_{1}+4 h^{2} E^{2}\right)^{1 / 2}-R & \text { if } V_{1}<0 \\ |(R, 0,0)+2 \alpha h E V|-R=\left(R^{2}+4 \alpha h R E V_{1}+4 \alpha^{2} h^{2} E^{2}\right)^{1 / 2}-R & \text { if } V_{1} \geq 0\end{cases}
$$

and

$$
\mathbb{P}\left(B_{h}=0\right)=1-\mathbb{P}\left(V_{1}<0\right)-\mathbb{E}\left(\exp \left(-\frac{4 \bar{\kappa}^{2} \alpha h^{2}}{3 \varepsilon_{\mathrm{int}}} E\right) \mathbb{1}_{V_{1}>0}\right)=\frac{2 \bar{\kappa}^{2} \alpha}{3 \varepsilon_{\mathrm{int}}} h^{2}+O\left(h^{4}\right) .
$$

Expanding w.r.t. $h$, we have

$$
A_{h}=2 E h\left[-V_{1}^{-}+\alpha V_{1}^{+}+\frac{h E}{R}\left(1-V_{1}^{2}\right)\left(\mathbb{1}_{V_{1}<0}+\alpha^{2} \mathbb{1}_{V_{1}>0}\right)\right]+O\left(h^{3} E^{3}\right)
$$

where $\alpha=\varepsilon_{\text {ext }} / \varepsilon_{\text {int }}$. In particular, $A_{h}>0$ iff $V_{1}>0$ or $V_{1}<0$ and $\left|V_{1}\right|<f\left(h E, V_{1}\right)$, where the function $f$ satisfies

$$
f\left(h E, V_{1}\right)=\frac{h E}{R}\left(1-V_{1}^{2}\right)+O\left(h^{2} E^{2}\right) \leq \frac{h E}{R}\left(1-V_{1}^{2}+C h E\right),
$$


for some constant $C>0$. Considering the r.h.s. above as a polynomial in $h E$, one can easily see that $\left|V_{1}\right|<$ $f\left(h E, V_{1}\right)$ implies that $h E>g\left(\left|V_{1}\right|\right)$, where

$$
g\left(\left|V_{1}\right|\right)=\frac{1-V_{1}^{2}+\sqrt{\left(1-V_{1}^{2}\right)+4 C R\left|V_{1}\right|}}{2 C} \geq \frac{R\left|V_{1}\right|}{\sqrt{1+4 C R}}
$$

since $\sqrt{a^{2}+b}-a \geq b / \sqrt{\bar{a}^{2}+\bar{b}}$ for all $a \in[0, \bar{a}]$ and $b \in[0, \bar{b}]$. It then follows from elementary computations that

$$
\begin{aligned}
\mathbb{E}\left(A_{h} \mathbb{1}_{A_{h}<0}\right) & =\mathbb{E}\left(A_{h} \mathbb{1}_{V_{1}<0}\right)+\mathbb{E}\left(A_{h} \mathbb{1}_{V_{1}<0,\left|V_{1}\right|<f\left(h E, V_{1}\right)}\right) \\
& =-\frac{h}{2}+\frac{4 h^{2}}{3 R}+O\left(h^{3}\right)+O\left(\mathbb{E}\left[\left(h E\left|V_{1}\right|+h^{2} E^{2}\right) \mathbb{1}_{h E>g\left(\left|V_{1}\right|\right)}\right]\right),
\end{aligned}
$$

where the last term comes from (4.13) and the fact that $|\sqrt{1+a}-1| \leq|a|$ for all $a \geq-1$. Now, observing that, in spherical coordinates, $V_{1}=\cos (\Phi)$ where $\Phi$ has law $\frac{\sin \varphi}{2} \mathbb{1}_{\varphi \in[0, \pi]} \mathrm{d} \varphi$,

$$
\begin{aligned}
\mathbb{E}\left[E\left|V_{1}\right| \mathbb{1}_{h E>g\left(\left|V_{1}\right|\right)}\right] & =\mathbb{E}\left[\left|V_{1}\right|\left(\frac{g\left(\left|V_{1}\right|\right)}{h}+1\right) \mathrm{e}^{-g\left(\left|V_{1}\right|\right) / h}\right] \leq C^{\prime} \mathbb{E}\left[\left|V_{1}\right| \mathrm{e}^{-g\left(\left|V_{1}\right|\right) / 2 h}\right] \\
& \leq C^{\prime} \int_{0}^{\pi / 2} \sin \varphi \cos \varphi \mathrm{e}^{-R \cos \varphi / \sqrt{1+4 C R} h} \mathrm{~d} \varphi \leq \frac{1+4 C R}{R^{2}} h^{2} .
\end{aligned}
$$

Similarly,

$$
\begin{aligned}
\mathbb{E}\left[E^{2} \mathbb{1}_{V_{1}<0,\left|V_{1}\right|<f\left(h E, V_{1}\right)}\right] & =O(h), \\
\mathbb{P}\left(V_{1}<0,\left|V_{1}\right|<f\left(h E, V_{1}\right)\right) & =O(h) .
\end{aligned}
$$

In view of (4.14), we thus have

Since

$$
\mathbb{E}\left(A_{h} \mathbb{1}_{A_{h}<0}\right)=-\frac{h}{2}+\frac{4 h^{2}}{3 R}+O\left(h^{3}\right)
$$

$$
\mathbb{E} A_{h}=\frac{h}{2}+\frac{h \alpha}{2}+\frac{h^{2}}{3 R}+\frac{\alpha h^{2}}{3 R}+O\left(h^{3}\right),
$$

we deduce that

Similarly, it can be checked that

$$
\mathbb{E}\left(A_{h} \mathbb{1}_{A_{h}>0}\right)=\frac{\alpha h}{2}+\frac{4 \alpha^{2} h^{2}}{3 R}+O\left(h^{3}\right)
$$

and

$$
\mathbb{E}\left(A_{h}^{2} \mathbb{1}_{A_{h}<0}\right)=\frac{4 h^{2}}{3}+O\left(h^{3}\right)
$$

$$
\mathbb{E}\left(A_{h}^{2} \mathbb{1}_{A_{h}>0}\right)=\frac{4 \alpha^{2} h^{2}}{3}+O\left(h^{3}\right) .
$$

In conclusion, the NTJ algorithm satisfies the assumptions of Corollary 4.2, and (4.9) combined with the previous estimates yields (4.12).

Note that, when $V_{1}$ is negative, $A_{h}$ has some probability to be positive (i.e. the particle could exit from $\Omega_{\text {int }}$ ), in which case the neutron transport interpretation of the algorithm would suggest to modify the algorithm, for example by adding a probability of killing depending on the time spent by the particle in $\Omega_{\text {ext }}$. However, as can be checked from the previous calculation, such a modification would not change the bias estimate (4.12), so we chose to present the simplest version of the NTJ algorithm. 
To conclude this study of the bias in the case of one sphere, let us compute the optimal (in terms of bias) distribution for $A_{h}$, under the assumptions of Corollary 4.2 , when $\alpha$ is fixed, $B_{h}=1$ a.s. and $\mathbb{P}\left(A_{h}>0\right)=$ $\varepsilon_{\text {ext }} /\left(\alpha \varepsilon_{\text {int }}+\varepsilon_{\text {ext }}\right)$ for all $h>0$ (which is the case is the SNJ and ANJ algorithms). In this case, the leading term of the bias in (4.9) is proportional to

$$
\begin{aligned}
\alpha \frac{\mathbb{E}\left(A^{2} \mid A<0\right)}{h}+\frac{\mathbb{E}\left(A^{2} \mid A>0\right)}{h}\left(1+\frac{\bar{\kappa}^{2} R^{2}}{2 \varepsilon_{\text {ext }} \beta}\right) & \\
= & \alpha \frac{\operatorname{Var}(A \mid A<0)+h^{2}}{h}+\frac{\operatorname{Var}(A \mid A>0)+\alpha^{2} h^{2}}{h}\left(1+\frac{\bar{\kappa}^{2} R^{2}}{2 \varepsilon_{\text {ext }} \beta}\right) .
\end{aligned}
$$

which is minimal if $A$ is constant conditionally on $\{A>0\}$ and $\{A<0\}$, i.e. in the ANJ case.

When $\alpha$ is not fixed, the optimal value for $\alpha$ in terms of bias is of course $\alpha=0$ (with the limitation in the WOS algorithm that $\alpha h>\varepsilon$ ). However, this is clearly not optimal in terms of the computational cost, since, when $\alpha$ decreases, the walk on spheres outside of the molecule becomes very costly and must be repeated an increasing number of times before the particle gets killed. The next section is devoted to an estimation of the computational cost of the algorithm in the case of one sphere.

\subsubsection{Estimation of the computational cost}

Theorem 4.5. Assume that $\Omega_{\mathrm{int}}=S(0, R)$ with $R>0$. Let $N_{\mathrm{e}}^{\varepsilon, R_{0}}$ denote the number of steps in the WOS algorithm with killing rate $\lambda=\bar{\kappa}^{2} / 2 \varepsilon_{\text {ext }}$, starting from $y_{0} \in \Omega_{\text {ext }}$ at a distance $R_{0}$ from $\Gamma$ and with threshold $\varepsilon$. With the notation $\mu_{\mathrm{ext}}=\bar{\kappa} / \sqrt{\varepsilon_{\mathrm{ext}}}$, we have

$$
\mathbb{E}\left(N_{\mathrm{e}}^{\varepsilon, R_{0}}\right) \leq \frac{\left[(2 R+\varepsilon) \vee\left(2 / \mu_{\mathrm{ext}}\right)\right] R_{0}}{\left(R+R_{0}\right) \sinh \left(\mu_{\mathrm{ext}} R_{0}\right)}\left(\frac{1}{2(1-\log 2)}+\frac{\log 2}{2(1-\log 2)^{3}}+\frac{\log \left(R_{0} / \varepsilon\right)}{2(1-\log 2)^{2}}\right) .
$$

Proof. The proof is based on the estimate in [32] of the expected cost of the WOS algorithm in a half space.

Let us first compute the law of the distance to $\Gamma$ of the next position $y_{1}$ in the WOS algorithm. The largest tangent sphere to $\Gamma$ has radius $R_{0}$, and the angle $\Theta$ between the vectors $y_{1}-y_{0}$ and $y_{0}$ has distribution

$$
\frac{\sin \theta}{2} \mathbb{1}_{\theta \in[0, \pi]} \mathrm{d} \theta .
$$

The distance $R_{1}$ between $y_{1}$ and $\Gamma$ is $\sqrt{R^{2}+2 R_{0}\left(R+R_{0}\right)(1-\cos \Theta)}$, and its density is

$$
\frac{r}{2 R_{0}\left(R_{0}-r\right)} \mathbb{1}_{r \in\left[0,2 R_{0}\right]}
$$

Let $\left(R_{k}\right)_{k \geq 1}$ denote the sequence of radii in the WOS algorithm (without killing). We then have for all $k \geq 2$,

$$
\begin{aligned}
\mathbb{P}\left(N_{\mathrm{e}}^{\varepsilon, R_{0}} \geq k\right) & =\int_{\varepsilon}^{2 R_{0}} \frac{R+R_{1}}{2 R_{0}\left(R+R_{0}\right)} \frac{\mu_{\text {ext }} R_{0}}{\sinh \left(\mu_{\text {ext }} R_{0}\right)} \mathrm{d} R_{1} \ldots \int_{\varepsilon}^{2 R_{k-2}} \frac{R+R_{k-1}}{2 R_{k-2}\left(R+R_{k-2}\right)} \frac{\mu_{\text {ext }} R_{k-2}}{\sinh \left(\mu_{\text {ext }} R_{k-2}\right)} \mathrm{d} R_{k-1} \\
& =\frac{1}{2^{k} \mu_{\text {ext }}\left(r+r_{0}\right) \sinh r_{0}} \int_{\gamma}^{2 r_{0}} \frac{\mathrm{d} r_{1}}{\sinh r_{1}} \ldots \int_{\gamma}^{2 r_{k-3}} \frac{\mathrm{d} r_{k-2}}{\sinh r_{k-2}} \int_{\gamma}^{2 r_{k-2}}\left(r+r_{k-1}\right) \mathrm{d} r_{k-1},
\end{aligned}
$$

where $r=\mu_{\mathrm{ext}} R, r_{0}=\mu_{\mathrm{ext}} R_{0}$ and $\gamma=\mu_{\mathrm{ext}} \varepsilon$.

Now, fix $a>0$ and let $\varphi(x)=x(a+x) / \sinh x$ for $x>0$. The function $\varphi$ has a unique maximum on $(0,+\infty)$ at the unique solution $x^{*}$ of $\tanh x=x(a+x) /(a+2 x)$. Therefore, the maximal value of $\varphi$ is

$$
\left(a+2 x^{*}\right) / \cosh x^{*} \leq\left(2 a+4 x^{*}\right) /\left(2+\left(x^{*}\right)^{2}\right) .
$$


As a function of $x^{*}$, this quantity is maximal at the unique positive solution $x^{* *}$ of $\left(x^{*}\right)^{2}+a x^{*}-2=0$. Therefore, the maximal value of $\left(2 a+4 x^{*}\right) /\left(2+\left(x^{*}\right)^{2}\right)$ is equal to

$$
\frac{2}{x^{* *}}=\frac{a}{2}\left(1+\sqrt{1+\frac{8}{a^{2}}}\right) .
$$

This quantity is less than $2 a$ if $a \geq 1$, and less than 2 otherwise. In conclusion, we have

$$
\frac{x(a+x)}{\sinh x} \leq 2(a \vee 1) \quad \forall x \geq 0 .
$$

Combining this with (4.16), we get

$$
\mathbb{P}\left(N_{\mathrm{e}}^{\varepsilon, R_{0}} \geq k\right) \leq \frac{[(2 r+\gamma) \vee 2] r_{0}}{\mu_{\mathrm{ext}}\left(r+r_{0}\right) \sinh r_{0}} \mathbb{P}^{\text {hyp }}\left(N_{\mathrm{e}}^{\gamma, r_{0}} \geq k\right),
$$

where

$$
\mathbb{P}^{\text {hyp }}\left(N_{\mathrm{e}}^{\gamma, r_{0}} \geq k\right)=\frac{1}{2^{k-1} r_{0}} \int_{\gamma}^{2 r_{0}} \frac{\mathrm{d} r_{1}}{r_{1}} \cdots \int_{\gamma}^{2 r_{k-3}}\left(2 r_{k-2}-\gamma\right) \frac{\mathrm{d} r_{k-2}}{r_{k-2}}
$$

is the probability that the WOS algorithm without killing, when $\Gamma$ is a hyperplane and started at a distance $r_{0}$ from $\Gamma$, has never reached a position at a distance less than $\gamma$ from $\Gamma$ after $k-1$ steps. Hence,

$$
\mathbb{E}\left(N_{\mathrm{e}}^{\varepsilon, R_{0}}\right) \leq \frac{[(2 r+\gamma) \vee 2] r_{0}}{\mu_{\mathrm{ext}}\left(r+r_{0}\right) \sinh r_{0}} \mathbb{E}^{\mathrm{hyp}}\left(N_{\mathrm{e}}^{\gamma, r_{0}}\right) .
$$

Sabelfeld and Talay [32] have computed an explicit bound on the expectation of $N_{\mathrm{e}}^{\gamma, r_{0}}$ under $\mathbb{P}^{\text {hyp }}$ which leads to $(4.15)$.

The previous result allows one to bound from above the mean computational cost of our algorithms in the case of one sphere. We only give details in the $\operatorname{ANJ}(\alpha)$ case.

Corollary 4.6. For any $\alpha>0$, the mean computational cost of the $A N J(\alpha)$ algorithm when $\Omega_{\mathrm{int}}=S(0, R)$, expressed in units of the time length of a single step of the WOS algorithm (assume to be equal to the time length of a single step of the UWOS algorithm), is bounded from above by

$$
2 \frac{R \vee \mu_{\mathrm{ext}}^{-1}}{\alpha \beta \mu_{\mathrm{ext}} h}\left(C_{1}+C_{2} \log \frac{\alpha h}{\varepsilon}\right)+\frac{R \varepsilon_{\mathrm{int}}}{\beta \varepsilon_{\mathrm{ext}} h}+O\left(1+\varepsilon \log (1 / \varepsilon) / h^{2}\right),
$$

where $C_{1}=(1-\log 2)^{-1}+\log 2(1-\log 2)^{-3}$ and $C_{2}=(1-\log 2)^{-2}$.

Proof. At each time where the particle jumps from $\Gamma$ to $\Omega_{\text {ext }}$, the probability that the particle hits $\Gamma$ again before getting extinct is given by (4.8). Therefore, the number of times the particle jumps from $\Gamma$ to $\Omega_{\text {ext }}$ before being killed is a geometric random variable $G$ with expectation $R / \alpha \beta h+O\left(1+\varepsilon / h^{2}\right)$. Conditionally on the value of $G$, the number of times where the particle jumps from $\Gamma$ to $\Omega_{\text {int }}$ before being killed is

$$
N=1+\sum_{i=1}^{G}\left(N_{i}-1\right),
$$

where $N_{i}$ are i.i.d. r.v. with geometric distribution with parameter $\varepsilon_{\text {ext }} /\left(\alpha \varepsilon_{\text {int }}+\varepsilon_{\text {ext }}\right)$.

Denoting by $L$ the event "the particle is alive at the end of the WOS algorithm", it follows from (4.8) and Theorem 4.5 that

$$
\mathbb{E}\left(N_{\mathrm{e}}^{\varepsilon, \alpha h} \mid L\right) \leq \frac{R \vee \mu_{\mathrm{ext}}^{-1}}{R \mu_{\mathrm{ext}}}\left(C_{1}+C_{2} \log \frac{\alpha h}{\varepsilon}\right)(1+O(h+\varepsilon))
$$


and

$$
\mathbb{E}\left(N_{\mathrm{e}}^{\varepsilon, \alpha h} \mid L^{c}\right) \leq \frac{R \vee \mu_{\mathrm{ext}}^{-1}}{\alpha \beta \mu_{\mathrm{ext}} h}\left(C_{1}+C_{2} \log \frac{\alpha h}{\varepsilon}\right)(1+O(h+\varepsilon / h)) .
$$

Now, the mean computational cost is given by

$$
\mathbb{E}\left[N+(G-1) \mathbb{E}\left(N_{\mathrm{e}}^{\varepsilon, \alpha h} \mid L\right)+\mathbb{E}\left(N_{\mathrm{e}}^{\varepsilon, \alpha h} \mid L^{c}\right)\right],
$$

which, combined with previous estimates, gives (4.17).

From this result and the estimation of the bias of the ANJ algorithm (4.10), one gets that the leading-order term in the efficiency of the algorithm (mean computational cost times mean bias) is bounded by

$$
\left(2 \frac{R \vee \mu_{\text {ext }}^{-1}}{\alpha \beta \mu_{\mathrm{ext}}}\left(C_{1}+C_{2} \log \frac{\alpha h}{\varepsilon}\right)+\frac{R \varepsilon_{\mathrm{int}}}{\beta \varepsilon_{\mathrm{ext}}}\right) \frac{q\left(1+\alpha\left(1+\frac{\bar{\kappa}^{2} R^{2}}{2 \varepsilon_{\mathrm{ext}} \beta}\right)\right)}{4 \pi R^{2} \beta \varepsilon_{\mathrm{ext}}} .
$$

This quantity is minimal if $\alpha$ satisfies

$$
A B \alpha^{2}+A \alpha+1=\log \frac{\alpha h}{\varepsilon}+\frac{C_{1}}{C_{2}},
$$

where

$$
A=1+\frac{\bar{\kappa}^{2} R^{2}}{2 \varepsilon_{\mathrm{ext}} \beta} \quad \text { and } \quad B=\frac{\varepsilon_{\mathrm{int}} \mu_{\mathrm{ext}}}{2 \varepsilon_{\mathrm{ext}} C_{2}\left(1 \vee\left(\mu_{\mathrm{ext}} R\right)^{-1}\right)} .
$$

Denoting by $\alpha^{*}(\varepsilon, h)$ the value of $\alpha$ that minimizes (4.18), this suggests to introduce another version of the ANJ algorithm, that we call Optimized ANJ (OANJ) algorithm, defined as the $\operatorname{ANJ}\left(\alpha^{*}(\varepsilon, h)\right)$ algorithm, which, as the other algorithms presented above, will be tested in Section 5.

\subsection{General case}

In this section, we extend the bias estimates of the previous sections to situations where $\Omega_{\text {int }}$ is not a sphere. Since our method is based on uniform bounds on the second-order derivatives of the solution $u$ of PoissonBoltzmann equation, our result only holds in situations where we can prove that $\nabla^{2} u$ is bounded, i.e. if $\Gamma$ is $C^{\infty}$ by Theorem 2.17. Note that, in this case, the UWOS algorithm inside $\Omega_{\text {int }}$ must be replaced by the WOS algorithm.

We consider a family of random variables $\left(A_{x}, B_{x}\right)_{x \in \Gamma}$ with $A_{x} \in \mathbb{R}^{3}$ and $B_{x} \in\{0,1\}$, and let $\left(A_{x}^{k}, B_{x}^{k}\right)_{k \geq 1}$ be i.i.d. copies of $\left(A_{x}, B_{x}\right)$ for all $x \in \Gamma$. We construct the following algorithm.

\section{Generalized generic algorithm.}

This algorithm is the SNJ algorithm where Step (2) is replaced by

(2) Let $x_{k+1}=\operatorname{exit}\left(x_{k}\right)+A_{\text {exit }\left(x_{k}\right)}^{k}$ and the particle is killed if $B_{\text {exit }\left(x_{k}\right)}^{k}=0$.

We denote by $\bar{u}_{\left(A_{x}, B_{x}\right)_{x}}\left(x_{0}\right)$ the expectation of the score given by this algorithm started from $x_{0} \in \mathbb{R}^{3}$.

Theorem 4.7. Assume that $\Gamma$ is a $C^{\infty}$ compact submanifold of $\mathbb{R}^{3}$. With the previous notation, we have

$$
\begin{aligned}
& \sup _{x_{0} \in \Omega_{\mathrm{int}}}\left|\bar{u}_{\left(A_{x}, B_{x}\right)_{x}}\left(x_{0}\right)-\left(u-u_{0}\right)\left(x_{0}\right)\right| \\
& \quad \leq \sup _{x \in \Gamma} \frac{\left|\mathbb{E}\left[B_{x} u\left(x+A_{x}\right)\right]-u(x)\right|+\varepsilon\|\nabla u\|_{L^{\infty}\left(\mathbb{R}^{3}\right)}}{1-\mathbb{E}\left[B_{x}\left(\mathbb{1}_{\left\{x+A_{x} \in \Omega_{\mathrm{int}} \cup \Gamma\right\}}+\mathbb{1}_{\left\{x+A_{x} \in \Omega_{\mathrm{ext}}\right\}}\left(L_{1} \mid x_{0}=x+A_{x}\right)\right)\right]} .
\end{aligned}
$$


In the case of all the algorithms described in Section 3, we actually have

$$
\sup _{x_{0} \in \Omega_{\mathrm{int}}}\left|\bar{u}_{h}^{\text {algo }}\left(x_{0}\right)-\left(u-u_{0}\right)\left(x_{0}\right)\right|=O(h+\varepsilon / h),
$$

where algo $\in\{S N J, A N J(\alpha), U A N J(\alpha), O A N J, N T J\}$ for any $\alpha>0$.

Remark 4.8. The computations of Section 4.1.1 suggest that the bias of the neutron transport algorithm could be of order $h^{2}+\varepsilon / h$. But this result is due to the fact that the first-order correction in the probability of exit from $\Gamma$, the second-order correction in the expected distance from $\Gamma$, and the second-order expansion of $\mathbb{E}\left[u\left(x+A_{x}\right)\right]$, exactly cancel in the case of one sphere. It is unclear whether this holds in the more general case of Theorem 4.7, particularly because nothing is known a priori on the second-order interior and exterior normal derivatives of $u$ on $\Gamma$.

Concerning the $\operatorname{UANJ}(\alpha)$ algorithm, its bias is certainly not of a smaller order than $h+\varepsilon / h$, since it only allows jumps from $\Gamma$ in the normal direction. Indeed, errors should occur, due to the fact that the process $X$ also moves in the tangential directions from $\Gamma$, to distances of order $h$ (since $X$ moves of a distance of order $h$ in the normal direction), which should contribute to a nonzero error in the second-order terms in the expansion of the numerator in (4.19).

The proof of the previous result easily extends to any dimension $d$ and to any solution to the PDE (2.42) which satisfy the assumptions of Theorem 2.17. In such situation, all the algorithms we describe have a bias of order $h+\varepsilon / h$.

Proof. The proof of (4.19) closely follows the proof of (4.3) in Theorem 4.1. First, the formula (4.5) trivially extends to the previous generalized algorithm. Now, since the simulation of exit $\left(x_{0}\right)$ by the UWOS algorithm is exact if $x_{0} \in \Omega_{\text {int }}$, we deduce from (3.5) that, for all $x_{0} \in \Omega_{\text {int }}$,

$$
\bar{u}_{\left(A_{x}, B_{x}\right)_{x}}\left(x_{0}\right)-\left(u-u_{0}\right)\left(x_{0}\right)=\mathbb{E}\left[\bar{u}\left(\operatorname{exit}\left(x_{0}\right)\right)-u\left(\operatorname{exit}\left(x_{0}\right)\right)+u_{0}\left(\operatorname{exit}\left(x_{0}\right)\right)\right] .
$$

As in the proof of Theorem 4.1, we then obtain that, for all $x_{0} \in \Gamma$,

$$
\begin{aligned}
\bar{u}(x) & -\left(u-u_{0}\right)(x)=\mathbb{E}\left[B_{x} \mathbb{1}_{\left\{x+A_{x} \in \Omega_{\mathrm{int}} \cup \Gamma\right\}}\left(u_{0}\left(x+A_{x}\right)+\bar{u}\left(x+A_{x}\right)\right)\right] \\
& +\mathbb{E}\left[B_{x} \mathbb{1}_{\left\{x+A_{x} \in \Omega_{\mathrm{ext}}\right\}} \bar{u}\left(x+A_{x}\right)\right]-u(x) \\
=\mathbb{E} & {\left[B_{x} \mathbb{1}_{\left\{x+A_{x} \in \Omega_{\mathrm{int}} \cup \Gamma\right\}} \mathbb{E}\left[\bar{u}\left(\operatorname{exit}\left(x_{0}\right)\right)-\left(u-u_{0}\right)\left(\operatorname{exit}\left(x_{0}\right)\right) \mid x_{0}=x+A_{x}\right]\right] } \\
& +\mathbb{E}\left[B_{x} \mathbb{1}_{\left\{x+A_{x} \in \Omega_{\mathrm{ext}}\right\}} \mathbb{E}\left[\mathbb{1}_{L_{1}}\left(\bar{u}\left(\operatorname{exit}\left(x_{0}\right)\right)-\left(u-u_{0}\right)\left(\operatorname{exit}\left(x_{0}\right)\right)\right) \mid x_{0}=x+A_{x}\right]\right] \\
& +\mathbb{E}\left[B_{x} \mathbb{1}_{\left\{x+A_{x} \in \Omega_{\mathrm{int}} \cup \Gamma\right\}} u\left(x+A_{x}\right)\right] \\
& +\mathbb{E}\left[B_{x} \mathbb{1}_{\left\{x+A_{x} \in \Omega_{\mathrm{ext}}\right\}} \mathbb{E}\left[\mathbb{1}_{L_{1}} u\left(\operatorname{exit}\left(x_{0}\right)\right) \mid x_{0}=x+A_{x}\right]\right]-u(x),
\end{aligned}
$$

where $\bar{u}=\bar{u}_{\left(A_{x}, B_{x}\right)_{x}}$. Since

$$
u\left(y_{0}\right)=\mathbb{E}\left[u\left(\operatorname{exit}\left(y_{0}\right)\right) \mathbb{1}_{L_{1}}\right]+O(\varepsilon)
$$

by (3.10), where the $O(\varepsilon)$ is bounded by $\varepsilon\|\nabla u\|_{L^{\infty}\left(\mathbb{R}^{3}\right)}$, we deduce (4.19) from (4.21) and (4.22).

Now, in all the algorithms of Section 3 ,

$$
\mathbb{E}\left[A_{x}^{2}\right]=O\left(h^{2}\right) \quad \text { and } \quad \mathbb{P}\left(B_{x}=0\right)=O\left(h^{2}\right) .
$$


Therefore, as in the proof of Corollary 4.2, it follows from a second-order expansion in the numerator of (4.19) and from the fact that $\left\|\nabla^{2} u\right\|_{L^{\infty}\left(\mathbb{R}^{3}\right)}<\infty$ that, for all $x_{0} \in \Omega_{\text {int }}$,

$$
\begin{aligned}
&\left|\bar{u}_{h}^{\text {algo }}\left(x_{0}\right)-\left(u-u_{0}\right)\left(x_{0}\right)\right| \\
& \leq \sup _{x \in \Gamma} \frac{\mathbb{E}\left[\nabla^{\mathrm{int}} u(x) \cdot A_{x} \mathbb{1}_{\left\{x+A_{x} \in \Omega_{\mathrm{int}}\right\}}+\nabla^{\mathrm{ext}} u(x) \cdot A_{x} \mathbb{1}_{\left\{x+A_{x} \in \Omega_{\mathrm{ext}}\right\}}\right]+O\left(h^{2}+\varepsilon\right)}{\mathbb{E}\left[\mathbb{1}_{\left\{x+A_{x} \in \Omega_{\mathrm{ext}}\right\}} \mathbb{P}\left(L_{1}^{c} \mid x_{0}=x+A_{x}\right)\right]+O\left(h^{2}\right)} .
\end{aligned}
$$

All our algorithms satisfy

$$
\mathbb{E}\left[\nabla^{\mathrm{int}} u(x) \cdot A_{x} \mathbb{1}_{\left\{x+A_{x} \in \Omega_{\mathrm{int}}\right\}}\right]=\nabla^{\mathrm{int}} u(x) \cdot n(x) \mathbb{E}\left[A_{x} \cdot n(x) \mathbb{1}_{\left\{x+A_{x} \in \Omega_{\mathrm{int}}\right\}}\right],
$$

and

$$
\varepsilon_{\text {ext }} \mathbb{E}\left[A_{x} \cdot n(x) \mathbb{1}_{\left\{x+A_{x} \in \Omega_{\text {int }}\right\}}\right]+\varepsilon_{\text {int }} \mathbb{E}\left[A_{x} \cdot n(x) \mathbb{1}_{\left\{x+A_{x} \in \Omega_{\text {ext }}\right\}}\right]=O\left(h^{2}\right),
$$

which shows that the numerator in $(4.23)$ is $O\left(h^{2}+\varepsilon\right)$.

Concerning the denominator, since $\Gamma$ is $C^{\infty}$ and compact, there exists $r>0$ such that, for all $x \in \Gamma$, the exterior sphere of radius $r$ tangent to $\Gamma$ at $x$ does not intersect $\Gamma$ except at $x$. Let us call $S_{x}$ the sphere with the same center as the previous one with radius $r-\varepsilon$ (assuming without loss of generality that $\varepsilon<r$ ). By the standard coupling of the WOS algorithm with a Brownian motion $\left(B_{t}, t \geq 0\right)$ in $\mathbb{R}^{3}$, for all $x \in \Omega_{\text {ext }}$ such that $\varepsilon<\mathrm{d}(x, \Gamma) \leq r$

$$
\begin{aligned}
\mathbb{P}\left(L_{1}^{c} \mid x_{0}=x\right) & \geq 1-\mathbb{E}\left[\exp \left(-\frac{\bar{\kappa}^{2}}{2 \varepsilon_{\text {ext }}} \tau_{x}\right)\right]=1-\frac{(r-\varepsilon) \sinh \left((r-\mathrm{d}(x, \Gamma)) \bar{\kappa} / \sqrt{\varepsilon_{\text {ext }}}\right)}{(r-\mathrm{d}(x, \Gamma)) \sinh \left((r-\varepsilon) \bar{\kappa} / \sqrt{\varepsilon_{\text {ext }}}\right)} \\
& \geq 1-\frac{r \sinh \left((r-\mathrm{d}(x, \Gamma)) \bar{\kappa} / \sqrt{\varepsilon_{\text {ext }}}\right)}{(r-\mathrm{d}(x, \Gamma)) \sinh \left(r \bar{\kappa} / \sqrt{\varepsilon_{\text {ext }}}\right)}
\end{aligned}
$$

where $\tau_{x}=\inf \left\{t \geq 0: x+B_{t} \notin S_{\pi(x)}\right\}$. We may assume without loss of generality that $\varepsilon \leq h \eta$ for some fixed $\eta>0$ (otherwise (4.20) is trivial). In all our algorithms, we may choose $\eta$ small enough to have $\mathbb{P}\left(\left|\rho\left(x+A_{x}\right)\right| \in\right.$ $\left.\left[\eta h, \eta^{-1} h\right]\right) \geq \delta$ for all $x \in \Gamma$, for some constant $\delta>0$. Therefore,

$$
\begin{aligned}
\mathbb{E}\left[\mathbb{1}_{\left\{x+A_{x} \in \Omega_{\mathrm{ext}}\right\}} \mathbb{P}\left(L_{1}^{c} \mid x_{0}=x+A_{x}\right)\right] & \geq \mathbb{E}\left[\mathbb{1}_{\left\{\left|\rho\left(x+A_{x}\right)\right| \in\left[\eta h, \eta^{-1} h\right]\right\}} \mathbb{P}\left(L_{1}^{c} \mid x_{0}=x+A_{x}\right)\right] \\
& \geq \frac{r \delta}{\sinh \left(r \bar{\kappa} / \sqrt{\varepsilon_{\mathrm{ext}}}\right)}\left(\frac{\sinh \left(r \bar{\kappa} / \sqrt{\varepsilon_{\mathrm{ext}}}\right)}{r}-\frac{\sinh \left(\left(r-\eta^{-1} h\right) \bar{\kappa} / \sqrt{\varepsilon_{\mathrm{ext}}}\right)}{\left(r-\eta^{-1} h\right)}\right) \geq C h
\end{aligned}
$$

for some constant $C>0$ independent of $h$ and $\varepsilon$. Combining this with the previous estimates ends the proof of Theorem 4.7 .

\section{NUMERICAL COMPARISONS}

The aim of this section is to compare the performance of the different ways to move the simulated Brownian particle after it hits the interface $\Gamma$ in the simple cases of a molecule constituted of one sphere and a molecule constituted of two spheres.

We compare the five different methods previously described in Sections 3.2.3, 3.3 and 4.1.2. These methods have two main characteristics: the jump direction and the jump asymmetry coefficient $\alpha$ (cf. Sect. 3.3).

- In four of them, the SNJ, ANJ, OANJ and UANJ algorithms, the particle only jumps in the normal direction to the interface. In the NTJ algorithm, the direction of replacement is chosen uniformly.

- The SNJ algorithm corresponds to the symmetric situation $\alpha=1$. In the ANJ and UANJ algorithms, $\alpha$ is a parameter. We focus on two values of $\alpha$. The first one, $\alpha=\varepsilon_{\text {ext }} / \varepsilon_{\text {int }}$, which corresponds to the value 19.875 in the numerical tests below, is chosen because it corresponds to a probability $\frac{1}{2}$ to go outside 
or inside the molecule. This enables us to compare the ANJ method with the NTJ method, which has the same asymmetry coefficient (see 3.16). The second value, $\alpha=10$, is intermediate between $\alpha=19.875$ and the symmetric case $\alpha=1$. Finally, the OANJ algorithm involves an asymmetry coefficient $\alpha^{*}$, optimal w.r.t. the bound (4.18). This coefficient is estimated with the Newton method.

We choose the same physical parameters as in [23]:

$$
\varepsilon_{\text {ext }}=78.5, \quad \varepsilon_{\text {int }}=4 \text { and } \bar{\kappa}=9.214 \times 10^{j}, \text { for } j=-1,0,1 .
$$

We also consider the case $\bar{\kappa}=9.214 \times 10^{-3}$ since the situation where $\bar{\kappa}$ is close to zero is relevant in biology. In such a situation, the end of the WOS algorithm is related to the probability for the Brownian motion to go to infinity.

We estimate the electrostatic free energy of the molecule (proportional to)

$$
\sum_{i=1}^{N} q_{i}\left(u\left(x_{i}\right)-u_{0}\left(x_{i}\right)\right),
$$

where the $x_{i}$ are the centers of the atoms of the molecule, with the following set of numerical parameters:

$$
\varepsilon=10^{-8}, \quad h \text { between } 0.3 \text { and } 10^{-4}, \text { and } 10^{6} \text { simulated paths. }
$$

The value of $\varepsilon$ is small enough to ensure that the error term $\varepsilon / h$ of the error estimates of the previous section has never an order larger than $h$. Note that choosing a very small absorption parameter $\varepsilon$ for the WOS outside $\Omega_{\text {int }}$ does not increase too much the CPU times as the corresponding mean computational cost is $O(|\log (\varepsilon)|)$.

Note that an interesting feature of these algorithms is that they only require a small amount of $\mathrm{RAM}^{5}$ and that they can be easily replicated on clustered processors. However, to make comparison easier, we run our $\mathrm{C} / \mathrm{C}++$ code sequentially on a single processor ${ }^{6}$.

\subsection{Numerical results on the case of a single sphere}

In the single sphere case, we fix

$$
\Omega_{\mathrm{int}}=B(0,1), \quad q=1, \quad x_{1}=(0,0,0) .
$$

The explicit solution given in (4.1) allows us to compare the numerical error with the theoretical bias computed in Section 4.1.

We first plot in Figure 4a the global CPU times (in seconds) as a function of $h$ in a logarithmic scale (base 10) in the case $\bar{\kappa}=9.214 \times 10^{1}$. Note that all the numerical tests have been done on the same computer ${ }^{6}$. We observe the same behaviour for all algorithms: the CPU time depend linearly of $h$, which is consistent with Corollary 4.6. Note that for any values of $h$ and $\bar{\kappa}$, a certain amount of CPU time is spent in the UWOS algorithm before the first exit of the particle. This explains the almost constant CPU time for large $h$ since, in this case, the particle is killed with a large probability immediately after its first exit (at least if $\bar{\kappa}$ is large enough). As expected, we observe also (except when $h$ is large) that the SNJ algorithm always requires a larger $\mathrm{CPU}$ time than the other algorithms.

Figure $4 \mathrm{~b}$ shows the CPU times as a function of $h$ for various values of $\bar{\kappa}$. We only plot the results for the NTJ algorithm, but we observe similar results for the other algorithms. The CPU time increases when $\bar{\kappa}$ decreases because the process is less killed outside the sphere. We can notice that these CPU times are very close for $\bar{\kappa}=9.214 \times 10^{-1}$ and $\bar{\kappa}=9.214 \times 10^{-3}$ especially for small $h$. This is certainly due to the fact that for such values the probability to be killed is related to the probability for the Brownian motion to go to infinity.

\footnotetext{
${ }^{5}$ Random Access Memory.

${ }^{6} \mathrm{CPU}$ E6850, $3.00 \mathrm{GHz}, \mathrm{i} 686$.
} 


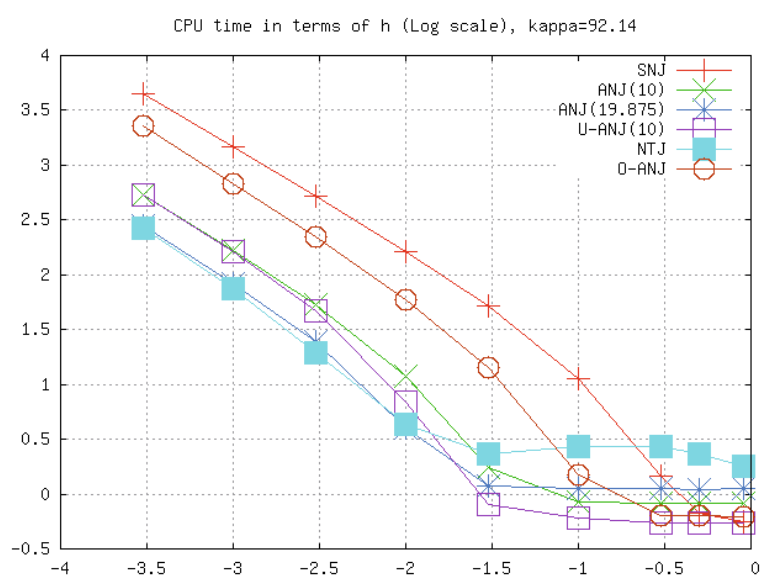

(a) CPU times (Log scale) in terms of $h$ (Log scale) for all the algorithms, $\bar{\kappa}=92.14$.

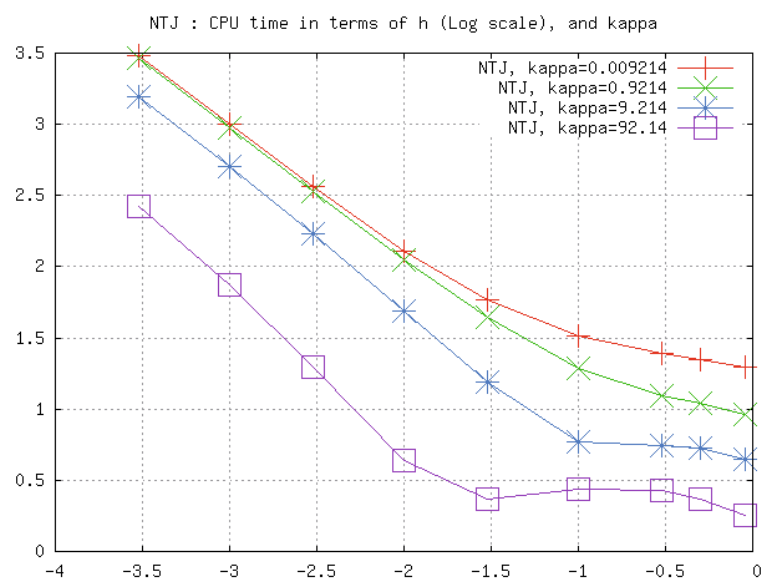

(b) CPU times (Log scale) in terms of $h$ (Log scale) for the NTJ algorithm, $\bar{\kappa}=9.214 \times 10^{j}$ for $j=-3,-1,0,1$.

Figure 4. Single sphere. CPU times.

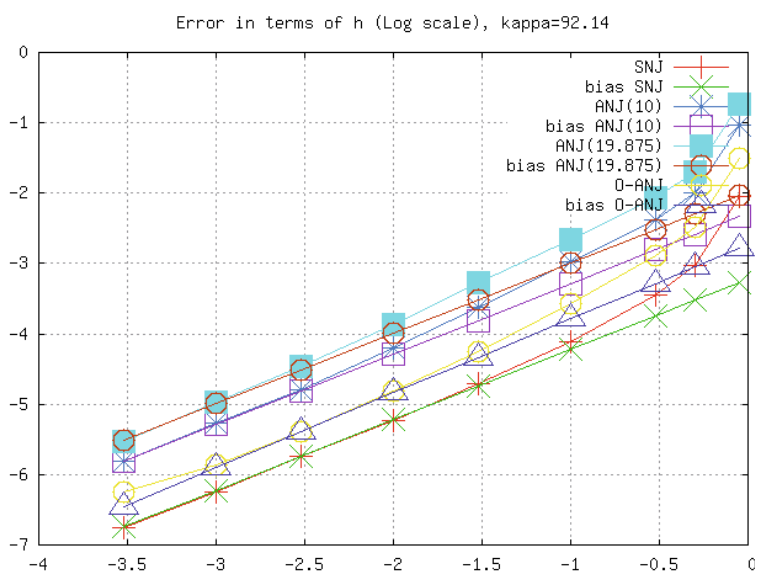

(a) The $*$ NJ algorithms, with $\bar{\kappa}=9.214 \times 10^{1}$.

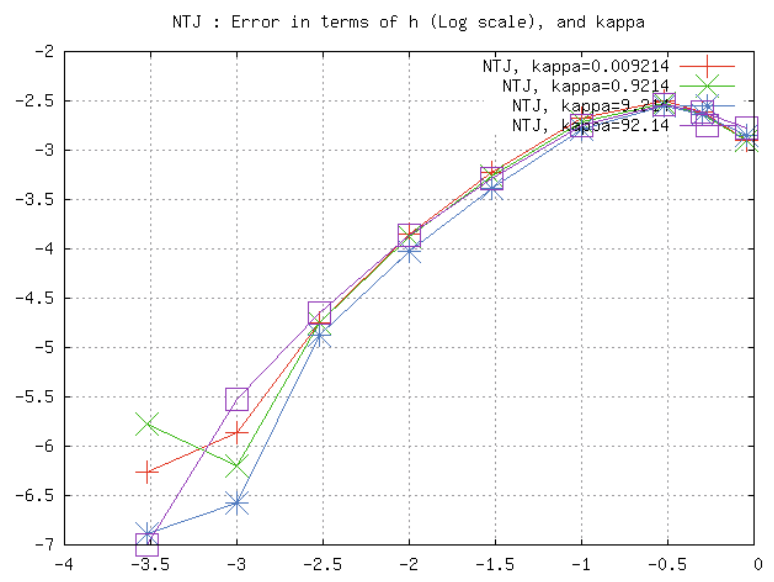

(b) The NTJ algorithm, $\bar{\kappa}=9.214 \times 10^{n}$ for $n=$ $-3,-1,0,1$.

Figure 5. Single sphere. Numerical error approximations (and their theoretical bias for the -NJ algorithm), in terms of $h$.

This is a good point for all our methods as, for even smaller physical values of $\bar{\kappa}$, the CPU time should not increase too much. Note that the theoretical bounds in Section 4.1.2 may not be optimal w.r.t. $\bar{\kappa}$.

Figure 5a shows the observed and theoretical bias of methods SNJ, OANJ and ANJ with $\alpha=10$ and $\alpha=19.875$, in the case $\bar{\kappa}=9.214 \times 10^{1}$. For all the methods, the theoretical and numerical bias agree and are of order one in $h$ as the slope of the function in logarithmic scale is one. We observe that the bias of the SNJ method is smaller than the other "normal jump" algorithms, except of course for the UANJ algorithm which is essentially unbiased (see Cor. 4.3). The corresponding curve is displayed in Figure 7 and only shows the Monte Carlo noise in the UANJ error. Note also that the methods OANJ and ANJ with $\alpha=19.875$ have almost the same bias when $h$ decreases. Other values of $\bar{\kappa}$ lead to similar conclusions. Concerning the NTJ algorithm, numerical results are shown in Figure $5 \mathrm{~b}$ for different values of $\bar{\kappa}$. In contrast with the normal jump methods, the slope of the curve in logarithmic scale is roughly 2 , which confirms the theoretical computations 


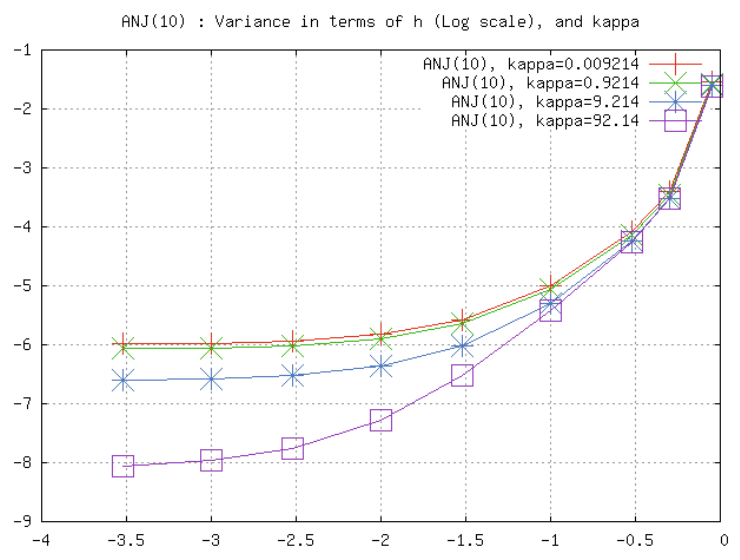

Figure 6. Single sphere. The (Log) variance in terms of $(\log ) h$.

of Corollary 4.3. For values of $h$ smaller than 0.001 , it usually provides at least one exact digit more than the three methods of Figure 5 a.

We have also computed the variance of the score for each method. Figure 6 shows the case of the ANJ algorithm with $\alpha=10$. We observe that the variance is very small and slowly decreases with $h$ and $\bar{\kappa}$. The variance has about the same behaviour for all the other methods.

Finally in Figure 7, we study the performances of the different methods. For each algorithm, we plot the error as a function of the CPU time. Such plots allow one to compare different methods at given CPU times. Not surprisingly, the UANJ method is the best one over the whole range of CPU times, as it is unbiased (in the case of a single sphere only). The irregular pattern of the corresponding curves comes from the Monte Carlo noise. This plot gives a good indicator of the Monte Carlo part of the global error for all the methods, as they all have comparable variances. The SNJ method is the worst one, especially when $\bar{\kappa}$ is small. The ANJ methods for $\alpha=10$ and $\alpha=19.875$ and the OANJ method have quite similar performances. As suggested by Figure 5b, the NTJ method is always more efficient when $h$ is small enough in all the examples studied: it is faster and its bias is of order two.

\subsection{The case of two spheres}

In this section we present numerical tests on a case with two spheres, parameterized by

$$
\Omega_{\mathrm{int}}=B\left(x_{1}, 1\right) \cup B\left(x_{2}, 1\right), \quad x_{1}=(0,0,0), \quad x_{2}=(0,0,1), \quad q_{1}=q_{2}=1 .
$$

The values of $\varepsilon$ and $h$ and the number of simulated paths are the same as in the case of a single sphere. The exact value of the free energy is unknown in this case.

Figure 8 shows the free energy approximations in terms of $h$ for the SNJ, ANJ(10), ANJ(19.875), UANJ(10), UANJ(19.875), and NTJ algorithms. We plot the free energy values for $\bar{\kappa}=9.214 \times 10^{-1}$ and $\bar{\kappa}=9.214 \times 10^{1}$. In these two cases, one can observe convergence of all the methods as $h$ decreases. Note that, for the ANJ $(\alpha)$ and NTJ algorithms, the convergence of the estimated free energies is not monotone w.r.t. $h$.

We also compute the free energy with the SNJ algorithm for $h=0.0001$, allowing us to estimate a pseudoerror w.r.t. this reference value for each algorithm. Figure 9 shows the resulting pseudo-error and performance (CPU time in terms of $h$ ) for $\bar{\kappa}=9.214$.

In Figure 9, we first observe that the error curves corresponding to the $\operatorname{ANJ}(\alpha)$ and NTJ algorithms all have a local minimum around $h=0.03$. This is due to the fact that the convergence of the estimated free energies is not monotone w.r.t. $h$. The expected bias for these methods, as a function of $h$, probably cuts the horizontal line corresponding to the exact free energy for a value of $h$ close to 0.03. Of course, these local minima have 


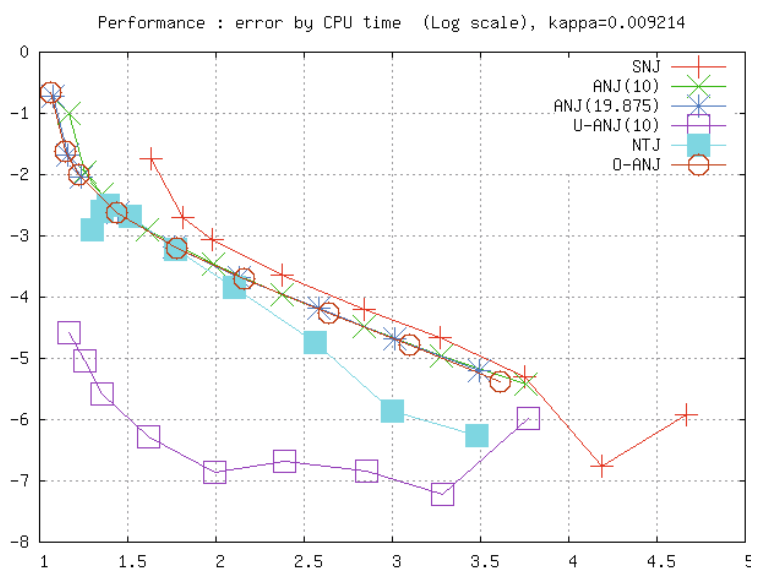

(a) $\bar{\kappa}=9.214 \times 10^{-3}$.

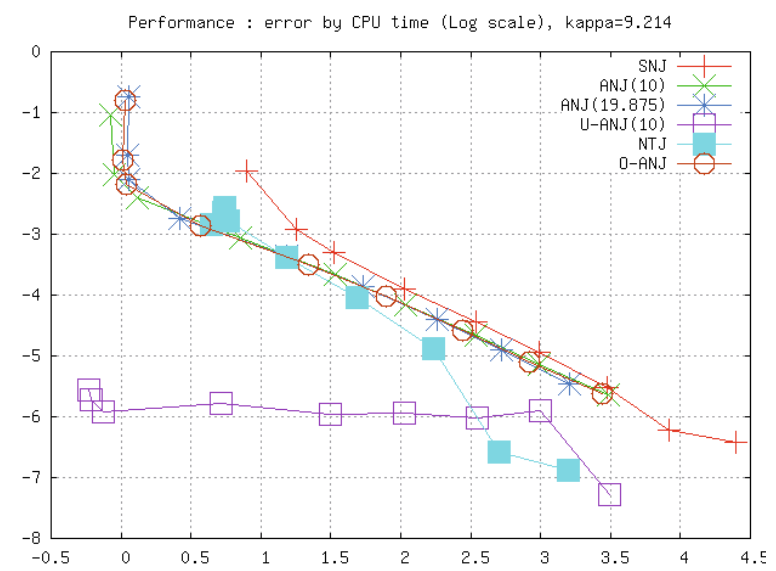

(c) $\bar{\kappa}=9.214$

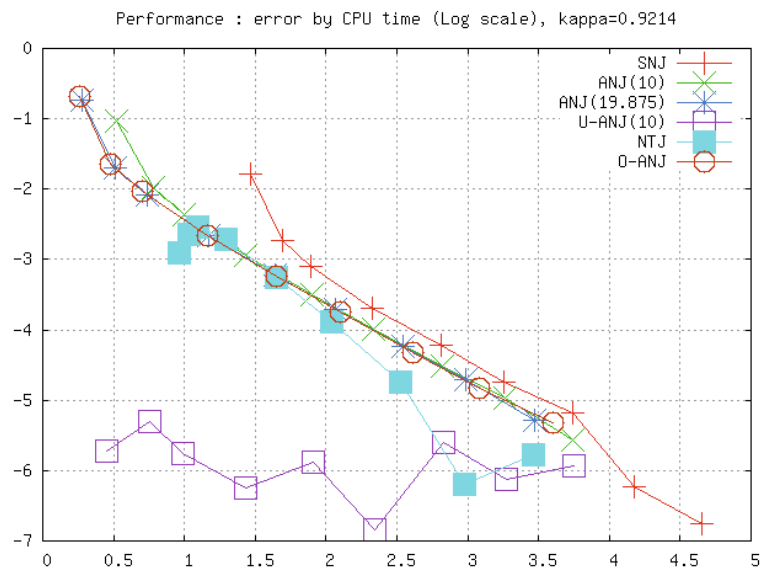

(b) $\bar{\kappa}=9.214 \times 10^{-1}$.

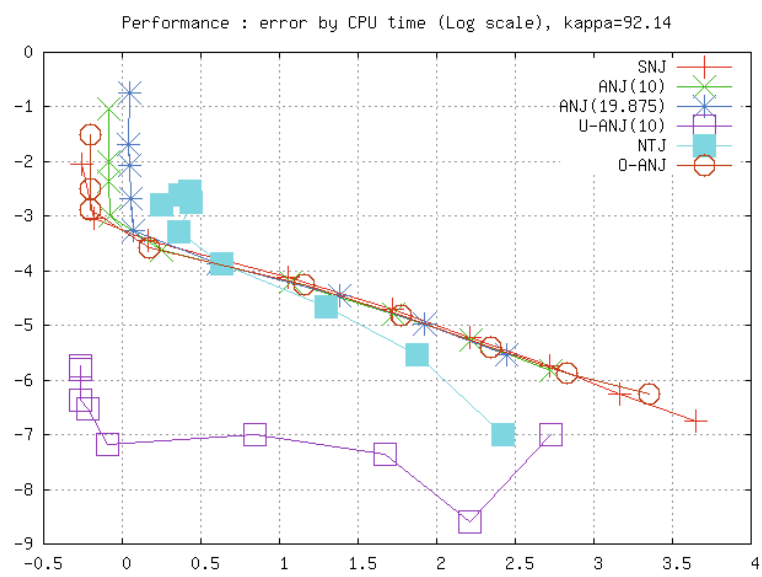

(d) $\bar{\kappa}=9.214 \times 10^{1}$.

Figure 7. Single sphere. Performance analysis.

no meaning in terms of performance. One should rather compare the parts of the curves corresponding to smaller $h$.

The SNJ method has a slightly smaller error than the other normal jump methods, but its performance is really worse. This is consistent with the case of a single sphere. The other normal jump methods $(\operatorname{ANJ}(\alpha)$ and $\operatorname{UANJ}(\alpha)$ ) have more or less the same behaviour both in terms of error and performance. This is in contrast with the case of a single sphere: obviously, the $\operatorname{UANJ}(\alpha)$ algorithms being designed for the one sphere problem only, they have no reason to be unbiased in the present situation. As in the case of a single sphere, the NTJ method outperforms all the previous ones. We expect that this conclusion is also valid for more realistic problems. As a conclusion, we recommend the NTJ method for more complex applications.

\section{A. Proof of Theorem 2.17}

Under Assumptions (H1)-(H4), the existence and uniqueness of a solution $u \in H^{1}(D)$ and the fact that $u \in C^{0}(\bar{D})$ are ensured by Theorems 8.3 and 8.30 of [9].

The first step of the proof consists in showing that $u$ restricted to $\Gamma \cap D$ is a $C^{\infty}$ function. To this aim, we consider of $C^{\infty}$ local straightenings of $\Gamma, \psi_{1}, \ldots, \psi_{M}$, defined on $\mathcal{U}_{1}, \ldots, \mathcal{U}_{M}$ respectively, constructed as 


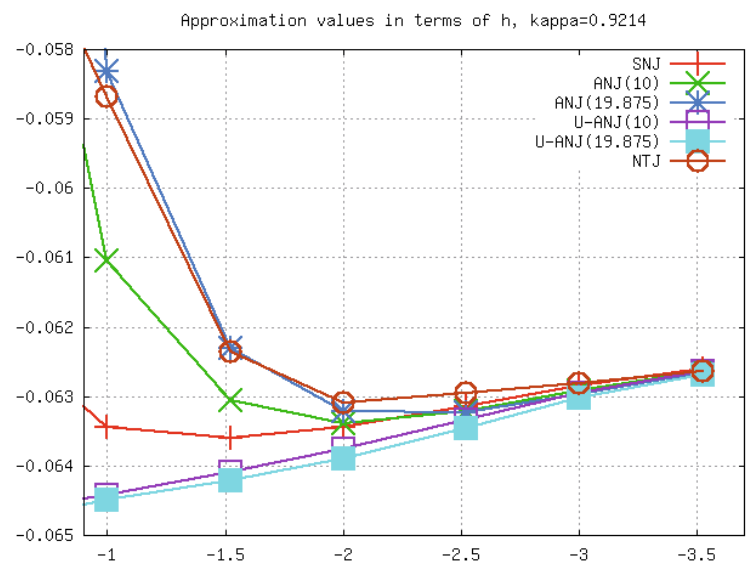

(a) $\bar{\kappa}=9.214 \times 10^{-1}$.

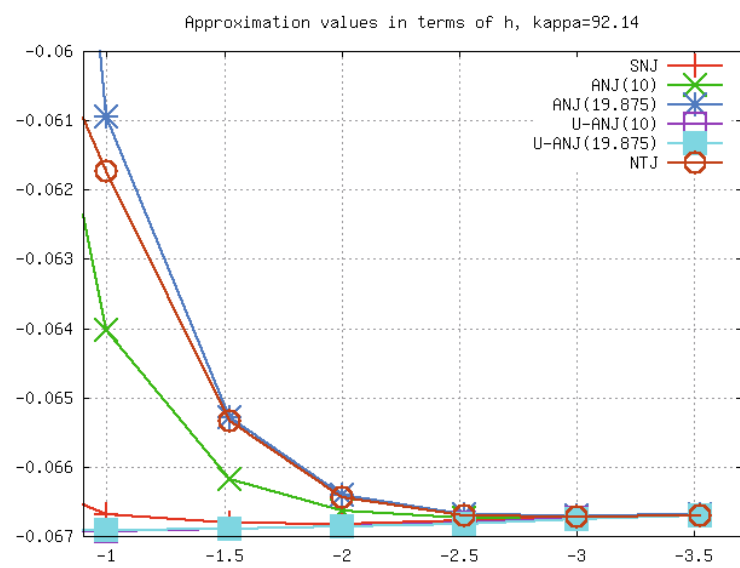

(b) $\bar{\kappa}=9.214 \times 10^{1}$.

Figure 8. Two spheres case. The free energy approximations, in terms of $h(\log )$.

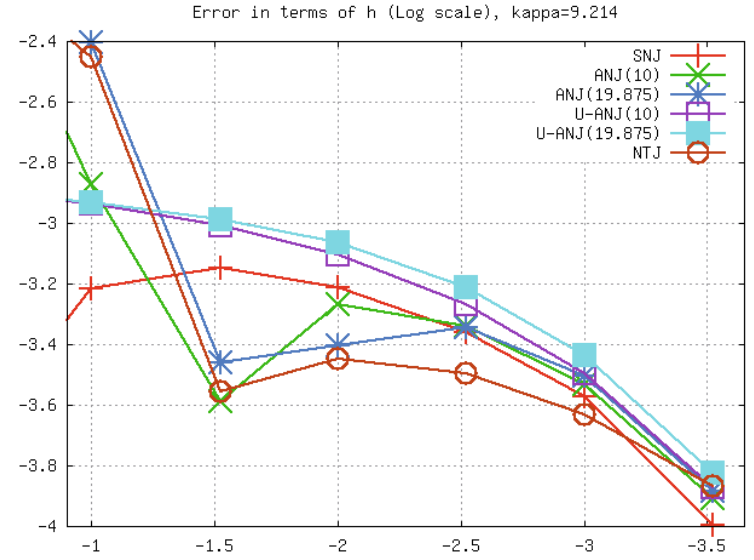

(a) Pseudo error. $\bar{\kappa}=9.214$.

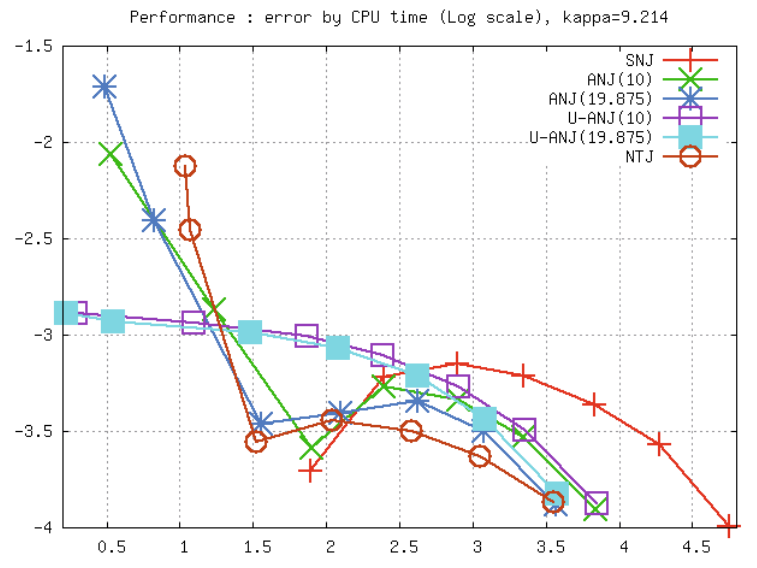

(b) Performance measure. $\bar{\kappa}=9.214$

Figure 9. Two spheres case. Pseudo error ( $\log )$, and performance measure for the free energy approximation in terms of $h(\mathrm{Log})$.

in Proposition 2.1. Replacing $\mathcal{U}_{i}$ by $\mathcal{U}_{i} \cap D$, we may assume that $\mathcal{U}_{m} \subset D$. Let us fix $i \in\{1, \ldots, M\}$ and write for simplicity $\psi=\psi_{i}, \mathcal{U}=\mathcal{U}_{i}$ and $\mathcal{V}=\psi(\mathcal{U})$.

We define $v(y)=u\left(\psi^{-1}(y)\right), \tilde{\varepsilon}(y)=\varepsilon\left(\psi^{-1}(y)\right), \tilde{g}(y)=g\left(\psi^{-1}(y)\right), \tilde{\lambda}(y)=\lambda\left(\psi^{-1}(y)\right)$ and $M(y)=$ $\left(M_{i j}(y)\right)_{1 \leq i, j \leq d}=J \psi\left(\psi^{-1}(y)\right)$ for all $y \in \mathcal{V}$. Note that, since $\psi$ is $C^{\infty}$ diffeomorphism from $\mathcal{U}$ to $\mathcal{V}$, we have $v \in \bar{H}_{\text {loc }}^{1}(\mathcal{V})$.

With these notations, the PDE (2.42) may be rewritten on $\mathcal{U}$ as

$$
-\nabla \cdot[\tilde{\varepsilon}(\psi(x)) M(\psi(x)) \nabla v(\psi(x))]+\tilde{\lambda}(\psi(x)) v(\psi(x))=\tilde{g}(\psi(x))
$$

and

$$
\nabla \cdot[\tilde{\varepsilon}(\psi(x)) M(\psi(x)) \nabla v(\psi(x))]=\sum_{i=1}^{d} \nabla\left[\tilde{\varepsilon} \sum_{j=1}^{d} M_{i j} \partial_{j} v\right](\psi(x)) \partial_{i} \psi(x)=\sum_{i, j, k=1}^{d} \partial_{k}\left(\tilde{\varepsilon} M_{i j} \partial_{j} v\right)(\psi(x)) M_{i k}(\psi(x)),
$$


where we write in this section $\partial_{i}$ for the partial derivative w.r.t. the $i$ th variable. Therefore, the change of variable $y=\psi(x)$ leads to the $\mathrm{PDE}^{7}$

$$
-\sum_{i, j, k=1}^{d} M_{i k} \partial_{k}\left(\tilde{\varepsilon} M_{i j} \partial_{j} v\right)+\tilde{\lambda} v=\tilde{g} \quad \text { on } \mathcal{V} .
$$

We are going to use the next lemma repeatedly in the proof. Its proof is postponed at the end of this section.

Lemma A.1. For all $i \in\{1, \ldots, d\}$, on $\mathcal{V}$,

$$
\sum_{k=1}^{d} \partial_{k}\left(M_{i k} \operatorname{det} J \psi^{-1}\right)=0
$$

Step 0. To start with, we recall how classical energy computations can be used to prove that $\nabla v \in L_{\text {loc }}^{2}(\mathcal{V})$ from the fact that $v \in L_{\text {loc }}^{2}(\mathcal{V})$. In the next steps of the proof, we will extend this method to prove $L^{2}$ estimates for higher-order derivatives of $v$ (in the sense of distributions).

Let $\chi \in C_{c}^{\infty}(\mathcal{V})$ and set $w=\chi v$. It follows from elementary computations that

$$
-\sum_{i, j, k=1}^{d} M_{i k} \partial_{k}\left(\tilde{\varepsilon} M_{i j} \partial_{j} w\right)+\sum_{i, j, k=1}^{d} M_{i k} \partial_{k}\left(\tilde{\varepsilon} M_{i j} v \partial_{j} \chi\right)+\tilde{\lambda} w=\chi \tilde{g}-\sum_{i, j, k=1}^{\mathrm{d}} \tilde{\varepsilon} M_{i j} M_{i k} \partial_{k} \chi \partial_{j} v .
$$

Multiplying this equation by $w \operatorname{det} J \psi^{-1}$, integrating over $\mathcal{V}$, integrating by parts the first and second terms of the left-hand side, and using Lemma A.1, we get

$$
\begin{aligned}
& \int_{\mathcal{V}} \tilde{\varepsilon}(y)|M(y) \nabla w(y)|^{2} \operatorname{det} J \psi^{-1}(y) \mathrm{d} y-\int_{\mathcal{V}} \tilde{\varepsilon}(y) v(y) \operatorname{det} J \psi^{-1}(y) \nabla \chi(y)^{T} M(y)^{T} M(y) \nabla w(y) \mathrm{d} y \\
& +\int_{\mathcal{V}} \tilde{\lambda}(y) w^{2}(y) \operatorname{det} J \psi^{-1}(y) \mathrm{d} y=\int_{\mathcal{V}_{m}} \chi(y) \tilde{g}(y) w(y) \operatorname{det} J \psi^{-1}(y) \mathrm{d} y \\
& -\sum_{i, j, k=1}^{d} \int_{\mathcal{V}} \tilde{\varepsilon}(y) M_{i j}(y) M_{i k}(y) \operatorname{det} J \psi^{-1}(y) \partial_{k} \chi(y) v(y)\left(\partial_{j} w(y)-v(y) \partial_{j} \chi(y)\right) \mathrm{d} y,
\end{aligned}
$$

where we have used in the last term the relation

$$
w(y) \partial_{j} v(y)=v(y)\left(\partial_{j} w(y)-v(y) \partial_{j} \chi(y)\right) .
$$

Using the fact that $\tilde{\varepsilon}, \tilde{\lambda}, \tilde{g}, M, \chi, \nabla \chi$ and $\operatorname{det} J \psi^{-1}$ are bounded on the support of $\chi$, and that $\tilde{\varepsilon}$ and $\operatorname{det} J \psi^{-1}$ are bounded away from 0 on this set, we obtain

$$
\int_{\mathcal{V}}|M(y) \nabla w(y)|^{2} \mathrm{~d} y \leq C \int_{\operatorname{Supp}(\chi)}\left(|w(y)|+|w(y)|^{2}+|v(y)||\nabla w(y)|+|v(y)|^{2}\right) \mathrm{d} y
$$

for some constant $C$.

Now, letting $S^{d-1}$ be the two-dimensional unit sphere in $\mathbb{R}^{d}$, the function $(s, y) \mapsto|M(y) s|^{2}$ is positive and continuous on the compact set $S^{d-1} \times \operatorname{Supp}(\chi)$. Therefore,

$$
C^{\prime}:=\inf _{(s, y) \in S^{d-1} \times \operatorname{Supp}(\chi)}|M(y) s|^{2}>0 .
$$

\footnotetext{
${ }^{7}$ Of course, since $v$ is only $H^{1}$ and $\tilde{\varepsilon}$ is discontinuous, all this computation and the PDE (A.1) should be understood in the weak sense.
} 
Hence, using Young's inequality

$$
2 a b \leq \eta^{-1} a^{2}+\eta b^{2}
$$

with $a=|v(y)|, b=|\nabla w(y)|$ and $0<\eta<1 /\left(4 C C^{\prime}\right)$, we obtain that

$$
\|\nabla w\|_{L^{2}(\mathcal{V})}^{2} \leq C^{\prime \prime}\left(\|w\|_{L^{2}(\operatorname{Supp}(\chi))}^{2}+\|v\|_{L^{2}(\operatorname{Supp}(\chi))}^{2}\right)<+\infty
$$

for some constant $C^{\prime \prime}$. Since this holds for all $\chi \in C_{c}^{\infty}(\mathcal{V})$, we finally obtain that $\nabla v \in L_{\text {loc }}^{2}(\mathcal{V})$.

Step 1. We now adapt the previous computation to prove that $\nabla\left(\partial_{2} v\right) \in L_{\text {loc }}^{2}(\mathcal{V})$. First, we compute the partial derivative of equation (A.1) w.r.t. the second coordinate of $y \in \mathcal{V}$, using the fact that $\tilde{\varepsilon}$ only depends of the first coordinate:

$$
-\sum_{i, j, k=1}^{d} M_{i k} \partial_{k}\left(\tilde{\varepsilon} M_{i j} \partial_{2, j} v\right)+\tilde{\lambda} \partial_{2} v+\partial_{2} \tilde{\lambda} v=\partial_{2} \tilde{g}+\sum_{i, j, k=1}^{d} M_{i k} \partial_{k}\left(\tilde{\varepsilon} \partial_{2} M_{i j} \partial_{j} v\right)+\sum_{i, j, k=1}^{d} \partial_{2} M_{i k} \partial_{k}\left(\tilde{\varepsilon} M_{i j} \partial_{j} v\right)
$$

We fix $\chi \in C_{c}^{\infty}(\mathcal{U})$ and we define $w=\chi \partial_{2} v$. Then

$$
\begin{array}{r}
-\sum_{i, j, k=1}^{d} M_{i k} \partial_{k}\left(\tilde{\varepsilon} M_{i j} \partial_{j} w\right)+\tilde{\lambda} w+\chi v \partial_{2} \tilde{\lambda}=\chi \partial_{2} \tilde{g}+\sum_{i, j, k=1}^{d} \chi M_{i k} \partial_{k}\left(\tilde{\varepsilon} \partial_{2} M_{i j} \partial_{j} v\right)+\sum_{i, j, k=1}^{d} \chi \partial_{2} M_{i k} \partial_{k}\left(\tilde{\varepsilon} M_{i j} \partial_{j} v\right) \\
-\sum_{i, j, k=1}^{d} M_{i k} \partial_{k}\left(\tilde{\varepsilon} M_{i j} \partial_{j} \chi \partial_{2} v\right)-\sum_{i, j, k=1}^{d} \tilde{\varepsilon} M_{i j} M_{i k} \partial_{k} \chi \partial_{2, j} v . \quad(\mathrm{A} .
\end{array}
$$

Multiplying the previous equation by $w \operatorname{det} J \psi^{-1}$, integrating over $\mathcal{V}$, integrating by parts every term involving a partial derivative of $\tilde{\varepsilon}$ and using Lemma A.1 when necessary, we obtain

$$
\begin{aligned}
\int_{\mathcal{V}}|M(y) \nabla w(y)|^{2} \mathrm{~d} y \leq C \int_{\operatorname{Supp}(\chi)}\left(|w(y)|+|w(y)|^{2}+|w(y) v(y)|\right. & \left.+(|w(y)|+|\nabla w(y)|)|\nabla v(y)|+\left|w(y) \nabla \partial_{2} v(y)\right|\right) \mathrm{d} y
\end{aligned}
$$

Using (A.2), the relation $w(y) \nabla \partial_{2} v(y)=\partial_{2} v(y)\left(\nabla w(y)-\nabla \chi(y) \partial_{2} v(y)\right)$, and the inequality (A.3) with an appropriate $\eta>0$ to bound $|\nabla w(y)||\nabla v(y)|$, we finally obtain that

$$
\|\nabla w\|_{L^{2}(\mathcal{V})} \leq C\left(\|w\|_{L^{2}(\operatorname{Supp}(\chi))}+\|v\|_{L^{2}(\operatorname{Supp}(\chi))}+\|\nabla v\|_{L^{2}(\operatorname{Supp}(\chi))}\right)<+\infty
$$

and hence $\nabla\left(\partial_{2} v\right) \in L_{\text {loc }}^{2}(\mathcal{V})$. 
Step 2. Following the same method, we can now prove by induction over $n$ that for all $n \geq 0$ and $k_{1}, \ldots, k_{n} \in$ $\{2, \ldots, d\}, \nabla\left(\partial_{k_{1}, \ldots, k_{n}} v\right) \in L_{\text {loc }}^{2}(\mathcal{V})$. Assume that this holds for $n-1 \geq 0$, fix $k_{1}, \ldots, k_{n} \in\{2, \ldots, d\}$, fix $\chi \in C_{c}^{\infty}(\mathcal{V})$ and define $w=\chi \partial_{k_{1}, \ldots, k_{n}} v$.

Considering the $n$th order partial derivative of (A.1) with respect to $y_{k_{1}}, \ldots, y_{k_{n}}$, writing the PDE solved by $w$ as in (A.4), multiplying the result by $w \operatorname{det} J \psi^{-1}$, integrating over $\mathcal{V}$ and integrating by parts each term involving a partial derivative of $\tilde{\varepsilon}$, we obtain as above

$$
\begin{aligned}
\int_{\mathcal{V}}|M(y) \nabla w(y)|^{2} \mathrm{~d} y \leq & C \int_{\operatorname{Supp}(\chi)}\left(|w(y)|+|w(y)|^{2}+|w(y)|\left(\sum_{j=0}^{n} \sum_{1 \leq i_{1}<\ldots<i_{j} \leq n}\left|\partial_{k_{i_{1}}, \ldots, k_{i_{j}}} v(y)\right|\right)\right. \\
& \left.+(|w(y)|+|\nabla w(y)|)\left(\sum_{j=0}^{n-1} \sum_{1 \leq i_{1}<\ldots<i_{j} \leq n}\left|\nabla \partial_{k_{i_{1}}, \ldots, k_{i_{j}}} v(y)\right|\right)+\left|w(y) \nabla \partial_{k_{1}, \ldots, k_{n}} v(y)\right|\right) \mathrm{d} y .
\end{aligned}
$$

The key point is that $j \leq n-1$ in the second sum, so that only $n$th order derivatives of $v$ are involved, for which $L^{2}$ bounds are known by assumption. Therefore, using the relation

$$
w(y) \nabla \partial_{k_{1}, \ldots, k_{n}} v(y)=\partial_{k_{1}, \ldots, k_{n}} v(y)\left(\nabla w(y)-\nabla \chi(y) \partial_{k_{1}, \ldots, k_{n}} v(y)\right)
$$

and applying (A.3) with appropriate constants, one has

$$
\|\nabla w\|_{L^{2}(\mathcal{V})}^{2} \leq C\left(\|w\|_{L^{2}(\operatorname{Supp}(\chi))}^{2}+\|v\|_{L^{2}(\operatorname{Supp}(\chi))}^{2}+\sum_{j=0}^{n-1} \sum_{i_{1}, \ldots, i_{j} \in\{2, \ldots, d\}}\left\|\nabla \partial_{i_{1}, \ldots, i_{j}} v\right\|_{L^{2}(\operatorname{Supp}(\chi))}^{2}\right)<+\infty .
$$

Hence $\nabla\left(\partial_{k_{1}, \ldots, k_{n}} v\right) \in L_{\text {loc }}^{2}(\mathcal{V})$.

Step 3. Summarizing the previous results, we have $v \in H_{\mathrm{loc}}^{1, \infty, \ldots, \infty}(\mathcal{V})$, which means that $v$ and $\partial v / \partial x_{1}$ are $H^{\infty}$ w.r.t. the variables $x_{2}, \ldots, x_{d}$. By classical trace results (see e.g. [5]), we deduce that $v_{\mid\left\{y_{1}=0\right\}}$ admits, as a function of $\left(y_{2}, \ldots, y_{d}\right) \in \psi(\mathcal{U} \cap \Gamma)$, partial derivatives of any order in $L_{\text {loc }}^{2}(\psi(\mathcal{U} \cap \Gamma))$. Therefore, $v_{\mid\left\{y_{1}=0\right\}} \in$ $C^{\infty}(\psi(\mathcal{U} \cap \Gamma))$. Since $\psi$ and $\Gamma$ are $C^{\infty}$, we finally obtain that $u_{\mid \Gamma} \in C^{\infty}(\Gamma \cap D)$.

Step 4. The problem (2.42) can be decomposed into the following two sub-problems: $u=u_{\text {int }}$ in $\overline{\Omega_{\text {int }}} \cap D$ and $u=u_{\text {ext }}$ in $\overline{\Omega_{\text {ext }}} \cap D$, where

$$
\begin{cases}-\varepsilon_{\text {int }} \Delta u_{\text {int }}(x)+\lambda(x) u_{\text {int }}(x)=g(x) & \text { for } x \in \Omega_{\text {int }} \cap D \\ u_{\text {int }}(x)=u_{\mid \Gamma}(x) & \text { for } x \in \Gamma \cap D \\ u_{\text {int }}(x)=h(x) & \text { for } x \in \Omega_{\text {int }} \cap \partial D\end{cases}
$$

and

$$
\begin{cases}-\varepsilon_{\mathrm{ext}} \Delta u_{\mathrm{ext}}(x)+\lambda(x) u_{\mathrm{ext}}(x)=g(x) & \text { for } x \in \Omega_{\mathrm{ext}} \cap D \\ u_{\mathrm{ext}}(x)=u_{\mid \Gamma}(x) & \text { for } x \in \Gamma \cap D \\ u_{\mathrm{ext}}(x)=h(x) & \text { for } x \in \Omega_{\mathrm{ext}} \cap \partial D .\end{cases}
$$

Regularity results are well-known for these Poisson equations: it follows from Theorem 6.13 of [9] that $u_{\text {ext }}$ is $C^{2, \alpha}$ (i.e. $C^{2}$ with $\alpha$-Hölder second order derivatives) on any compact subset of $D \cap \Omega_{\text {ext }}$, and next from Lemma 6.18 of [9] that $u_{\text {ext }} \in C^{2, \alpha}\left(D \cap \overline{\Omega_{\text {ext }}}\right)$; it also follows from the same results that $u_{\text {int }} \in C^{2}\left(D \cap \overline{\Omega_{\text {int }}}\right)$. From this, we deduce that $u \in C^{0}(\bar{D}) \cap C^{2}(D \backslash \Gamma)$. Since $\varepsilon_{\text {int }} \nabla u_{\text {int }}(y) \cdot n(y)=\varepsilon_{\text {ext }} \nabla u_{\text {ext }}(y) \cdot n(y)$ for all $y \in \Gamma \cap D$, it is also trivial to check that $\hat{u} \in C^{1}(D)$. Moreover, the fact that $\hat{u} \in W_{\mathrm{loc}}^{2, \infty}(D)$ is obvious. 
It now only remains to check that $u$ is bounded on $\bar{D}$. Since $g$ has compact support, $u$ is solution to $-\varepsilon_{\text {ext }} \Delta u+\lambda(x) u=0$ in $D$ outside of some (large) ball $B$. Since the function $u$ is bounded on $\partial(D \cap B)$, the boundedness of $u$ in $D \cap B$ follows from the maximum principle (see e.g. Thm. 3.5 in [9]).

Proof of Lemma A.1. Since the Fréchet derivative of the determinant function at the invertible matrix $A$ is the linear map $H \mapsto \operatorname{Tr}\left(H A^{-1}\right) \operatorname{det} A$, we only have to prove that

$$
\sum_{k=1}^{d} M_{i k}(y) \operatorname{Tr}\left(\partial_{k} J \psi^{-1}(y) J \psi(y)\right)=-\sum_{i=1}^{d} \partial_{k} M_{i k}(y)
$$

The left-hand side of the previous equation is equal to

$$
\sum_{k=1}^{d} M_{i k}(y) \sum_{j, l=1}^{d} \partial_{k} J \psi_{l j}^{-1}(y) M_{j l}(y)=\sum_{j, l=1}^{d} M_{j l}(y) \sum_{k=1}^{d} M_{i k}(y) \partial_{l} J \psi_{k j}^{-1}(y)
$$

since $\partial_{k} J \psi_{l j}^{-1}=\partial_{l} J \psi_{k j}^{-1}$, as symmetric second-order partial derivatives. Expliciting the $l$-th partial derivative of each term of the equality $M(y) J \psi^{-1}(y)=\mathrm{Id}$, we deduce that

$$
-\sum_{k, l=1}^{d} \partial_{l} M_{i k}(y) \sum_{j=1}^{d} J \psi_{k j}^{-1}(y) M_{j l}(y)=-\sum_{k=1}^{d} \partial_{k} M_{i k}(y)
$$

since $J \psi^{-1}(y) M(y)=$ Id. This ends the proof of Lemma A.1.

\section{REFERENCES}

[1] D.G. Aronson, Bounds for the fundamental solution of a parabolic equation. Bull. Amer. Math. Soc. 73 (1967) 890-896.

[2] N.A. Baker, D. Sept, M.J. Holst and J.A. McCammon, The adaptive multilevel finite element solution of the Poisson-Boltzmann equation on massively parallel computers. IBM J. Res. Dev. 45 (2001) 427-437.

[3] N.A. Baker, D. Bashford and D.A. Case, Implicit solvent electrostatics in biomolecular simulation, in New algorithms for macromolecular simulation, Lect. Notes Comput. Sci. Eng. 49, Springer, Berlin (2005) 263-295.

[4] A.N. Borodin and P. Salminen, Handbook of Brownian motion-facts and formulae. Probability and its Applications, 2nd edition, Birkhäuser Verlag, Basel (2002).

[5] H. Brezis, Analyse fonctionnelle : Théorie et applications. Collection Mathématiques Appliquées pour la Maîtrise, Masson, Paris (1983).

[6] R. Dautray and J.-L. Lions, Evolution problems II, Mathematical analysis and numerical methods for science and technology 6. Springer-Verlag, Berlin (1993)

[7] S.N. Ethier and T.G. Kurtz, Markov processes - Characterization and convergence. Wiley Series in Probability and Mathematical Statistics, Probability and Mathematical Statistics, John Wiley \& Sons Inc., New York (1986).

[8] M. Fukushima, Y. Ōshima and M. Takeda, Dirichlet forms and symmetric Markov processes, de Gruyter Studies in Mathematics 19. Walter de Gruyter \& Co., Berlin (1994).

[9] D. Gilbarg and N.S. Trudinger, Elliptic partial differential equations of second order. Classics in Mathematics, Reprint of the 1998 edition, Springer-Verlag, Berlin (2001).

[10] N. Ikeda and S. Watanabe, Stochastic differential equations and diffusion processes, North-Holland Mathematical Library 24. Second edition, North-Holland Publishing Co., Amsterdam (1989).

[11] I. Karatzas and S.E. Shreve, Brownian motion and stochastic calculus, Graduate Texts in Mathematics 113. Second edition, Springer-Verlag, New York (1991).

[12] O.A. Ladyzhenskaya and N.N. Ural'tseva, Linear and quasilinear elliptic equations. Academic Press, New York (1968).

[13] B. Lapeyre, É. Pardoux and R. Sentis, Introduction to Monte-Carlo methods for transport and diffusion equations, Oxford Texts in Applied and Engineering Mathematics 6. Oxford University Press, Oxford (2003).

[14] J.-F. Le Gall, One-dimensional stochastic differential equations involving the local times of the unknown process, in Stochastic analysis and applications (Swansea, 1983), Lecture Notes Math. 1095, Springer, Berlin (1984) 51-82.

[15] A. Lejay, Méthodes probabilistes pour l’homogénéisation des opérateurs sous forme divergence: Cas linéaires et semi-linéaires. Ph.D. Thesis, Université de Provence, Marseille, France (2000). 
[16] A. Lejay and S. Maire, Simulating diffusions with piecewise constant coefficients using a kinetic approximation. Comput. Meth. Appl. Mech. Eng. 199 (2010) 2014-2023.

[17] A. Lejay and M. Martinez, A scheme for simulating one-dimensional diffusion processes with discontinuous coefficients. Ann. Appl. Probab. 16 (2006) 107-139.

[18] N. Limić, Markov jump processes approximating a nonsymmetric generalized diffusion. Preprint, arXiv:0804.0848v4 (2008).

[19] S. Maire, Réduction de variance pour l'intégration numérique et pour le calcul critique en transport neutronique. Ph.D. Thesis, Université de Toulon et du Var, France (2001).

[20] S. Maire and D. Talay, On a Monte Carlo method for neutron transport criticality computations. IMA J. Numer. Anal. 26 (2006) 657-685.

[21] M. Martinez, Interprétations probabilistes d'opérateurs sous forme divergence et analyse des méthodes numériques probabilistes associées. Ph.D. Thesis, Université de Provence, Marseille, France (2004).

[22] M. Martinez and D. Talay, Discrétisation d'équations différentielles stochastiques unidimensionnelles à générateur sous forme divergence avec coefficient discontinu. C. R. Math. Acad. Sci. Paris 342 (2006) 51-56.

[23] M. Mascagni and N.A. Simonov, Monte Carlo methods for calculating some physical properties of large molecules. SIAM J. Sci. Comput. 26 (2004) 339-357.

[24] N.I. Portenko, Diffusion processes with a generalized drift coefficient. Teor. Veroyatnost. i Primenen. 24 (1979) 62-77.

[25] N.I. Portenko, Stochastic differential equations with a generalized drift vector. Teor. Veroyatnost. i Primenen. 24 (1979) 332-347.

[26] P.E. Protter, Stochastic integration and differential equations - Second edition, Version 2.1, Stochastic Modelling and Applied Probability 21. Corrected third printing, Springer-Verlag, Berlin (2005).

[27] D. Revuz and M. Yor, Continuous martingales and Brownian motion, Grundlehren der Mathematischen Wissenschaften 293. Springer-Verlag, Berlin (1991).

[28] L.C.G. Rogers and D. Williams, Foundations, Diffusions, Markov processes, and martingales 1. Reprint of the second edition (1994), Cambridge Mathematical Library, Cambridge University Press, Cambridge (2000).

[29] L.C.G. Rogers and D. Williams, Itô calculus, Diffusions, Markov processes, and martingales 2. Reprint of the second edition (1994), Cambridge Mathematical Library, Cambridge University Press, Cambridge (2000).

[30] A. Rozkosz and L. Słomiński, Extended convergence of Dirichlet processes. Stochastics Stochastics Rep. 65 (1998) 79-109.

[31] K.K. Sabelfeld, Monte Carlo methods in boundary value problems. Springer Series in Computational Physics, Springer-Verlag, Berlin (1991).

[32] K.K. Sabelfeld and D. Talay, Integral formulation of the boundary value problems and the method of random walk on spheres. Monte Carlo Meth. Appl. 1 (1995) 1-34.

[33] N.A. Simonov, Walk-on-spheres algorithm for solving boundary-value problems with continuity flux conditions, in Monte Carlo and quasi-Monte Carlo methods 2006, Springer, Berlin (2008) 633-643.

[34] N.A. Simonov, M. Mascagni and M.O. Fenley, Monte Carlo-based linear Poisson-Boltzmann approach makes accurate saltdependent solvation free energy predictions possible. J. Chem. Phys. 127 (2007) 185105.

[35] D.W. Stroock, Diffusion semigroups corresponding to uniformly elliptic divergence form operators, in Séminaire de Probabilités, XXII, Lecture Notes in Math. 1321, Springer, Berlin (1988) 316-347.

[36] D.W. Stroock and S.R.S. Varadhan, Multidimensional diffusion processes, Grundlehren der Mathematischen Wissenschaften 233. Springer-Verlag, Berlin (1979). 